
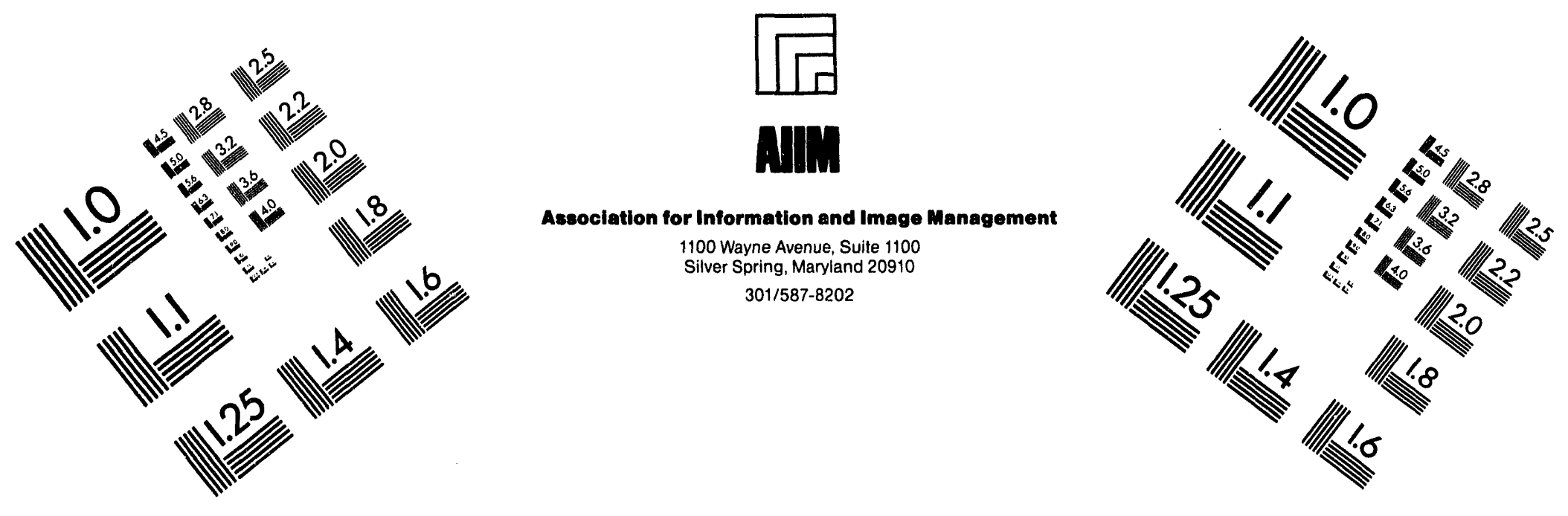

\title{
Centimeter
}

1 Inches
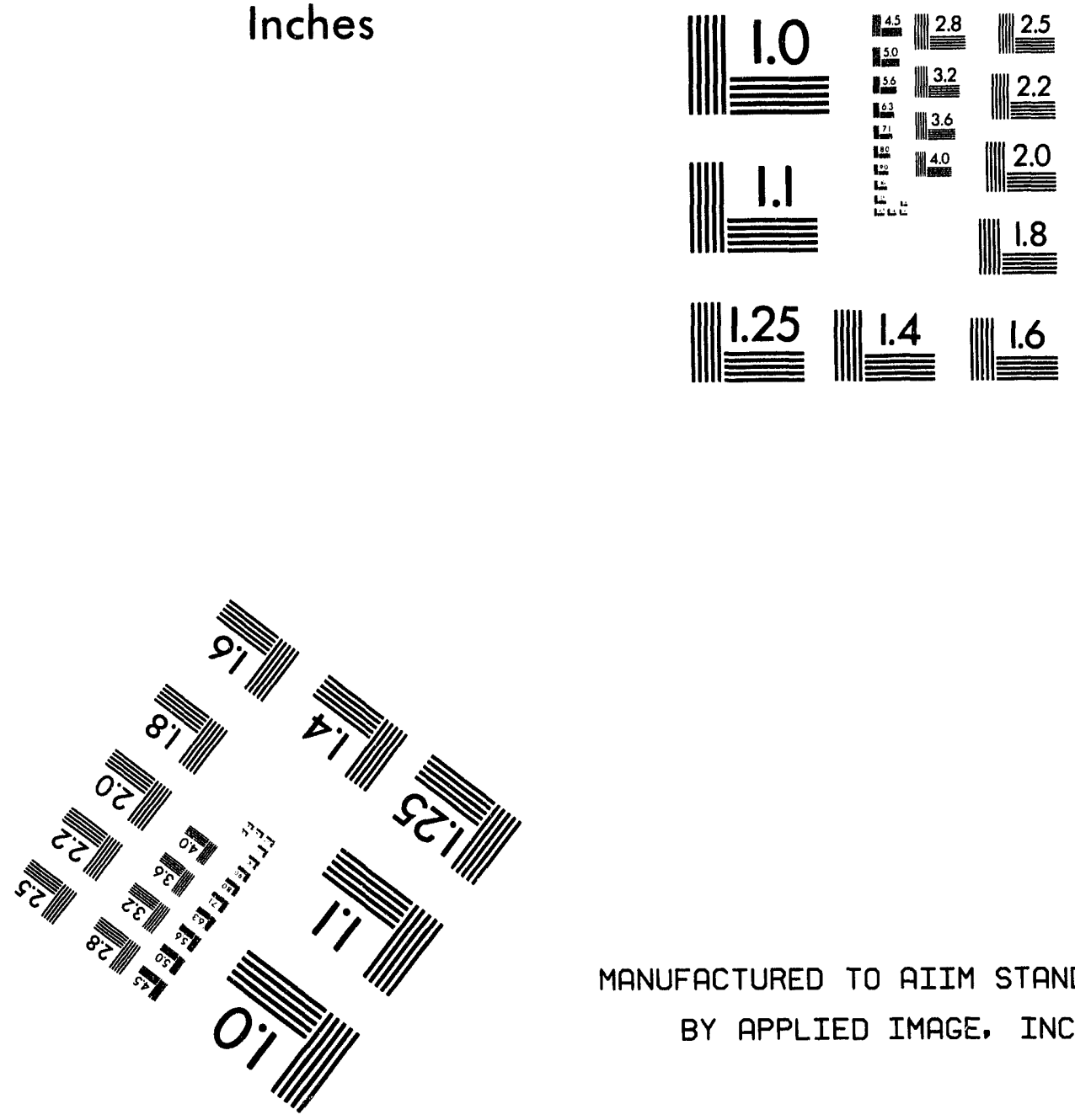

MANUFACTURED TO AIIM STANDARDS

BY APPLIED IMAGE, INC.

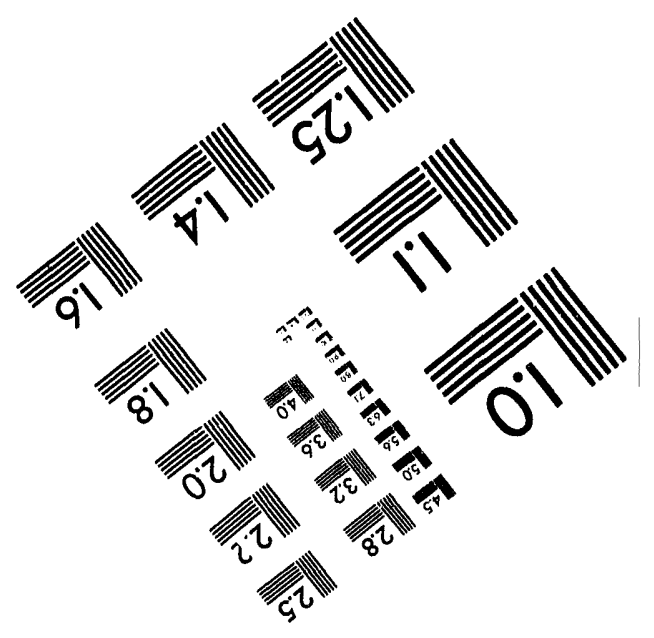



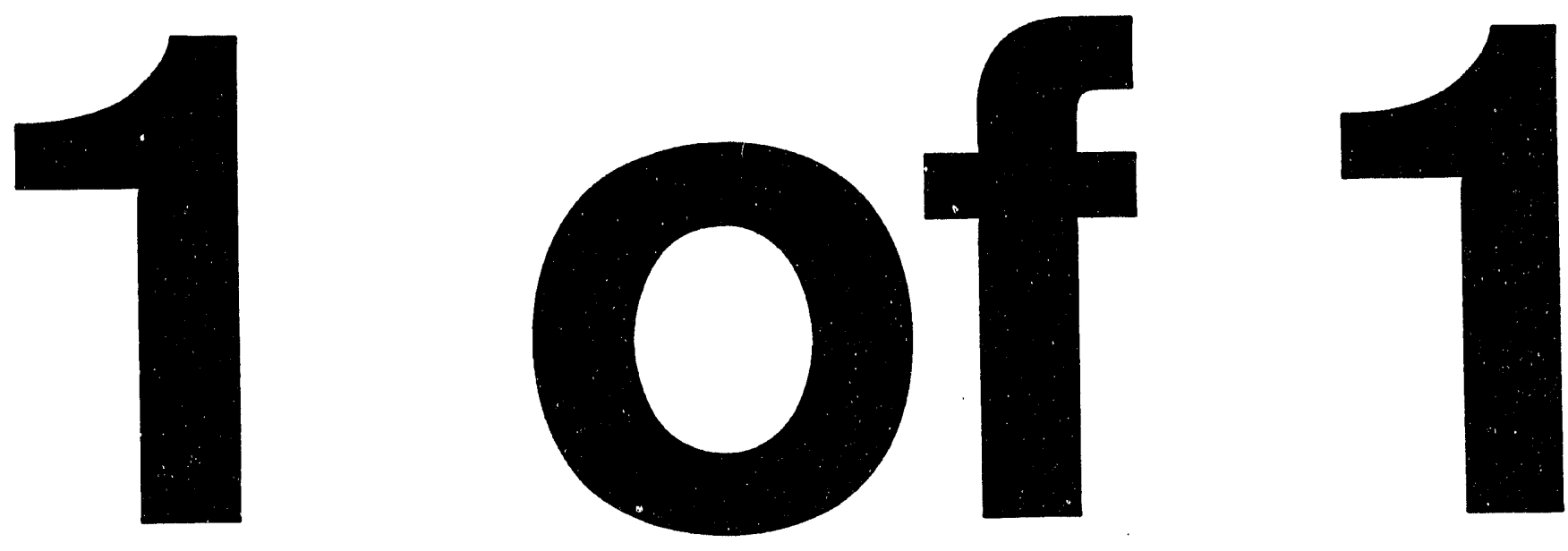


\section{TLSI W614 Laser Characterization}

Kansas City Division

M. N. Rector

KCP-613-5397

Published August 1994

Approved for public release; distribution is unlimited. 


\section{DISCLAIMER}

This report was prepared as an account of work sponsored by an agency of the United States Government. Neither the United States Government nor any agency thereof, nor any of their employees, makes any warranty, express or implied, or assumes any legal liability or responsibility for the accuracy, completeness, or Luefulness of any information, apparatus, product, or process disclosed, or represents that its use would not infringe privately owned rights. Reference herein to any specific commercial product, process, or service by trade name, trademark, manufacturer, or otherwise, does not necessarily constitute or imply its endorsement, recommendation, or favoring by the United States Government or any agency thereof. The views and opinions of authors expressed herein do not necessarily state or reflect those of the United States Government or any agency thereof.

Printed in the United States of America.

This report has been reproduced from the best available copy.

Available to DOE and DOE contractors from the Office of Scientific and Technical Information,

P. O. Box 62, Oak Ridge, Tennessee 37831; prices available from (615) 576-8401, FTS 626-8401.

Available to the public from the National Technical information Service, U. S. Department of Commerce, 5285 Port Royal Rd., Springfield, Virginia 22161.

A prime contractor with the United States Department of Energy under Contract Number DE-ACO4-76-DP00613.
AlliedSignal Inc. Kansas City Division P.O. Box 419159 Kansas City, Missourl 64141-6159 
KCP-613-5397

Distribution Category UC-706

Approved for public release; distribution is unlimited.

\section{TLSI W614 LASER CHARACTERIZF TION}

M. N. Rector

Published August 1994 


\section{Contents}

$\begin{array}{ll}\text { Section } & \text { Page }\end{array}$

Abstract $\ldots \ldots \ldots \ldots \ldots \ldots \ldots \ldots \ldots \ldots \ldots \ldots \ldots \ldots \ldots \ldots \ldots$

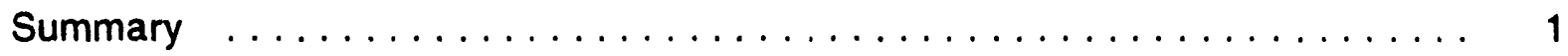

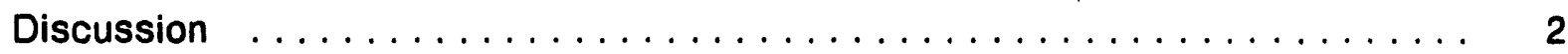

Scope and Purpose $\ldots \ldots \ldots \ldots \ldots \ldots \ldots \ldots \ldots \ldots \ldots \ldots \ldots \ldots \ldots \ldots$

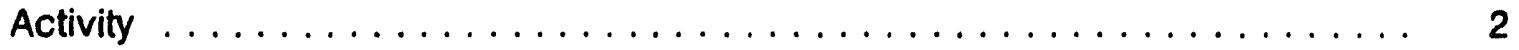

Trimming Discontinuities $\ldots \ldots \ldots \ldots \ldots \ldots \ldots \ldots \ldots \ldots$

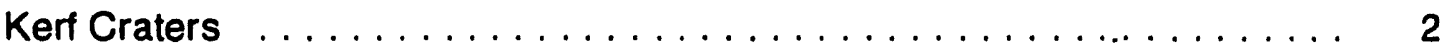

Optimization for Thick Film Resistors $\ldots \ldots \ldots \ldots \ldots \ldots$

Visual Criteria for Thick Film Resistors $\ldots \ldots \ldots \ldots \ldots$

Optimization for Thin Film Resistors $\ldots \ldots \ldots \ldots \ldots \ldots$

Vistial Criteria for Thin Film Resistors $\ldots \ldots \ldots \ldots \ldots \ldots$

W614 Laser Trim System Calibration $\ldots \ldots \ldots \ldots \ldots$

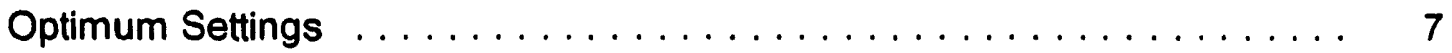

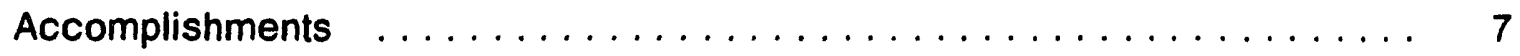

Appendices

A. Equipment Used $\ldots \ldots \ldots \ldots \ldots \ldots \ldots \ldots \ldots \ldots \ldots \ldots$

B. Resistor Test Patterns $\ldots \ldots \ldots \ldots \ldots \ldots \ldots$

C. Trim Increment Start Positioning $\ldots \ldots \ldots \ldots \ldots \ldots \ldots \ldots$

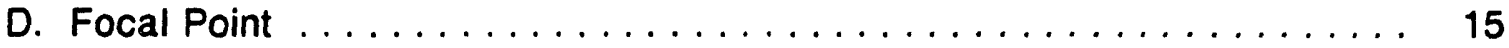

E. Thick Film Characterization Data $\ldots \ldots \ldots \ldots \ldots \ldots \ldots$

F. Thin Film Characterization Data $\ldots \ldots \ldots \ldots \ldots \ldots \ldots \ldots \ldots$ 


\section{Tables}

Number

Page

1 Laser Settings for Thick Film Characterization $\ldots \ldots \ldots \ldots \ldots \ldots \ldots$

2 Laser Settings for Thin Film Characterization $\ldots \ldots \ldots \ldots \ldots \ldots \ldots \ldots$

3 Optimum Laser Trim Settings $\ldots \ldots \ldots \ldots \ldots \ldots \ldots \ldots \ldots$ 


\section{Abstract}

The TLSI W014 Laser Trim System, which is a part of the TE5599 Tester, was characterized to find the optimum operating conditions for production trimming of thick and thin film resistors. Operating variables include average laser power, Q-rate, bite size, laser beam focal point, and trim increment start conditions. Operating conditions were found that support a life-stable resistance and a predictable trimming process.

\section{Summary}

In order to characterize the trimming process on the TLSI W614 Laser Trim System, thick and thin film resistors were trimmed while average laser power at the trim surface, Q-rate, and bite size were varied.

The resistance drift was monitored to find the conditions that resulted in the most stable trimmed resistors. The optimum operating conditions were found and recorded.
A larger sample was trimmed at the optimurn operating conditions to verify the results and study the effect of a varying focus. Resistors trimmed within the \pm 34 mil depth of focus did not exhibit significant variation in drift.

Trim increment start conditions were studied to find the best settings for a continuous, and thus predictable, trimming process as well as to reduce craters in the resistor trim kerfs. 


\section{Discussion}

\section{Scope and Purpose}

The results of this experiment are specific only to the TLSI W614 Laser Trim System and the resistance films used. The goals of this experiment were (1) to find the best trim increment start conditions; (2) to optimize laser power, bite size, and Q-rate by looking at relative resistance drift; and (3) to study the effect of a varying focus. Absolute resistance drifts are dependent on resistor geometry, trim geometry, and resistance film, which were held constant. An attempt was made to use worst case geometries.

\section{Activity}

The resistors trimmed for this characterization were on 3.75 in. $\times 4.5$ in. substrates in a repetitive test pattern (see Appendix $A$ for resistance films used and Appendix B for test patterns). Trim increment start positioning (see Appendix C) was studied first so that any changes made as a result of the findings could be completed before proceeding to the optimization study. The focal point and associated error (see Appendix D) were found last. However, this study shows that the focal error effects are insignificant.

\section{Trimming Discontinulties}

Discontinuities of the trimmed variable versus programmed trim increment length were found to correspond to discontinuities in the actual trim increment length. Whether or not these discontinuities occurred was dependent on the method of determining the start position of each trim increment. A calculated start position is simply the stop position of the previous increment specified by the software. An "as-read" start position is the stop position of the previous increment as read by the galvo sensors. The stop position is always calculated.

When calculated rather than as-read start positions were used, discontinuities were significantly reduced. The discontinuities of the as-read method are caused by a random over-travel of the galvos at the end of the previous increment. Using a calculated start and stop position reduces the discontinuities, but the as-read start position should be used after long increments ( $>150$ mils) because of the potential for physically disconnected increments ("gaps"). See Appendix C for a plot that compares the discontinuities of the two methods.

Notice that there are still discontinuities using the calculated start position. These only occur when switching to or from the smallest increment length (0.5 mils). During normal production trimming, the increment length only sequentially decreases, resulting in undershooting, which is recoverable. Overshooting is potentially unrecoverable but will not occur during normal trimming.

\section{Kerf Craters}

Craters in the trim kerfs were virtually eliminated by using a trim increment "start delay" of 2 ms. Start delays as long as $15 \mathrm{~ms}$ were used without a problem, but with significantly ionger start delays, there is a risk that increments will not be physically connected, thereby defeating the precaution of overlapping increments. Lag factor is defined in the W614 software as 
the "time period at the end of a laser trim during which the laser will continue to fire and the beam continue to move." Any lagfactor greater than zero caused increasingly large errors in increment length.

\section{Optimization for Thick Film Resistors}

All thick film resistors were two-square resistors, $100 \times 50$ mils, made from 10 , 100,1000 and $10,000 \Omega / \square$ films. L-cuts were made penetrating $66 \%$ of the 50 mil dimension. See Appendix B for drawings of resistor test patterns. The second leg of the L-cut was made as a series of increments of decreasing length. The increment length was decreased by $50 \%$ each time until it was 0.5 mils. The final increment length was repeated so that the last two increments were 0.5 mils.

Three resistors were trimmed at each recipe. Of the three resistors, each was on a different substrate and in a different geometric orientation on the substrate so these effects could be averaged out. Average laser power at the trim surface was varied from the level that barely cleaned out the kerf (found by isolation resistance) to the level that caused excessive heat-affected zone (defined as 2 mils each side of the kerf after swabbing with alcohol). This was done at various Q-rates and bite sizes. The conditions used are listed in Table 1.

Bite size can be translated to trim speed by the following formula.

$$
\text { Trim Speed }=\text { Bite Size } \cdot \text { Q-rate }
$$

Immediately after the trimming, the resistance values were measured and saved. Resistance was measured again 24 hours later and again after 10 temperature cycles from -40 to $+125^{\circ} \mathrm{C}$. Temperature cycling accelerates resistor drift caused by mechanical stress (temperature coefficient of expansion). Resistance drift, defined as change in percent of initial resistance, was plotted as averages of the three samples versus laser power and bite size at each Q-rate to find the window of minimum drift (maximum stability). The data in Appendix $E$ show that a Q-rate of $3 \mathrm{kHz}$, a bite size of 0.122 mils, and a laser power of 1.4 watts is well within the optimum operating window for all resistance films. The films used are named in Appendix $A$, along with other equipment used in the project.

The optimum operating window had no upper power limit, which provides additional confidence. The absolute drifts are dependent on resistor geometry, trim geometry, and resistance film, which were all held constant. The intent of this experiment was to look at only relative drift so that laser power, bite size, and Q-rate could be optimized.

Next, the optimum operating condition noted above was used to trim 24 resistors at nominal focus, -20 mils, and +20 mils. The plot of kerf width versus Z-height in Appendix $D$ shows that the nominal focus was off by -11.8 mils during this experiment (independent of the type of resistor). The data and histograms in Appendix $E$ show that resistor drift is not adversely affected $1<1 \%$ difference in drift) by a focal error of 31.8 mils below focus (20 mils below nominal is 31.8 mils below focus because of the $-11.8 \mathrm{mil}$ error). The trend suggests that within the \pm 34 mil depth of focus, variances would be insignificant. Depth of focus is defined as the two points where the spot size increases by $10 \%$ from the spot size at focus. The direction of focal error is irrelevant.

\section{Visual Criteria for Thick Film} Resistors

Visual criteria are evaluated by the correlation between discernible visual 
Table 1. Laser Settings for Thick Film Characterization

\begin{tabular}{||c|l|l||}
\hline Q-rate (kHz) & $\begin{array}{c}\text { Bite Size } \\
\text { (mils) }\end{array}$ & \multicolumn{1}{|c|}{ Laser Power Levels (watts) } \\
\hline 2 & 0.080 & $0.18,0.46,0.76,1.06$, and 1.36 \\
\hline 2 & 0.122 & $0.31,0.8,1.3,1.78$, and 2.27 \\
\hline 2 & 0.180 & $0.3,0.81,1.36,1.9$, and 2.44 \\
\hline 2 & 0.250 & $0.37,1.03,1.72,2.41$, and 3.1 \\
\hline 3 & 0.080 & $0.22,0.6,0.97,1.35$, and 1.72 \\
\hline 3 & 0.122 & $0.31,0.70,1.10,1.49$ and 1.88 \\
\hline 3 & 0.180 & $0.42,0.96,1.5,2.05$, and 2.6 \\
\hline 3 & 0.250 & $0.56,1.16,1.76,2.36$, and 2.96 \\
\hline 4 & 0.080 & $0.21,0.57,0.94,1.3$, and 1.66 \\
\hline 4 & 0.122 & $0.31,0.81,1.3,1.83$, and 2.36 \\
\hline 4 & 0.180 & $0.43,1.07,1.69,2.33$, and 2.97 \\
\hline 4 & 0.250 & $0.57,1.36,2.16,2.96$, and 3.76 \\
\hline
\end{tabular}

characteristics of a trimmed resistor and the quality of the trim. Resistance film in the kerf (dirty kerfs) and excessive heataffected zone as visual criteria are associated with insufficient and ex:essive levels of energy per unit length of trim, respectively. A clean kerf is defined here as being greater than 1.3 mils wide at the bottom of the resistance film. If the kerf was clean, the resistor was within the window of acceptable stability. Dirty kerfs were also accompanied by a lack of isolation resistance, that is, a resistance less than $32 \mathrm{M} \Omega$ when trimmed completely in half.

If the heat-affected zone adjacent to the kerf was greater than 2 mils on each side of the kerf, it was considered excessive. To make a more objective determination of the heat-affected zone, it was measured after the resistor was swabbed with alcohol to remove any potentially deceptive blow off. Resistors that were trimmed with excessive heat-affected zone did not exhibit significant instability, but the visual criterion is given here so that an upper limit on energy can be established.

More simply stated:

1. If the kerf is dirty, the energy level was insufficient and the resistor may be unstable.

2. If the heat-affected zone is excessive, the energy level was excessive and the resistor may be unstable.

3. If neither of the above conditions is present, the resistor will be stable within the window of acceptable stability. 


\section{Optimization for Thin Film Resistors}

All thin film resistors were top hat resistors, $200 \times 20$ mils, made from $100 \Omega / \square$ film. Plunge cuts were made along the $200 \mathrm{mil}$ dimension to 10 mils from the end. See Appendix $B$ for drawings of resistor test patterns. Each cut was made as a series of increments of decreasing length. The increment length was decreased by $50 \%$ each time until it was 0.5 mils. The final increment length was repeated so that the last two increments were 0.5 mils.

Three resistors were trimmed at each recipe. Of the three resistors, each was in a different geometric orientation on the substrate to average out the effect. Average laser power at the trim surface was to be varied from the level that barely cleaned out the kerf (found by isolation resistance) to the level that caused excessive heat-affected zone. However, isolation resistance occurred at very low power levels and an excessive heataffected zone could not be easily discerned, so a power level range was selected to be at least a factor of three abovs and below the current level. The power levels used are listed in Table 2 below.

Immediately after trimming, resistance values were measured and saved. Resistance was measured again 24 hours later and again after 200 hours of life testing at $+150^{\circ} \mathrm{C}$. Theoretically, life testing accelerates resistor drift caused by chemical processes (mostly oxidation of tantalum). Resistance drift, defined as drift percentage from initial resistance, was plotted as averages of the three samples at each Q-rate to find the window of minimum drift (maximum stability). The data in Appendix $F$ show that a Q-rate of $3 \mathrm{kHz}$, a bite size of 0.122 mils, and a laser power of 0.4 watts is well within the optimum operating window.

Next, the optimum operating condition above was used to trim 48 resistors at nominal focus, -20 mils, +20 mils, and $+\mathbf{4 0}$ mils. The data and histograms in Appendix $F$ show that resistor stability is not adversely affected $(<1 \%$ difference in drift) by a focal error of 51.8 mils below focus ( 40 mils below nominal is 51.8 mils below focus because of the -11.8 mil error mentioned above). The first histograms are bimodal. This is because of the dependence on trim geometry. Resistors that were trimmed vertically were trimmed differently enough from those that were trimmed horizontally to see a $0.5 \%$ difference in drift.

The histograms of resistors trimmed with common geometric orientation (vertical) are not bimodal. In contrast to thick film, the mean improves as the focal error moves to 51.8 mils below the focal point. This is probably caused by a decreased energy per unit length of trim associated with a larger spot size. Less energy would theoretically reduce the drift as illustrated in the three-dimensional plot of drift at $3 \mathrm{kHz}$. Thick film 3-D plots are flatter and therefore would not suggest the same dependence on spot size. Depth of focus

Table 2. Laser Settings for Thin Film Characterization

\begin{tabular}{||c|c|c||}
\hline \hline Q-rate (kHz) & Bite Size (mils) & Laser Power Levels (watts) \\
\hline 2,3 , and 4 & $0.08,0.122,0.18$, and 0.25 & $0.10,0.24,0.366,0.90$, and 1.5 \\
\hline
\end{tabular}


is defined as the two points where spot size increases by $10 \%$ from the spot size at focus. The direction of focal error is ir:elevant.

The difference between the average drift of untrimmed resistors and vertically trimmed resistors with these parameters is $0.5 \%$. This does not satisfy the $0.1 \%$ absolute drift requirement because worst case geometries were used and the intent of this experiment was only to optimize laser power, bite size, and Q-rate. Only relative drifts need to bo considered to find the optimum settings.

\section{Visual Criteria for Thin Film Resistors}

Visual criteria are evaluated by the correlation between discernible visual characteristics of a trimmed resistor and the quality of the trim. Resistance film in the kerf (dirty kerts) and excessive heataffected zone as visual criteria are associated with insufficient and excessive levels of energy per unit length of trim, respectively. If the kerf appeared dirty (resistance film not completely removed) the resistor was outside of the window of acceptable stability. Dirty kerts were also accompanied by a lack of isolation resistance, that is, a resistance less than $32 M \Omega$ when trimmed completely in half.

A heat-affected zone could not be easily discerned for thin film resistors without special efforts. For this reason, the depth of the kert in thin film resistors can be used as a visual criterion for excessive level of energy per unit length of trim.

It was found that the drifts of thin film resistors with kert depths of 0.2 mils were only $0.3 \%$ larger than optimum. Note that 0.2 mils is two orders of magnitude thicker than the thin film resistors which are roughly $500 \AA$. This depth corresponds to a Q-rate of $3 \mathrm{kHz}$, a bite size of 0.122 mils, and a laser power of 1 watt. From this we can safely define 0.2 mils kerf depth as a visual criterion for excessive energy. More conservative visual criteria than those described above are a "slight flowing of the substrate material" and no groove in the substrate at all.

More simply stated:

1. If the kerf is dirty, the energy level was insufficient and the resistor may be unstable.

2. If the kerf depth is greater than 0.2 mils, the energy level was excessive and the resistor may be unstable.

3. If neither of the above conditions is present, the resistor will be stable within the window of acceptable stability.

\section{W614 Laser Trim System Calibration}

This characterization was done after successful calibration in accordance with two procedures and during one calibration interval for each. One procedure calibrates power, Q-rate, and bite size. Absolute accuracies for these parameters are pertinent. The other procedure calibrates the HP3.458A for measuring resistance.

Resistance drift measurements are more accurate than reported in the tester CP because only repeatability (not accuracy) is relevant and because this entire characterization was done during one calibration interval. The inaccuracies of the laser step attenuators $( \pm 10 \%$ worst case) need to be considered when looking at laser power levels specified in the plots of resistor drift versus power level and bite size. The power levels that were used to trim large samples at the optimum settings were not affected by the inaccuracies of the laser step attenuators. 


\section{Optimum Settings}

The optimum settings are listed in Table 3 below. Optimum settings for laser power, Q-rate, and bite size have not changed from those that have been in use on the TE5599. However, prior to this study, the TE5599 had been using an "as-read" start position and a start delay of $0 \mathrm{~ms}$. The optimum settings for these variables have changed to those in Table 3.

\section{Accomplishments}

The optimal operating parameters for the TLSI W614 Laser Trim System were found for production trimming of thick film and thin film resistors. The effects of focus were verified to be insignificant within the \pm 34 mil depth of focus. Increment start parameters were established for a predictable trimming process and reduced cratering.

Table 3. Optimum Laser Trim Settings

\begin{tabular}{||l|l|}
\hline System Variables & Optimum Settings \\
\hline $\begin{array}{l}\text { Average Laser Power } \\
\text { at the Trim Surface }\end{array}$ & $\begin{array}{l}1.4 \text { Watts for Thick Film; } \\
0.4 \text { Watts for Thin Film }\end{array}$ \\
\hline Q-rate & $3 \mathrm{kHz}$ \\
\hline Bite Size & 0.122 mils \\
\hline Start Delay & $2 \mathrm{~ms}$ \\
\hline Lag Factor & $0 \mathrm{~ms}$ \\
\hline Start Position & Calculated (as-read after $>150$ mil trims) \\
\hline
\end{tabular}




\section{Appendix A}

\section{Equipment Used}




\section{EQUIPMENT USED}

TLSI W614 Laser Trim System

1. Hitachi monitor

2. Laser power supply

3. Laser trimmer (see TE5599 top drawing for details)

4. Computer
a. PC386 compatible (WYSE-386, P/N 3016SX-01)
b. Coprocessor
C. Dual 90 MB Bernoulli removable disk drive (lomega S0700425)
d. 5.25 in. floppy drive
e. DOS 5.0
f. Microsoft $\mathrm{C}$, programming language

TE5599 Tester

1. HP3458A Multimeter with 4-wire ohms capability

2. HP3488 Scanner

3. TE5599-W98 Cable

4. TE5599-W99 Cable

Probe Cards

1. Thick film: MRSI, 103720 Rev. 2 with Kelvin probes

2. Thin film: Teledyne TAC, C103720 Rev. 2 with Kelvin probes

Thick Film Resistors

1. Two-square

2. $50 \times 100$ mils

3. $10 \Omega / \square$; Du Pont Number 1610

4. $100 \Omega / \square$; Du Pont Number 1421

5. $1000 \Omega / \square$; Du Pont Number 1431

6. 10,000 $\Omega / \square$; Du Pont Number 1441

7. Screen printed on $\mathbf{2 5}$ mil alumina substrate Thin Film Resistors

1. Top hat

2. $20 \times 200$ mils

3. $100 \Omega / \square$; tantalum nitride

4. Deposited on 25 mil alumina substrate 


\section{Appendix B}

\section{Resistor Test Patterns}




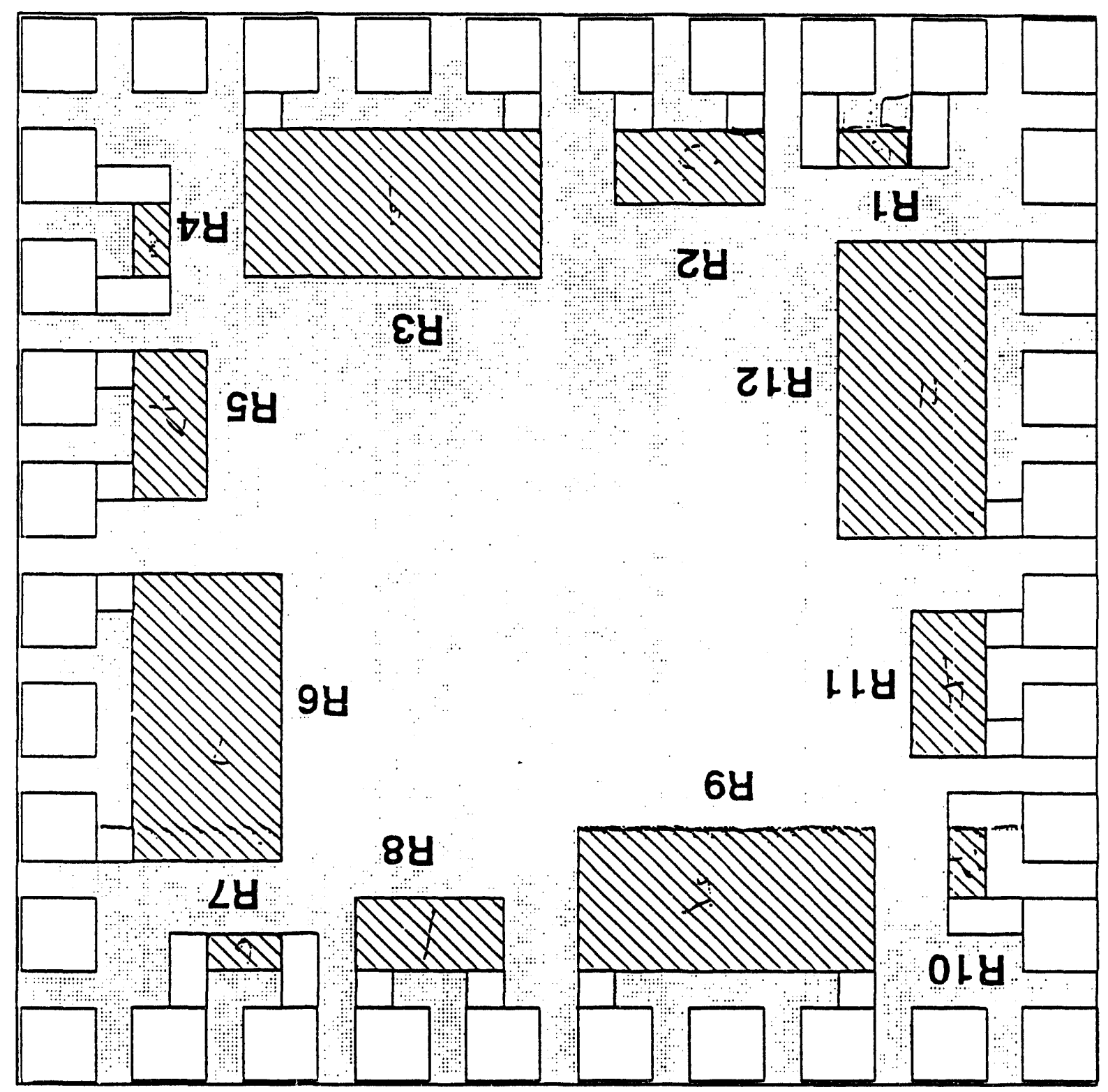

UO!pezü

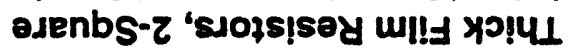


Thin Film Resistors, Top Hat
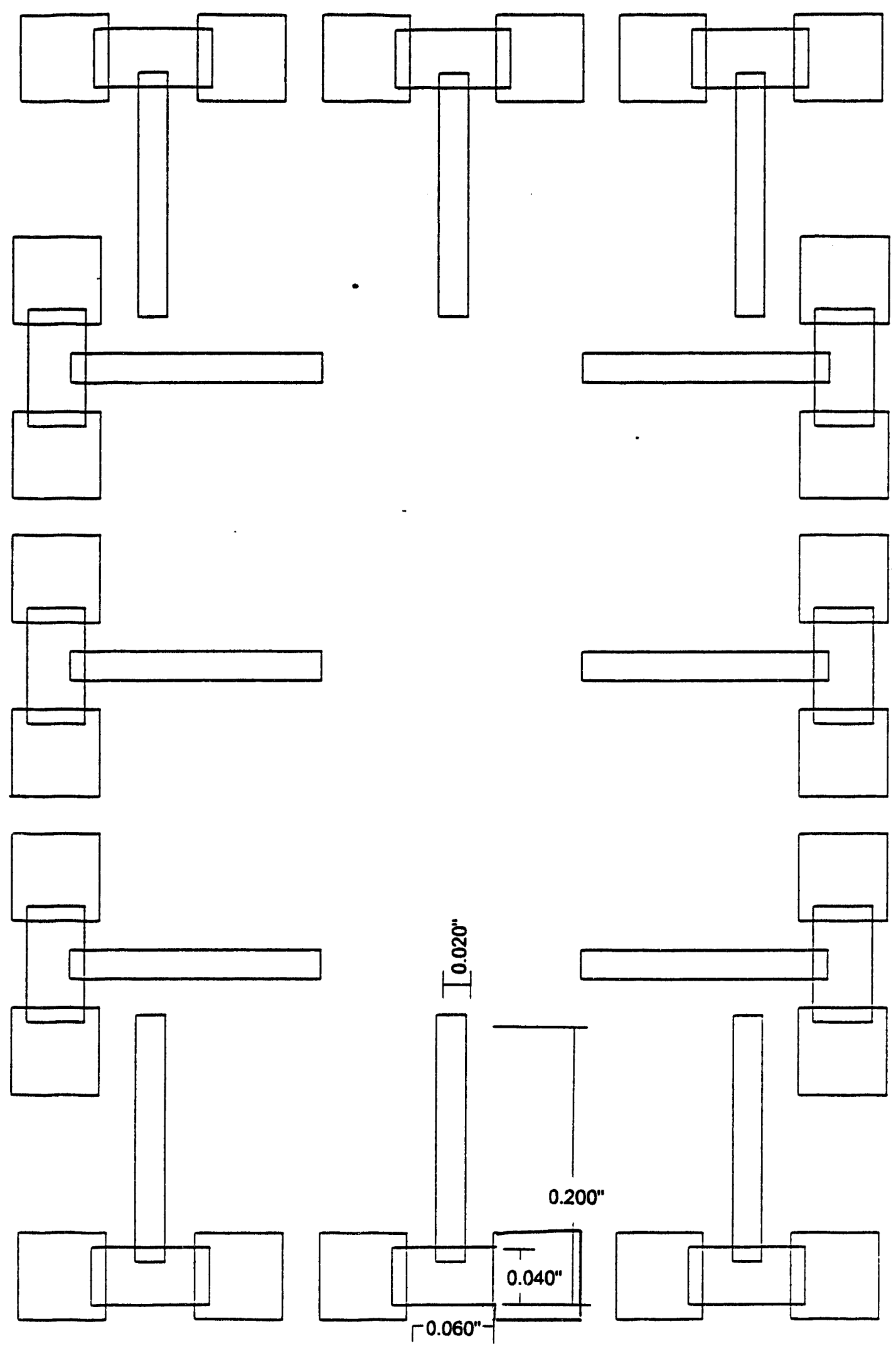


\section{Appendix C}

\section{Trim Increment Start Positioning}




\section{Effect of Start Position on Resistance vs. Programmed Trim Increments}

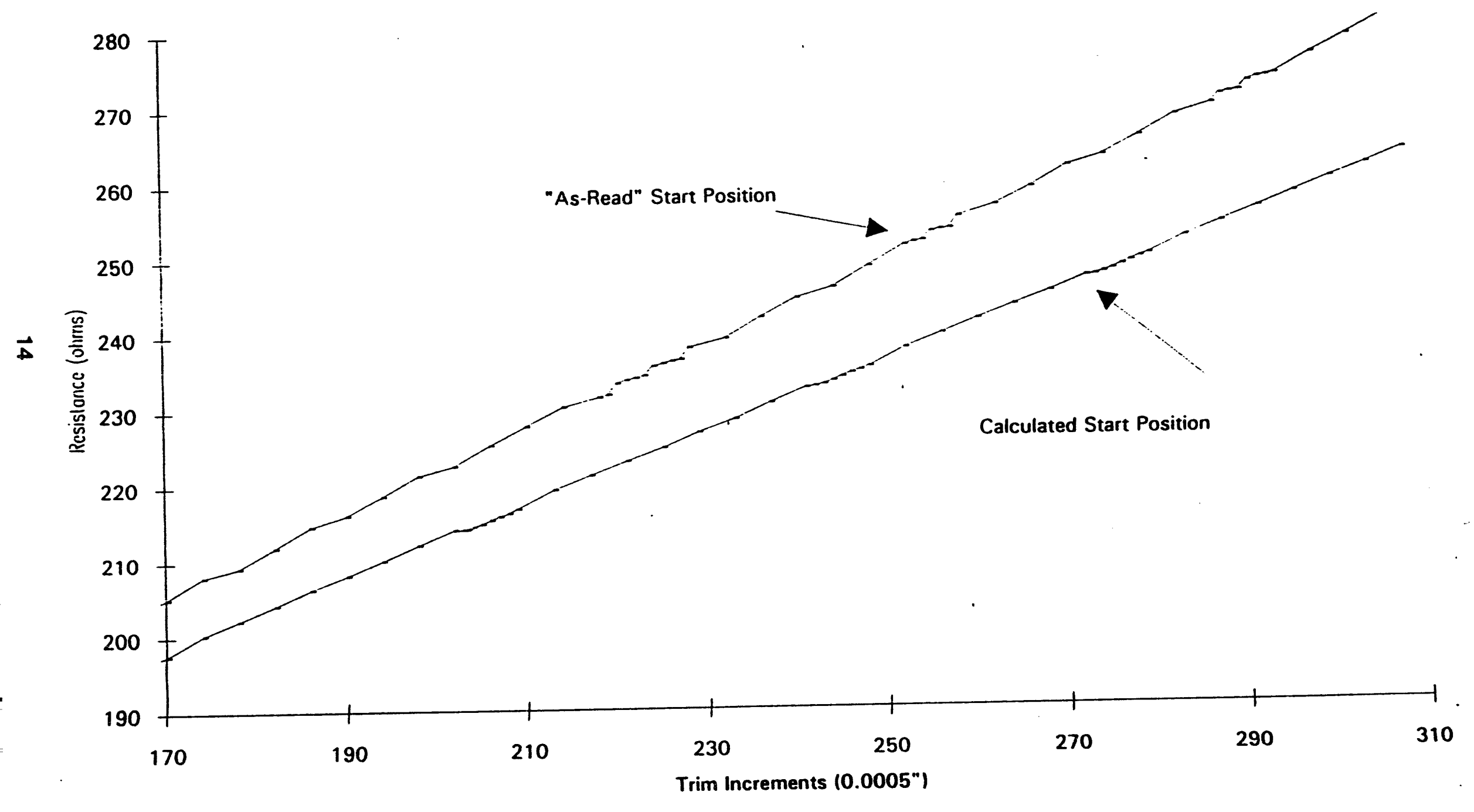


Appendix D

Focal Point 
Trim Width vs. $z$-height

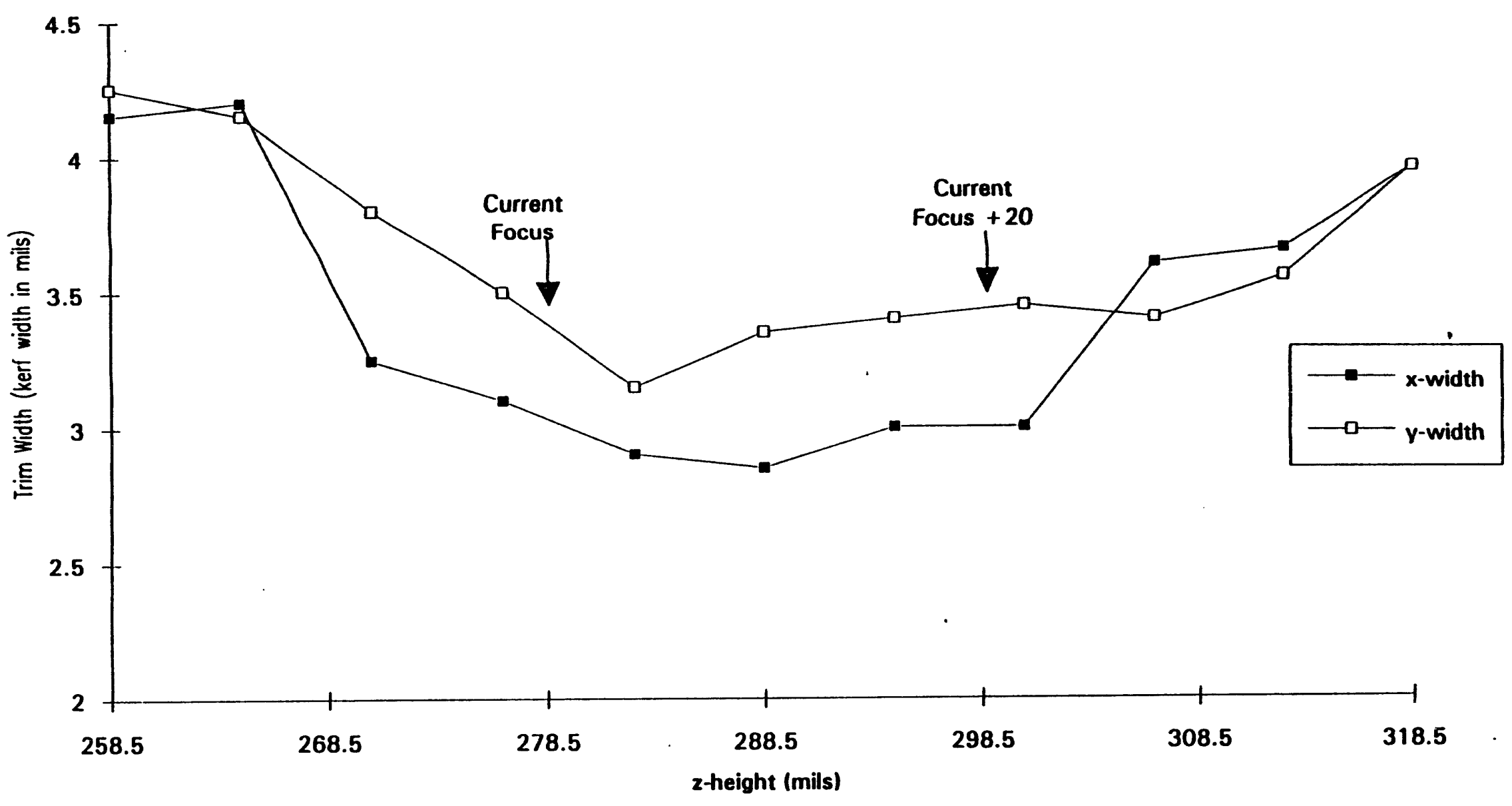




\section{Curve Fit to Average of Kerf Width vs.Table Height}

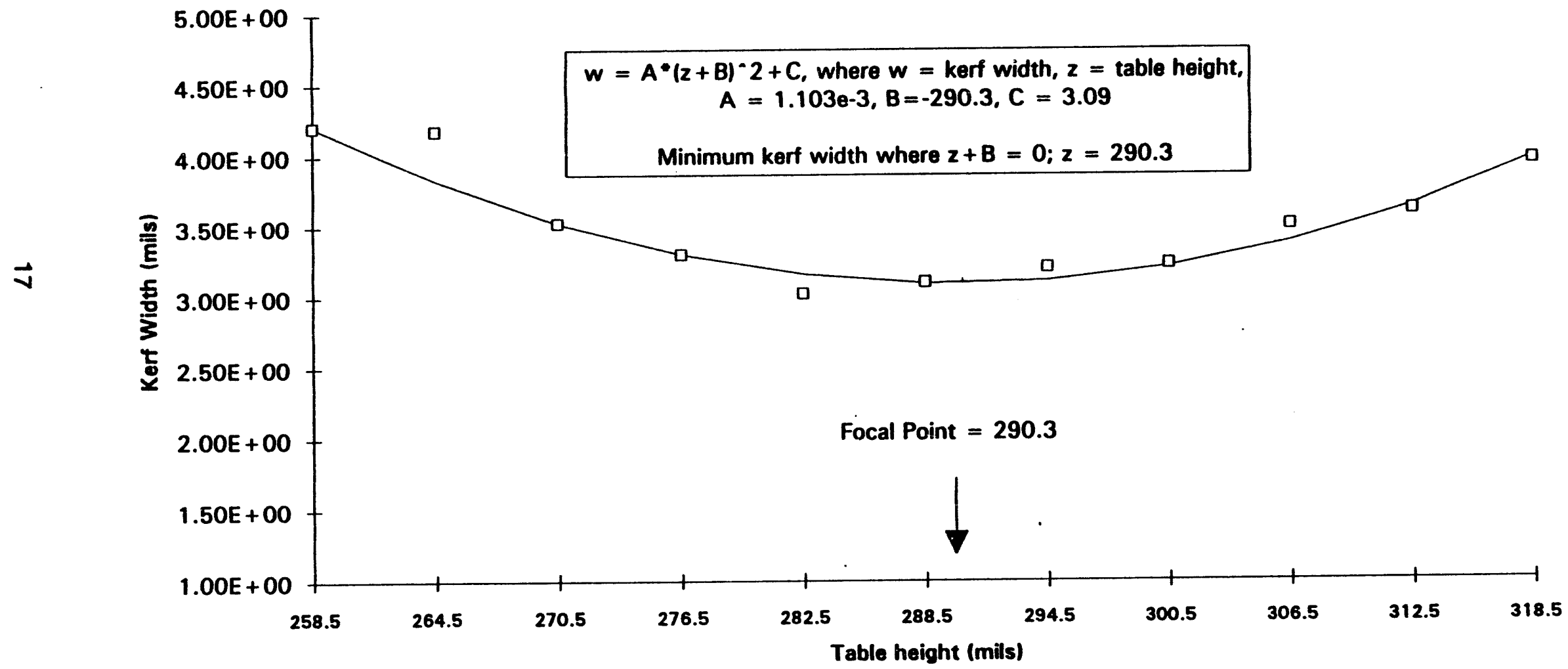




\section{Appendix E}

Thick Film Characterization Data 
24 Hour Resistance Drift (Q-rate $=2 \mathrm{kHz}, 10,000 \mathrm{ohms} / \mathrm{sq}$ )

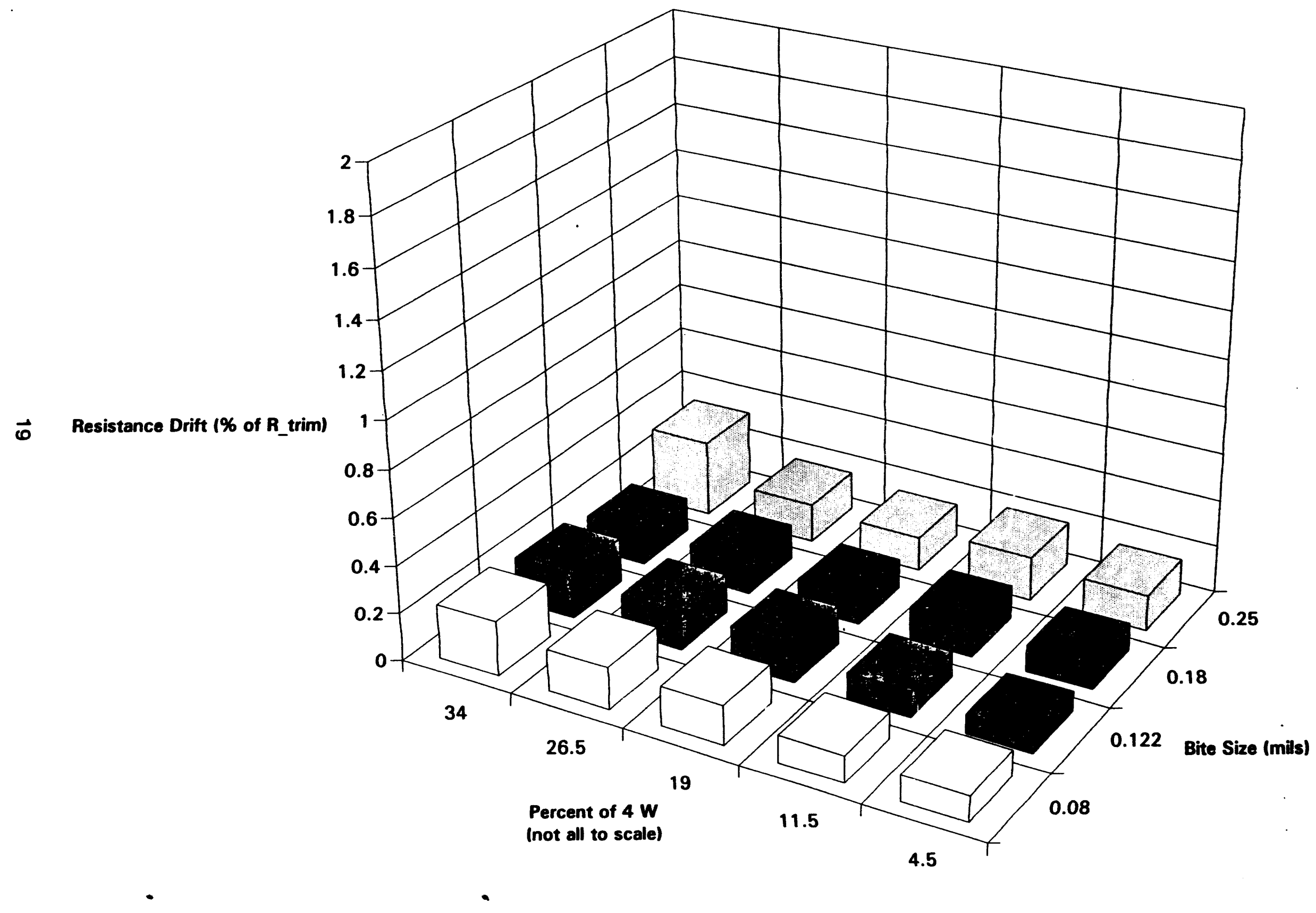




\section{Post Cycle Resistance Drift (Q-rate $=2$ kHz, 10,000 ohms/sq)}

Resistance Drift (\% of R_trim)

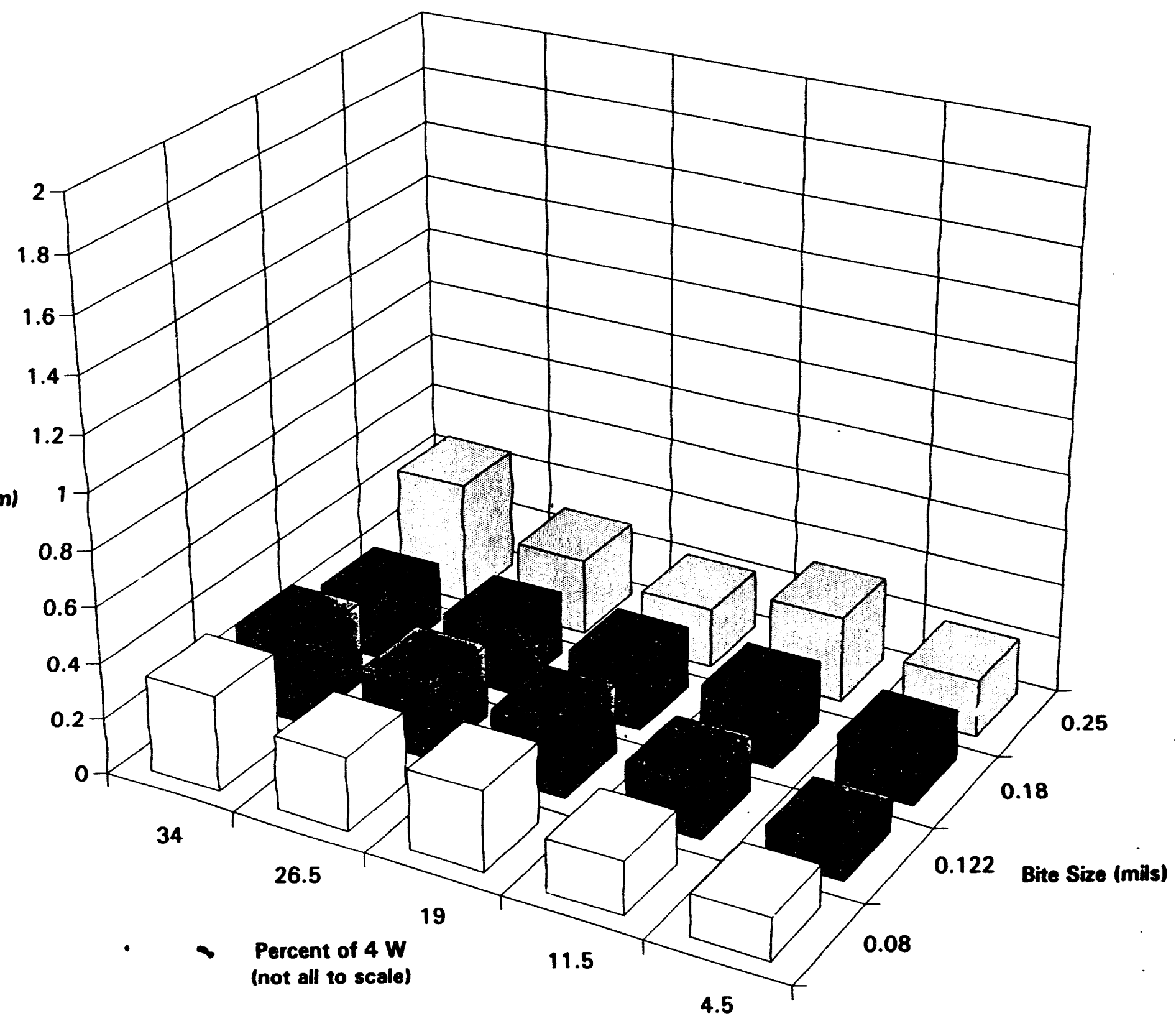


24 Hour Resistance Drift (Q-rate $=3$ kHz, 10,000 ohms/sq)

Resistance Drift (\% of R_trim)

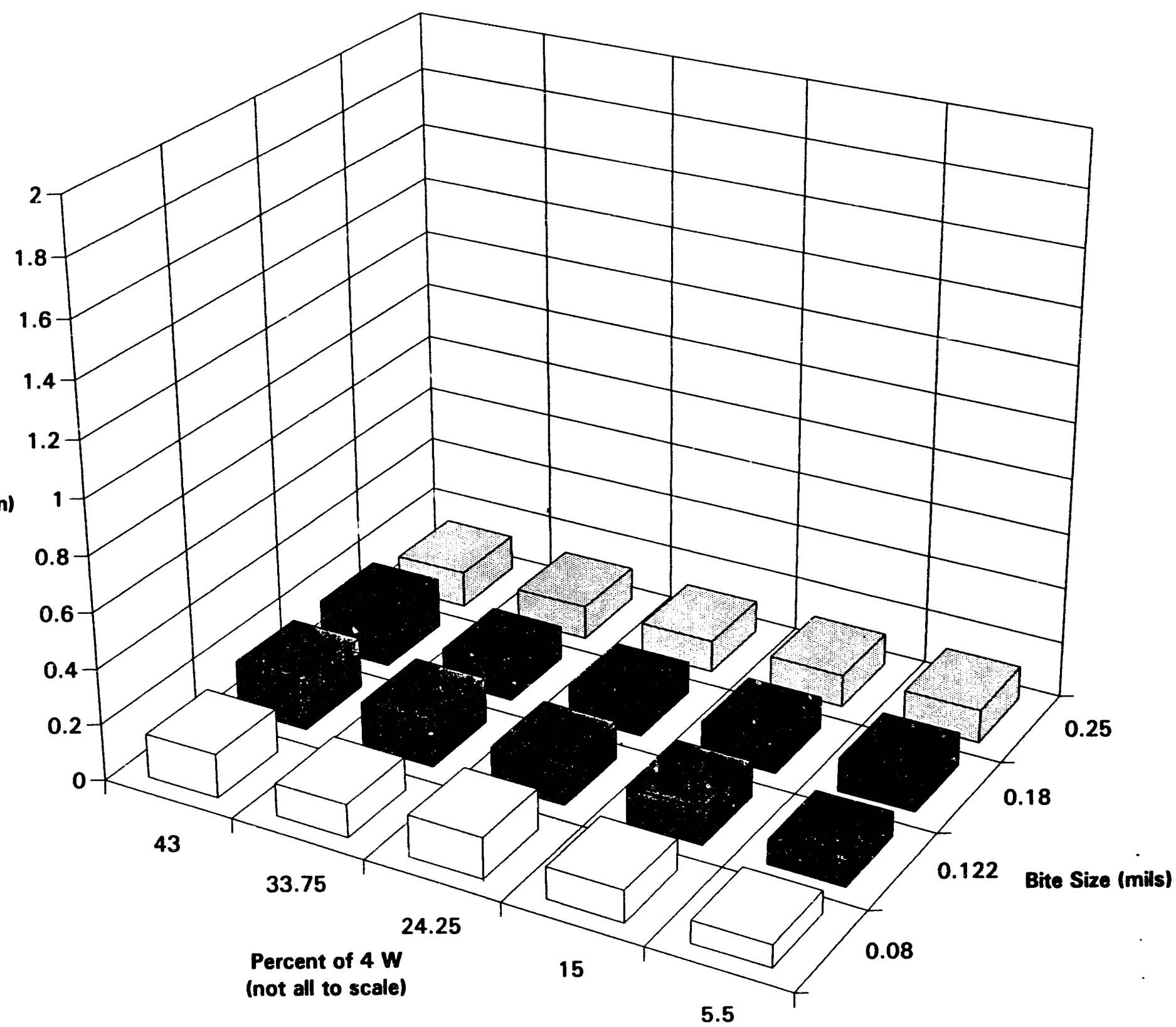


Post Cycle Resistance Drift (Q-rate $=3$ kHz, 10,000 ohms/sq)

N Resistance Drift (\% of R_trim)

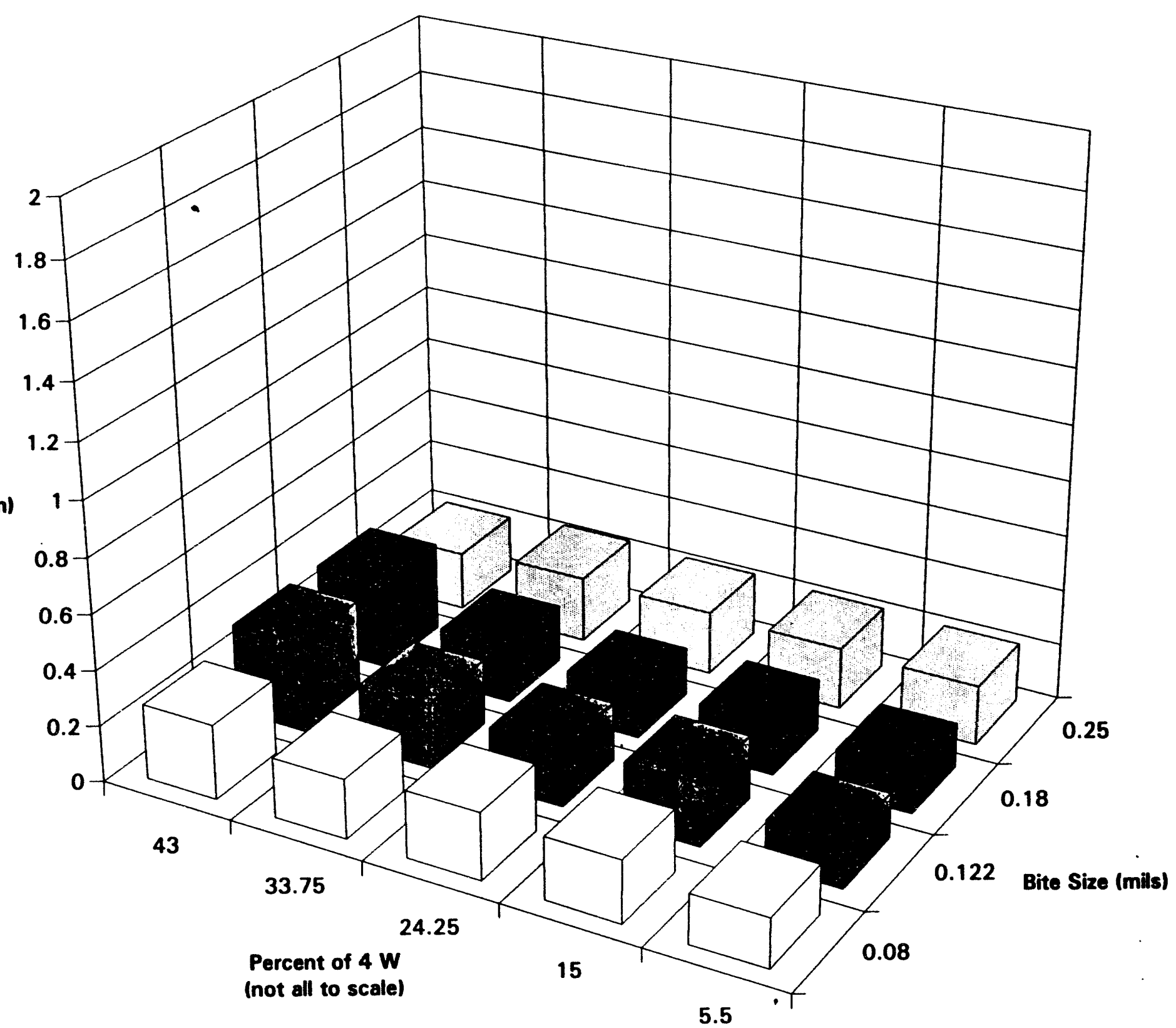


24 Hour Resistance Drift (Q-rate $=4$ kHz, 10,000 ohms/sq)

Resistance Drift (\% of R_trim)

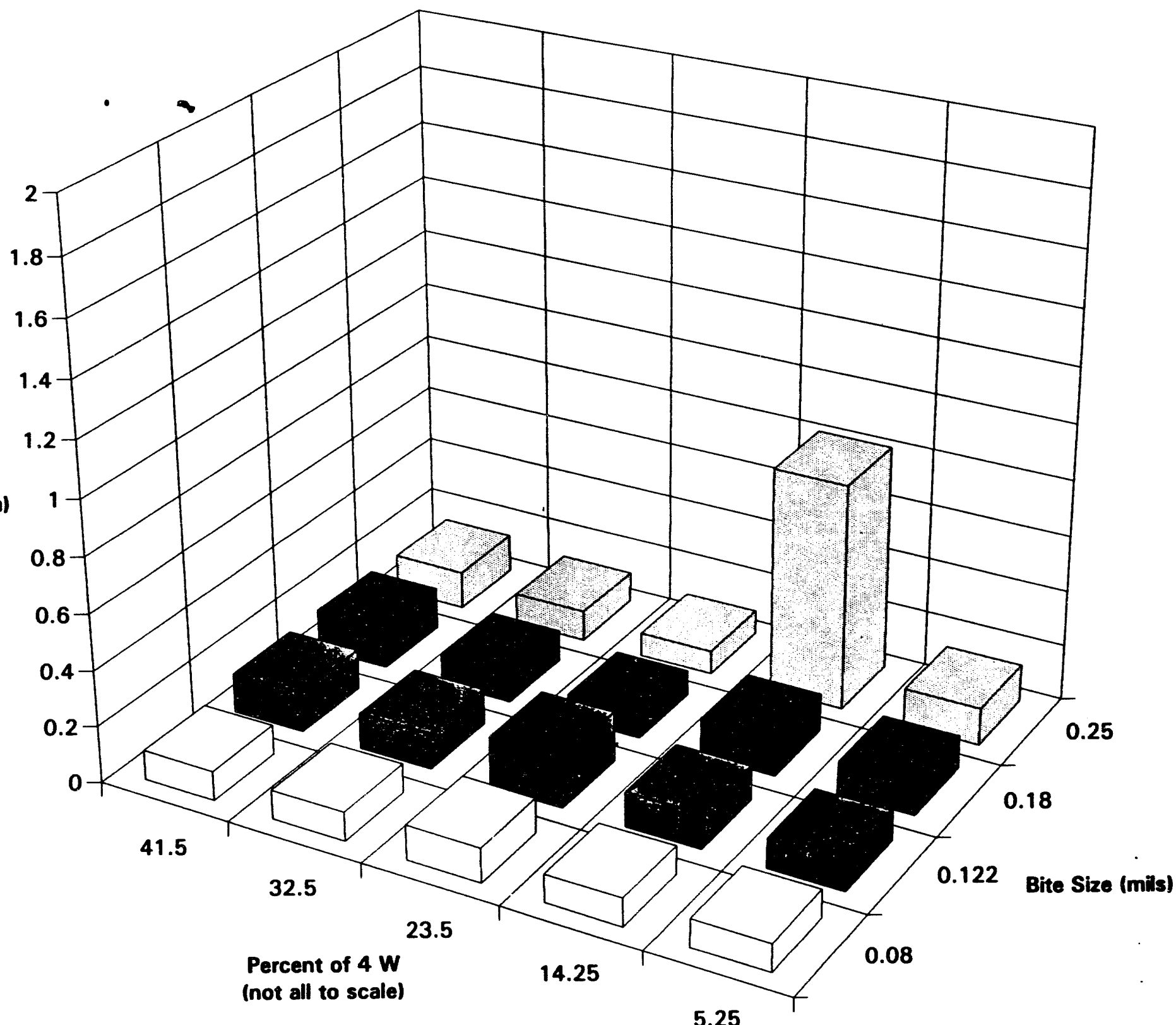


Post Cycle Resistance Drift (Q-rate $=4$ kHz, 10,000 ohms/sq)

N Resistance Drift (\% o R_trim)

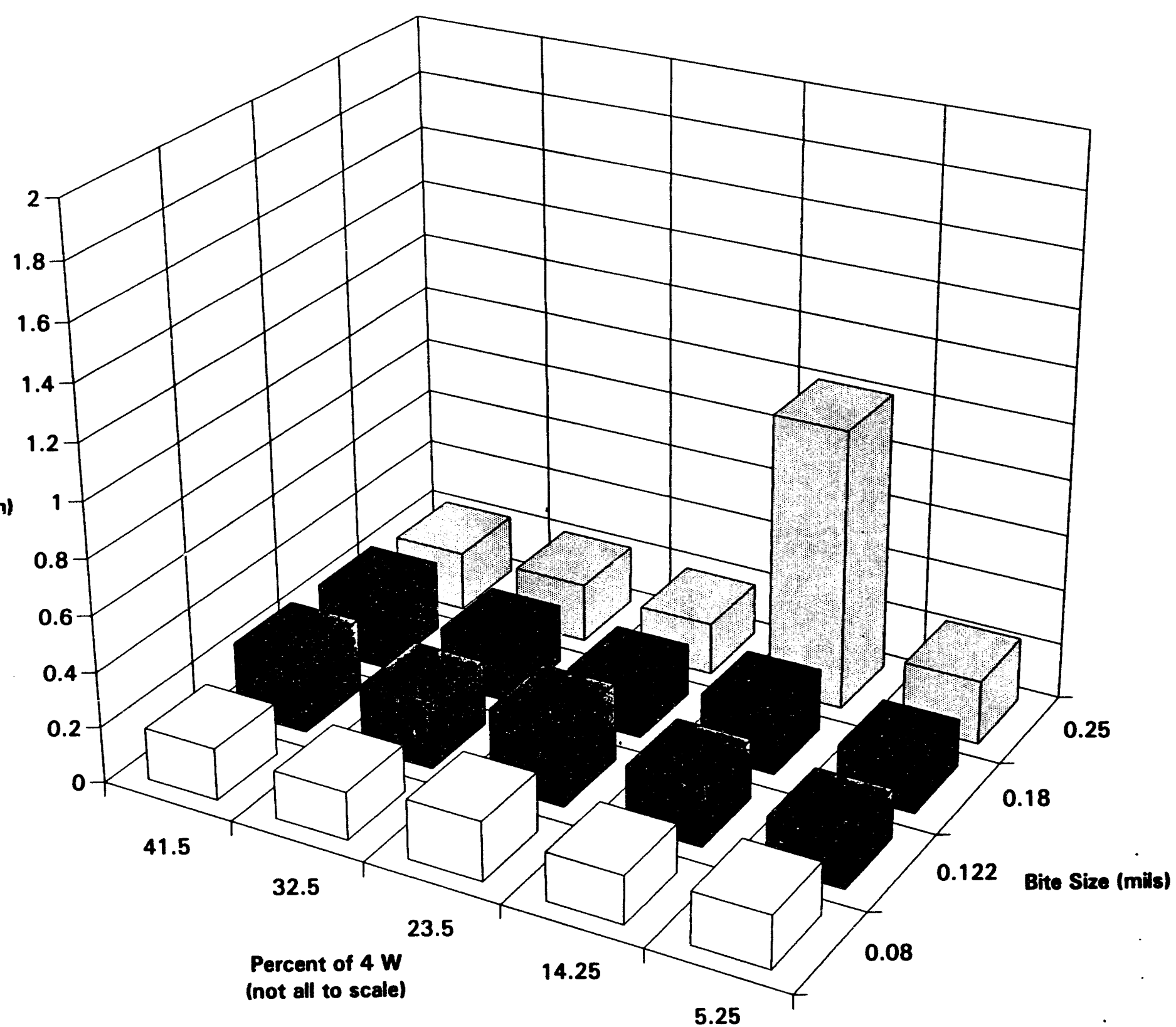


24 Hour Resistance Drift (Q-rate $=4$ kHz, 10,000 ohms/sq. 1 Sport Removed)

Resistance Drift (\% of R_trim)

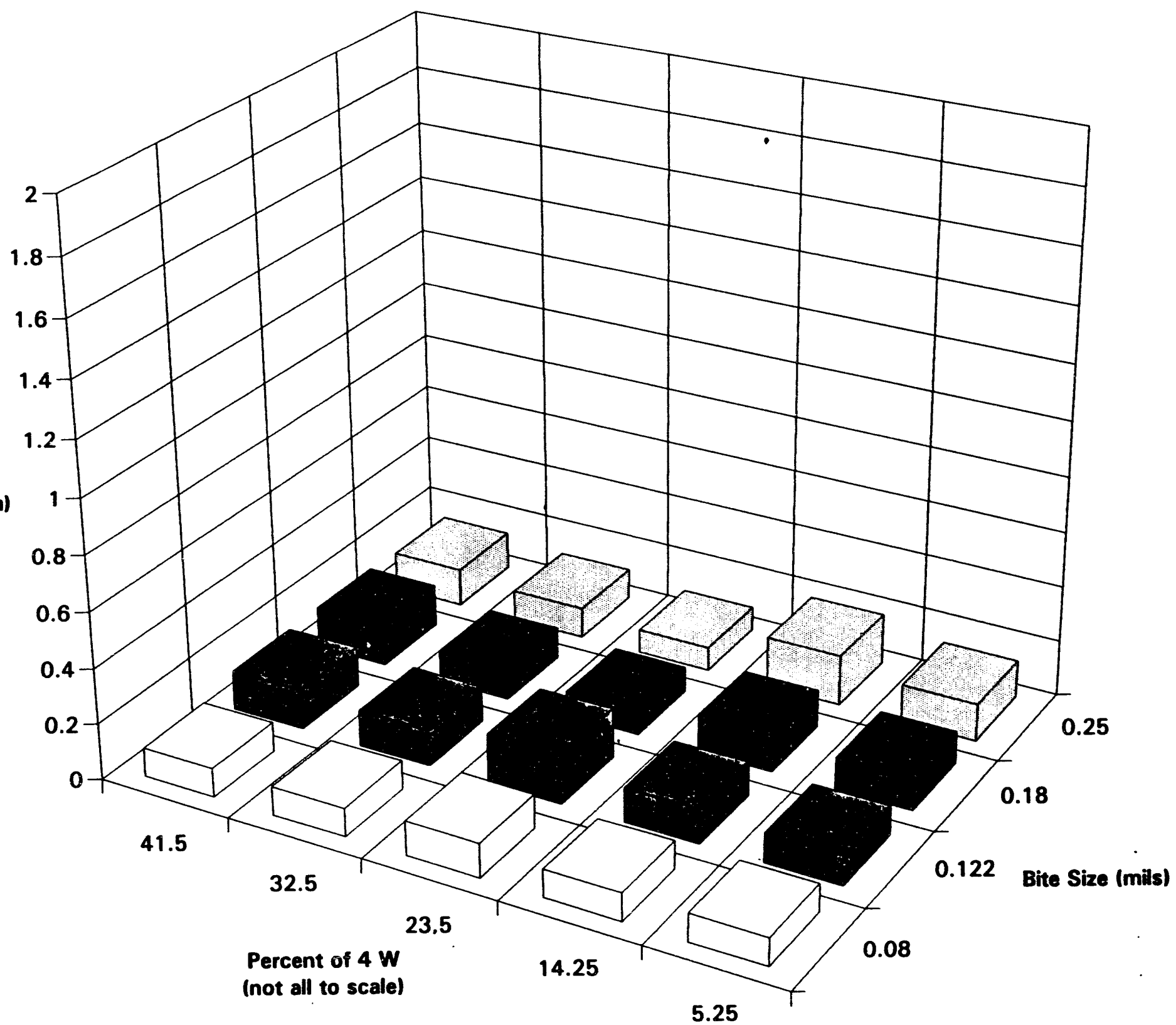


Post Cycle Resistance Drift (Q-rate $=4 \mathrm{kHz}, 10,000 \mathrm{ohms} / \mathrm{sq}, 1$ Sport Removed)

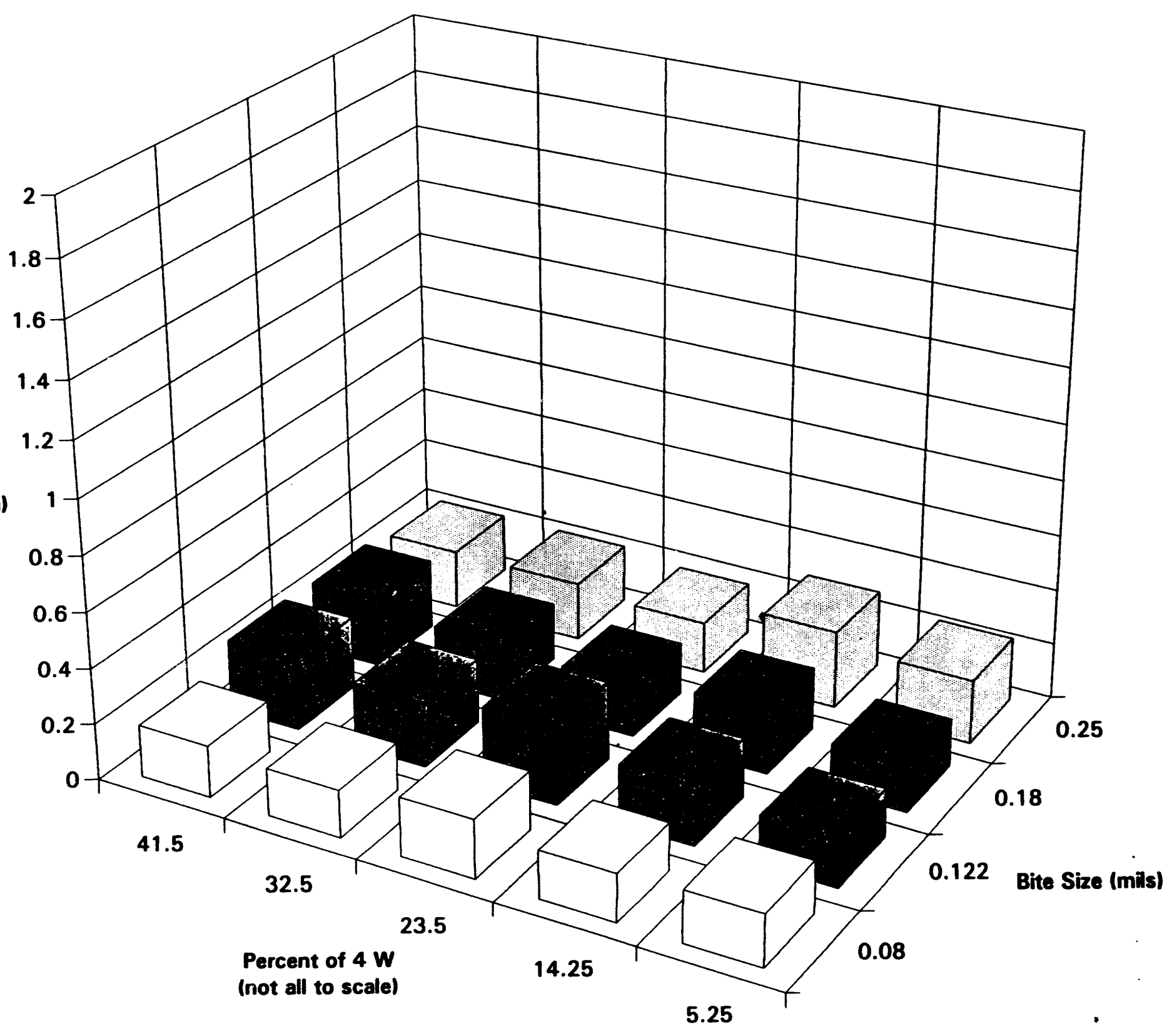


24 Hour Resistance Drift (Q-rate $=2$ kHz, 1000 ohms/sq, 11/18/93)

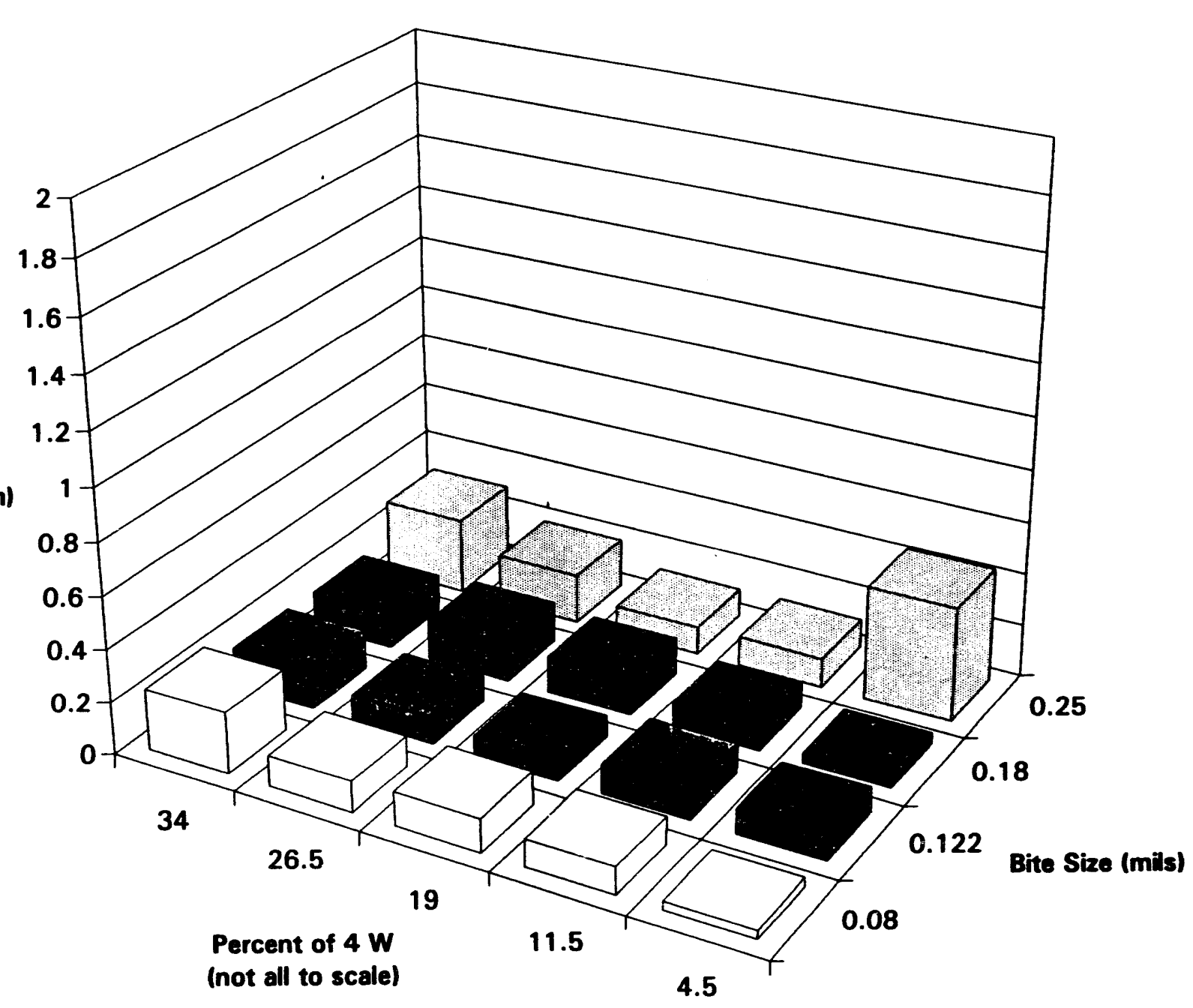


24 Hour Resistance Drift (Q-rate $=3$ kHz, 1000 ohms/sq, 11/18/93)

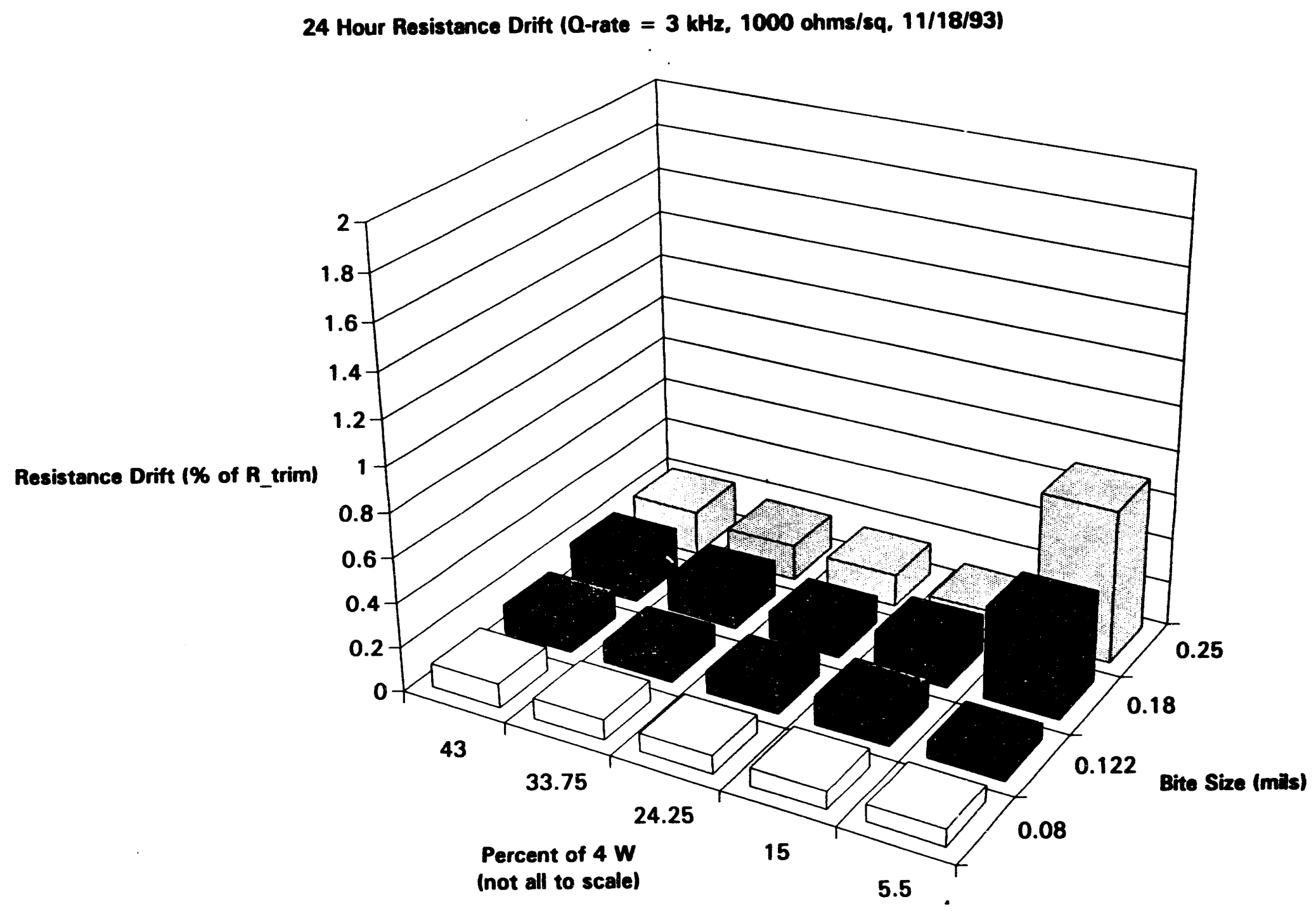


24 Hour Resistance Drift (Q-rate $=4$ kHz, 1000 ohms/sq, 11/18/93)

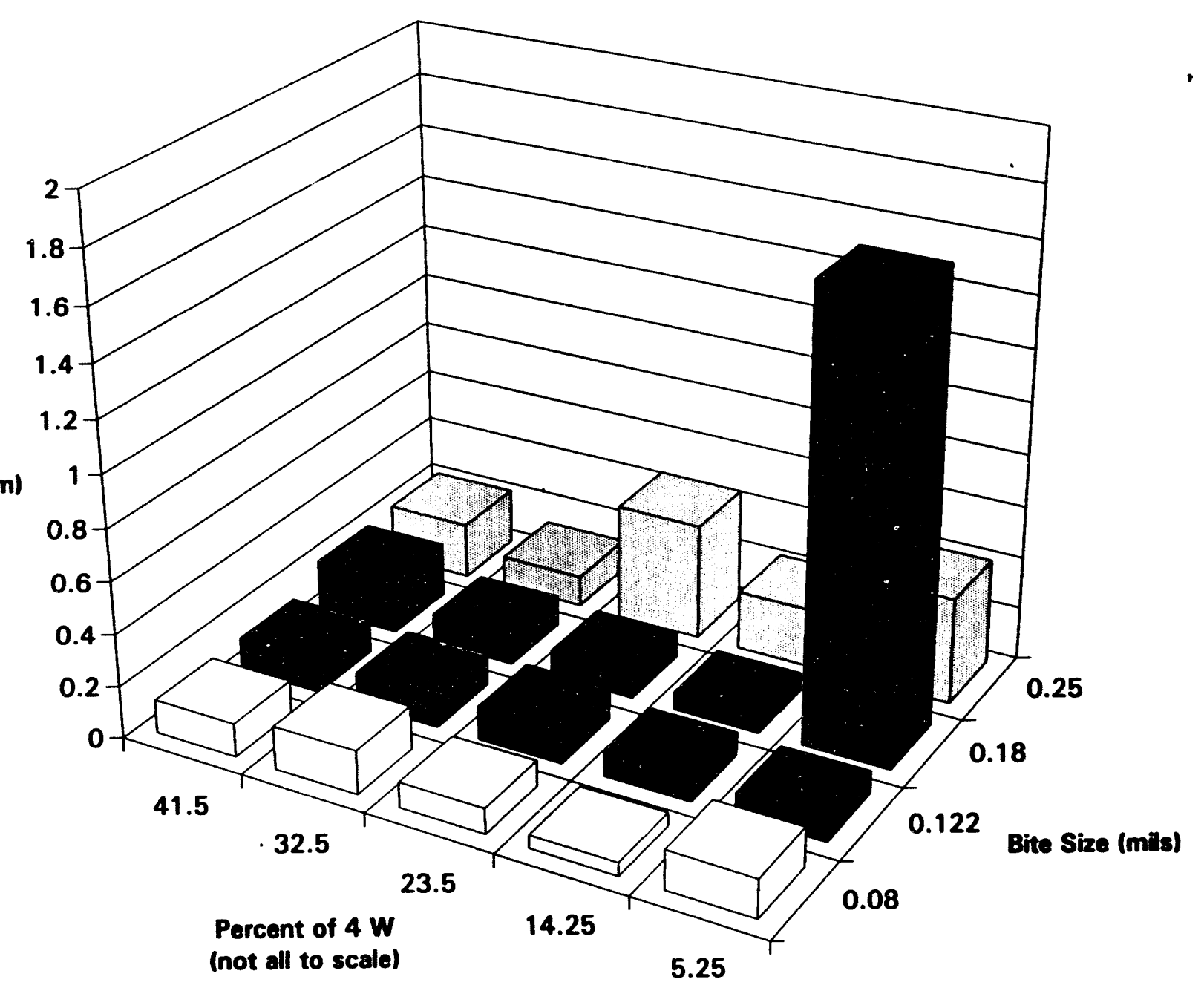


Post Cycle Resistance Drift (Q-rate $=2$ kHz, $1000 \mathrm{chms} / \mathrm{sq}, 11 / 18 / 93$ )

Resistance Drift (\% of R_trim)

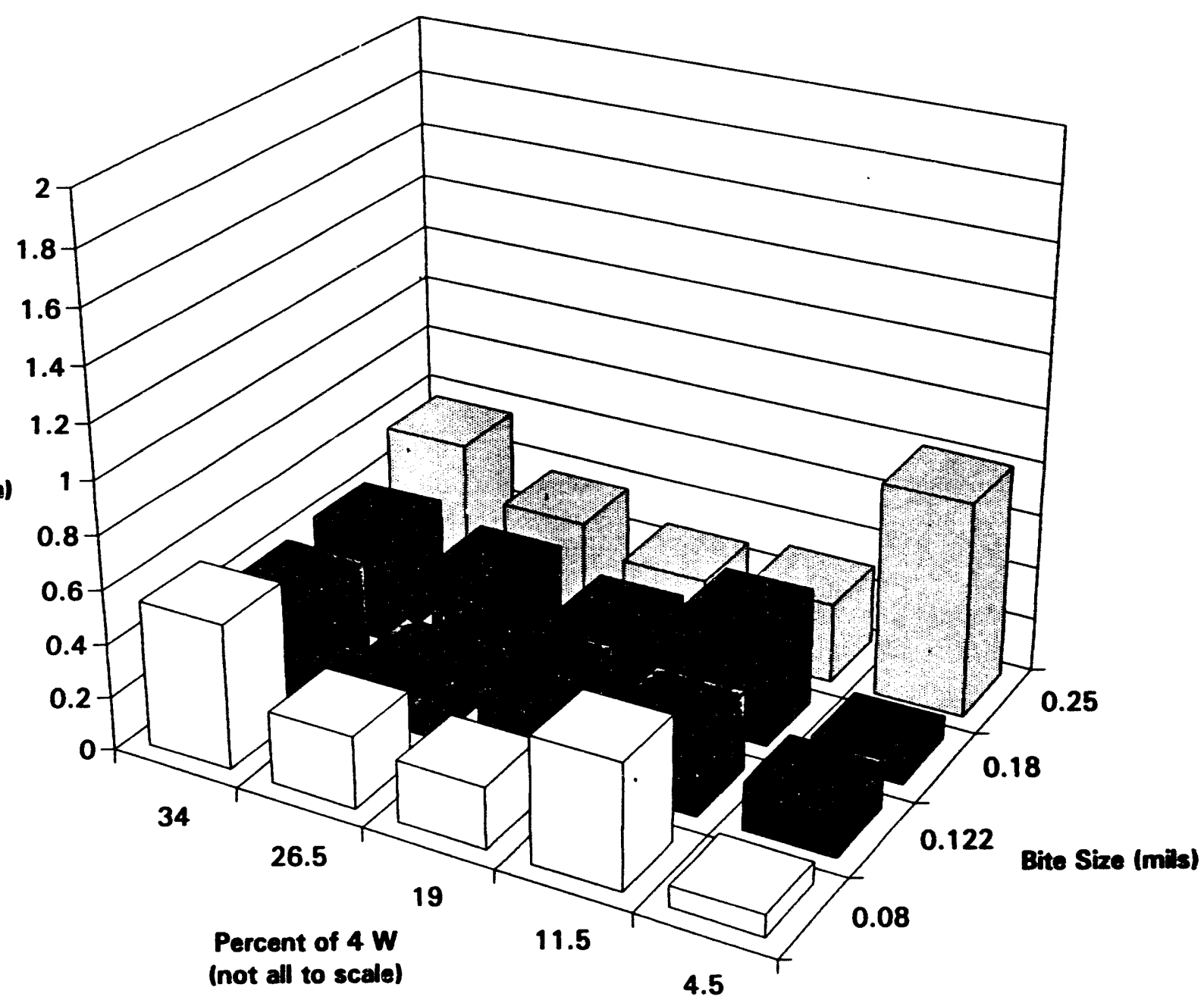




\section{Post Cycle Resistance Drift (Q-rate $=3 \mathrm{kHz}, 1000 \mathrm{ohms} / \mathrm{sq}, 11 ! 18 / 93$ )}

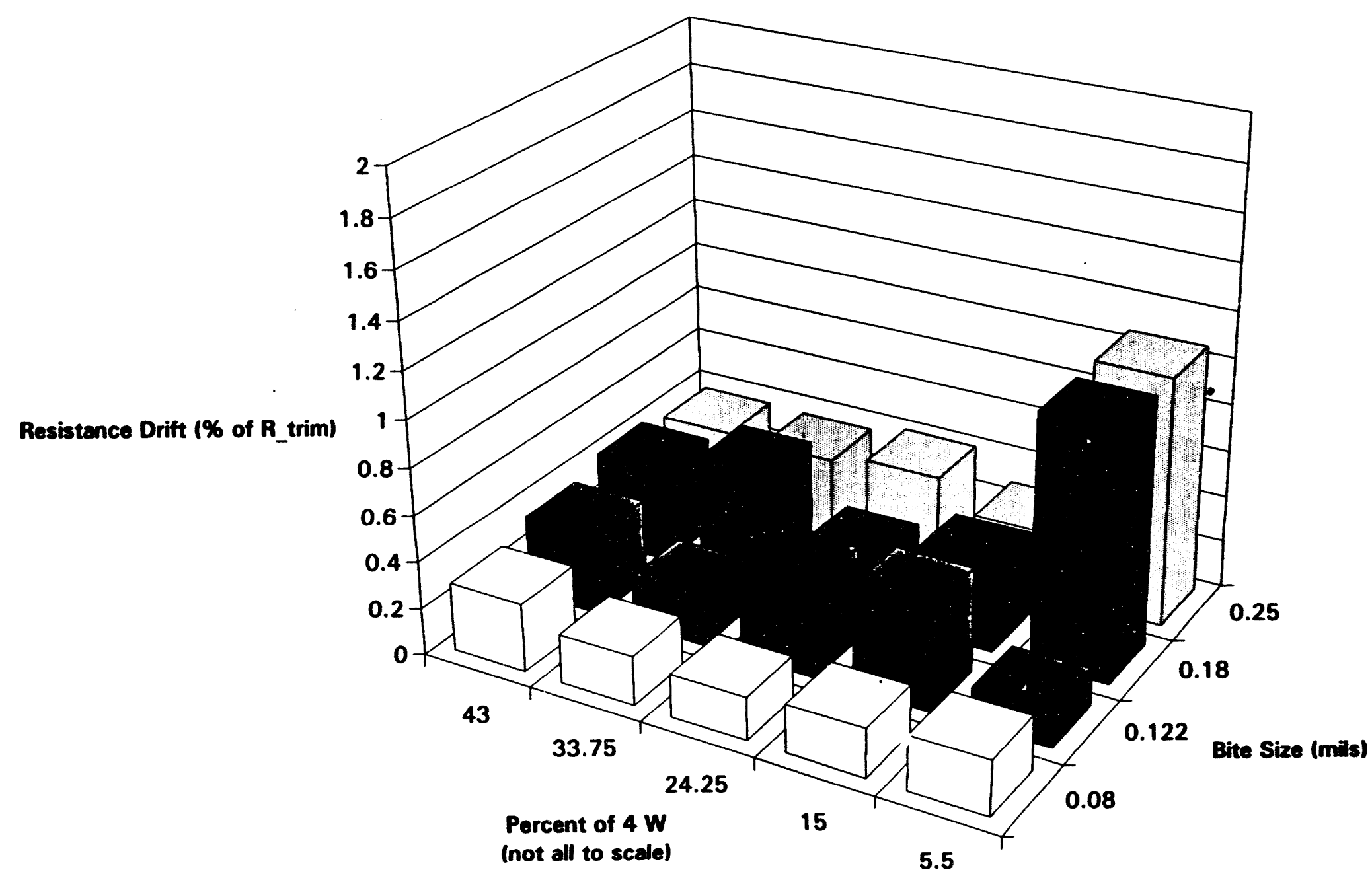


Post Cycle Resistance Drift (Q-rate $=4$ kHz, $1000 \mathrm{ohms} / \mathrm{sq}, 11 / 18 / 93$ )

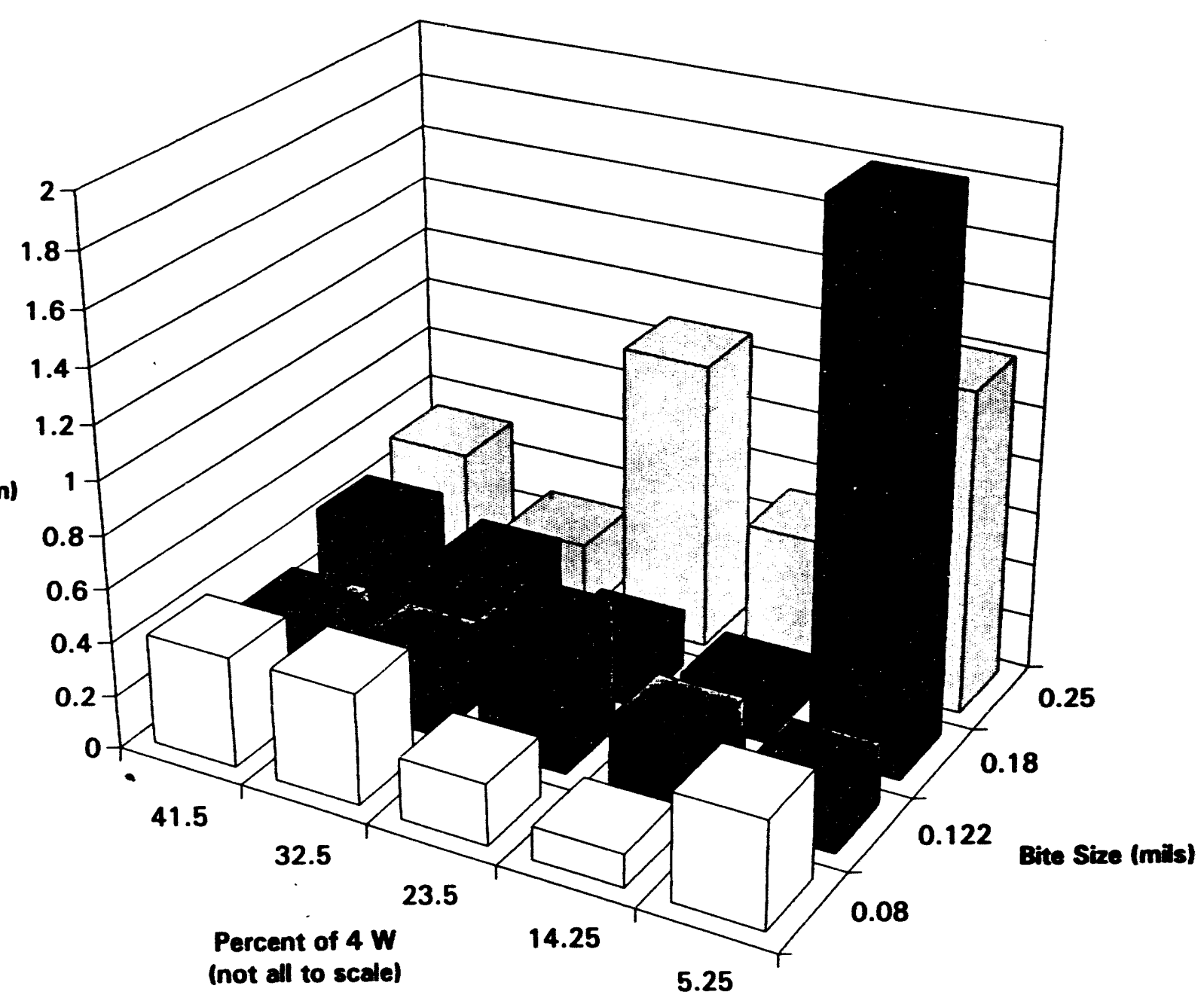


24 Hour Resistance Drift (Q-rate $=2$ kHz, 100 ohms/sq, 11/18/93)

Resistance Drift (\% of R_trim)

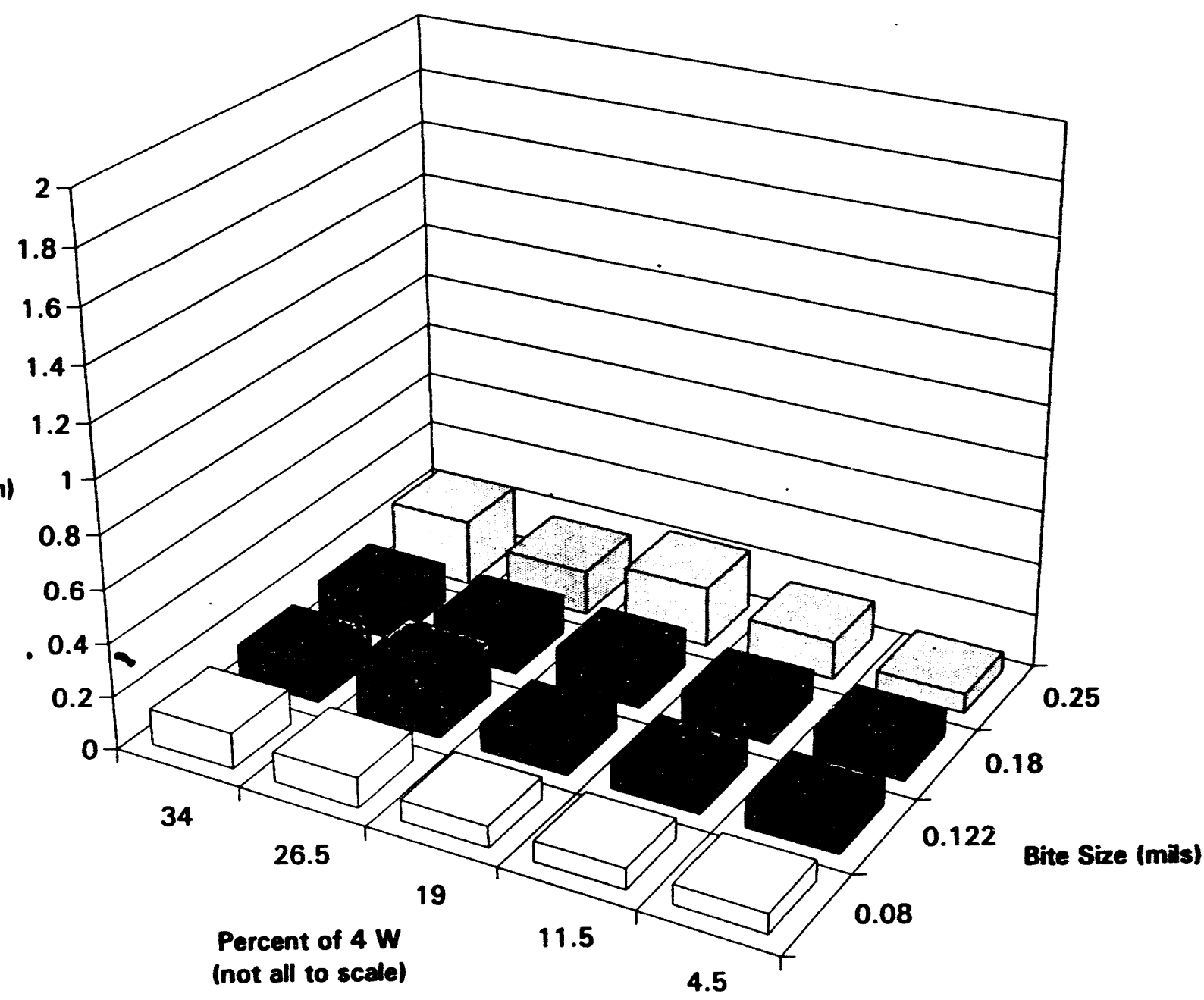




\section{Hour Resistance Drift (Q-rate $=3$ kHz, 100 ohms/sq, 11/18/93)}

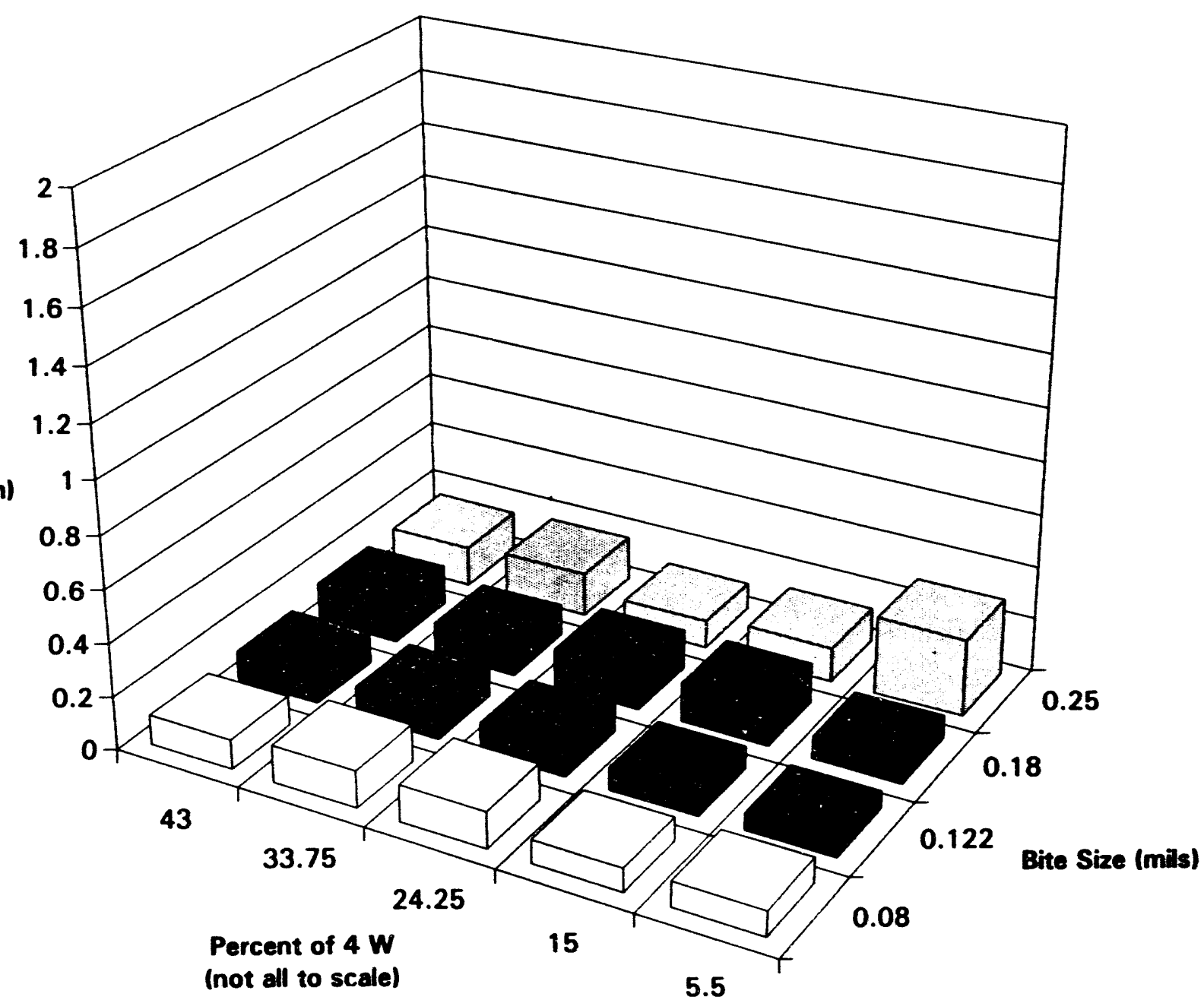


24 Hour Resistance Drift (Q-rate $=4$ kHz, $100 \mathrm{ohms} / \mathrm{sq}, 11 / 18 / 93$ )

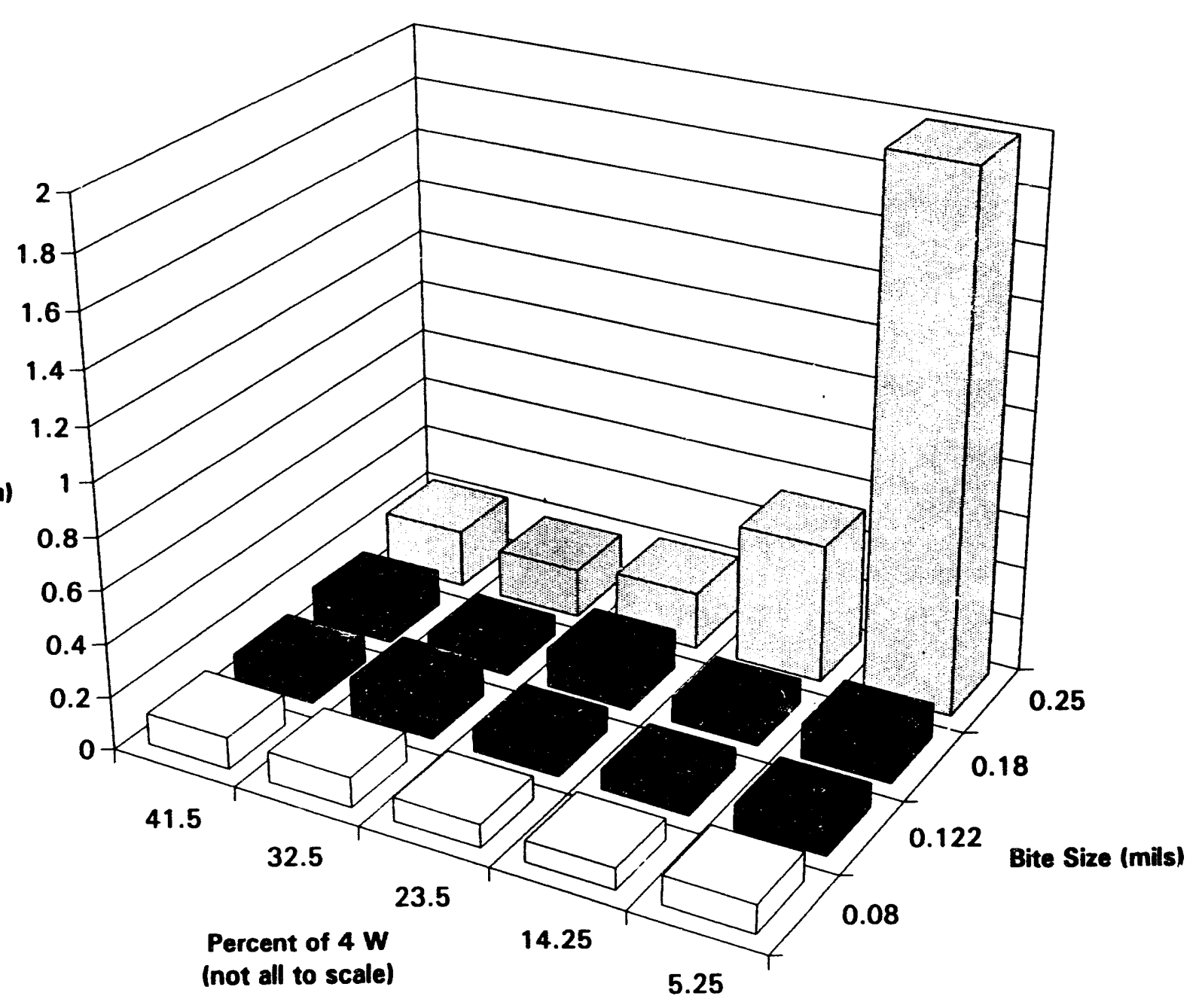


Post Cycle Resistance Drift (Q-rate $=2$ kHz, 100 ohms/sq, 11/18/93)

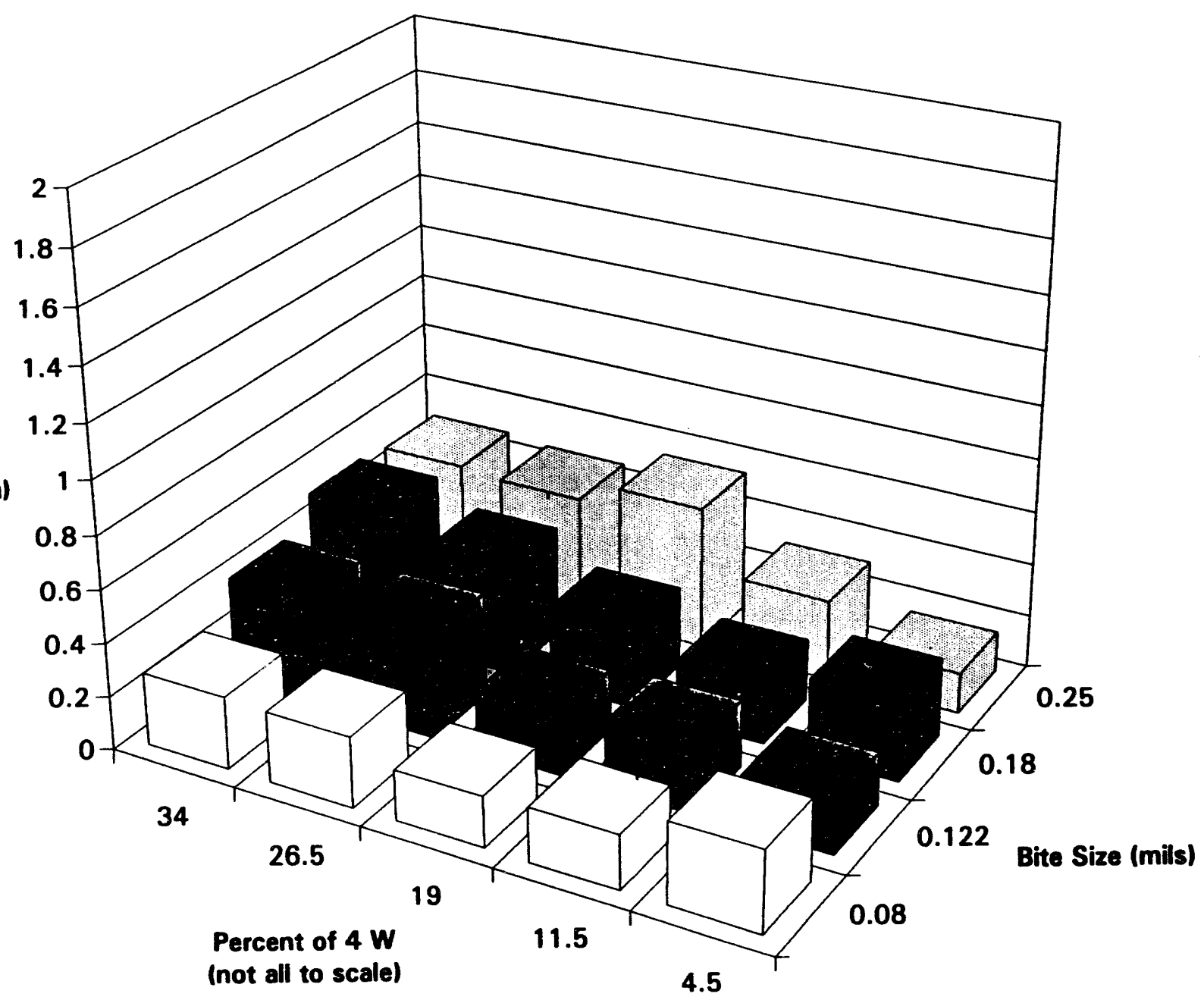


Post Cycle Resistance Drift (Q-rate $=3 \mathrm{kHz}, 100 \mathrm{ohms} / \mathrm{sq}, 11 / 18 / 93$ )

Resistance Drift (\% of R_trim)

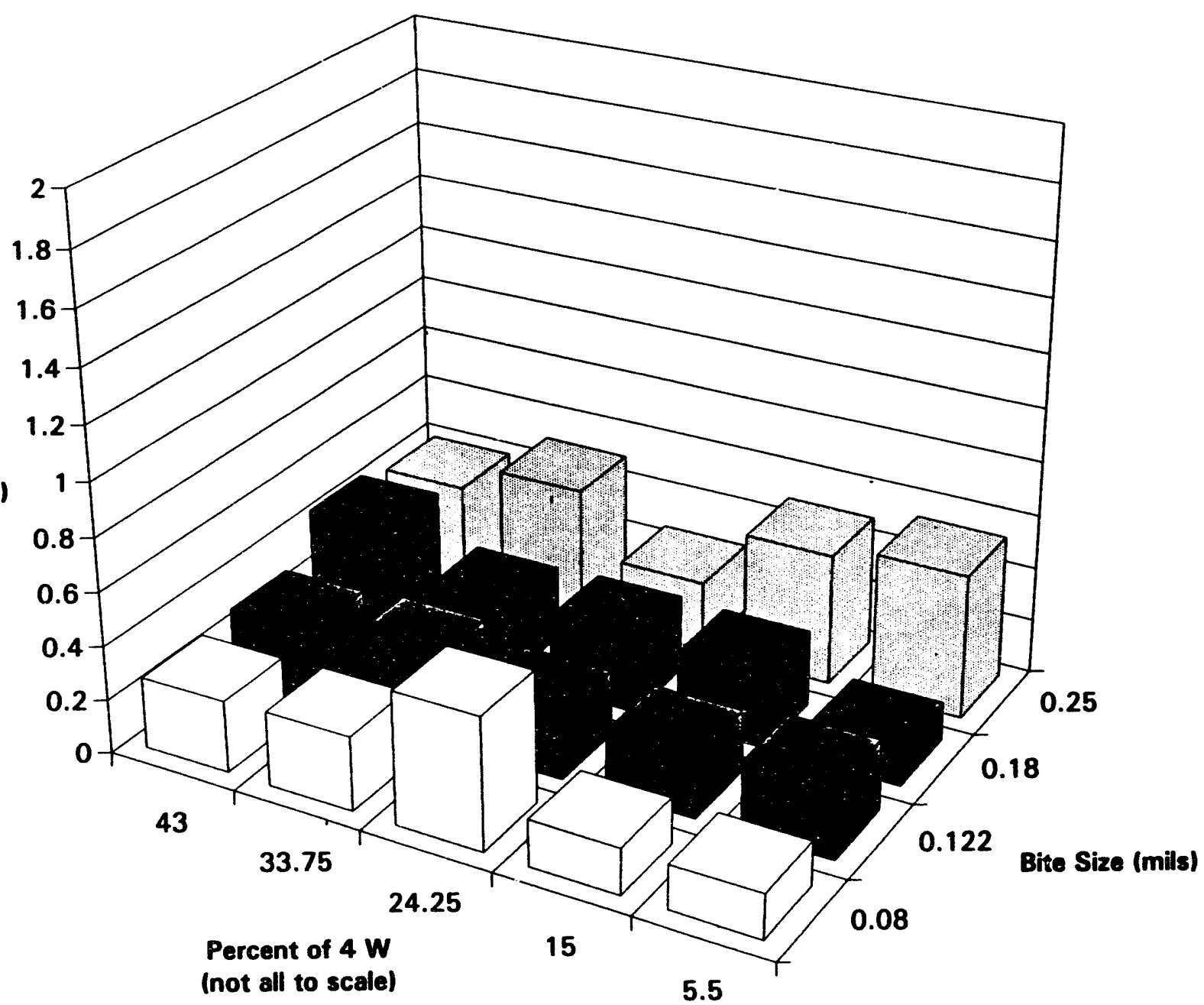




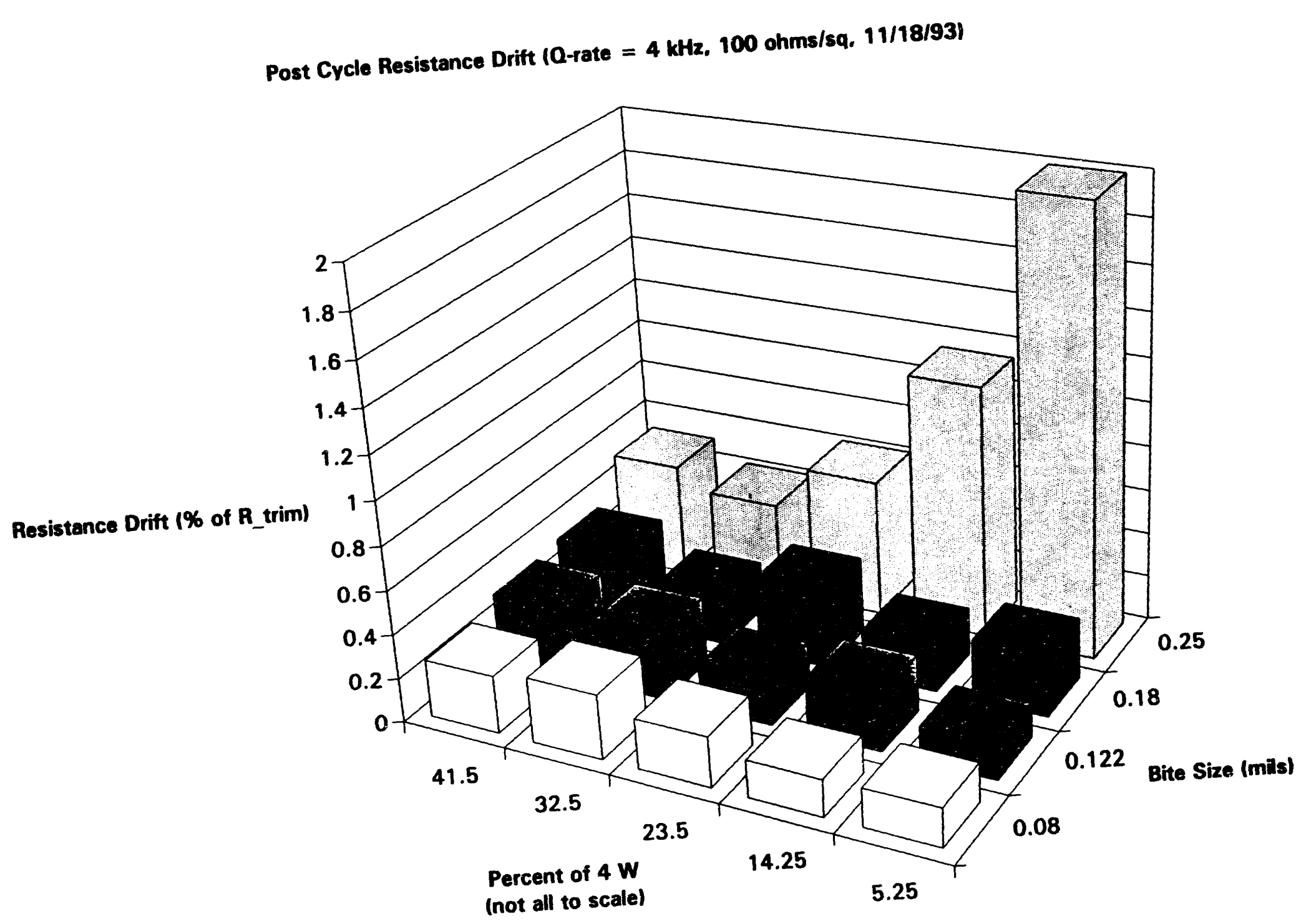


24 Hour Resistance Drift (Q-rate $=2 \mathrm{kHz}, 10 \mathrm{ohms} / \mathrm{sq}$ )

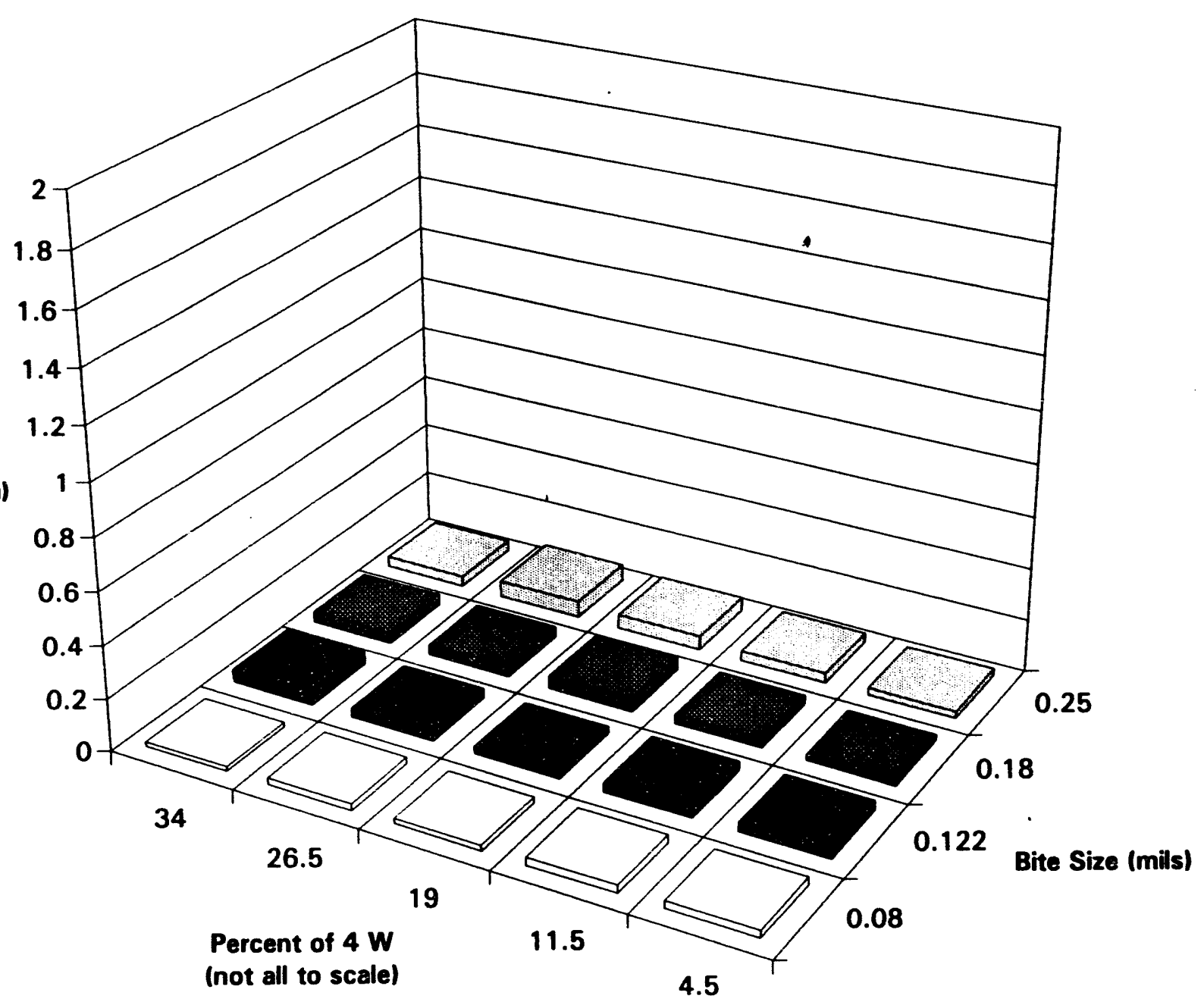


24 Hour Resistance Drift (Q-rate $=3$ kHz, $10 \mathrm{ohms} / \mathrm{sq}$ )

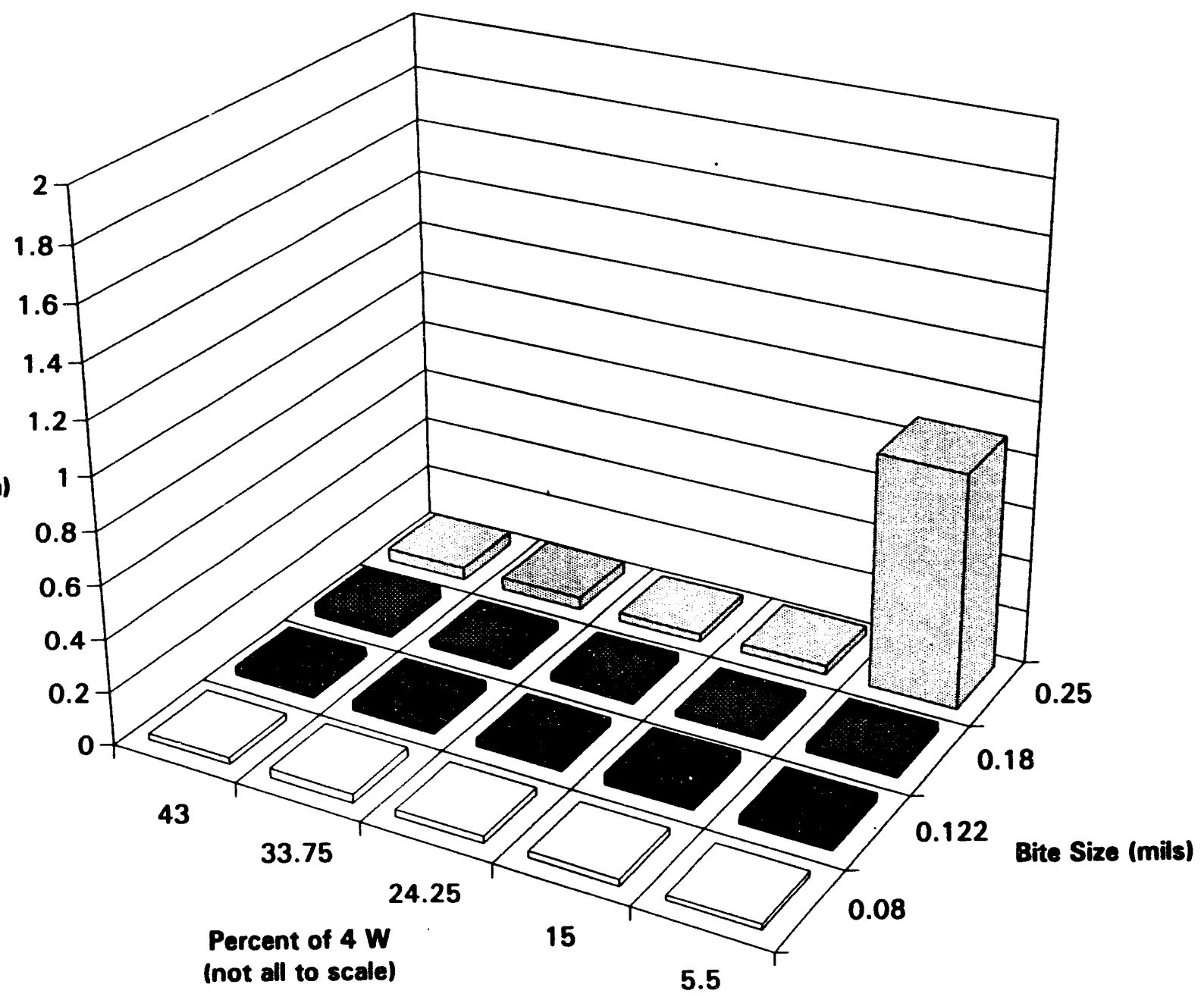


24 Hour Resistance Drift (Q-rate $=4$ kHz, $10 \mathrm{ohms} / \mathrm{sq}$ )

Resistance Drift (\% of R trim)

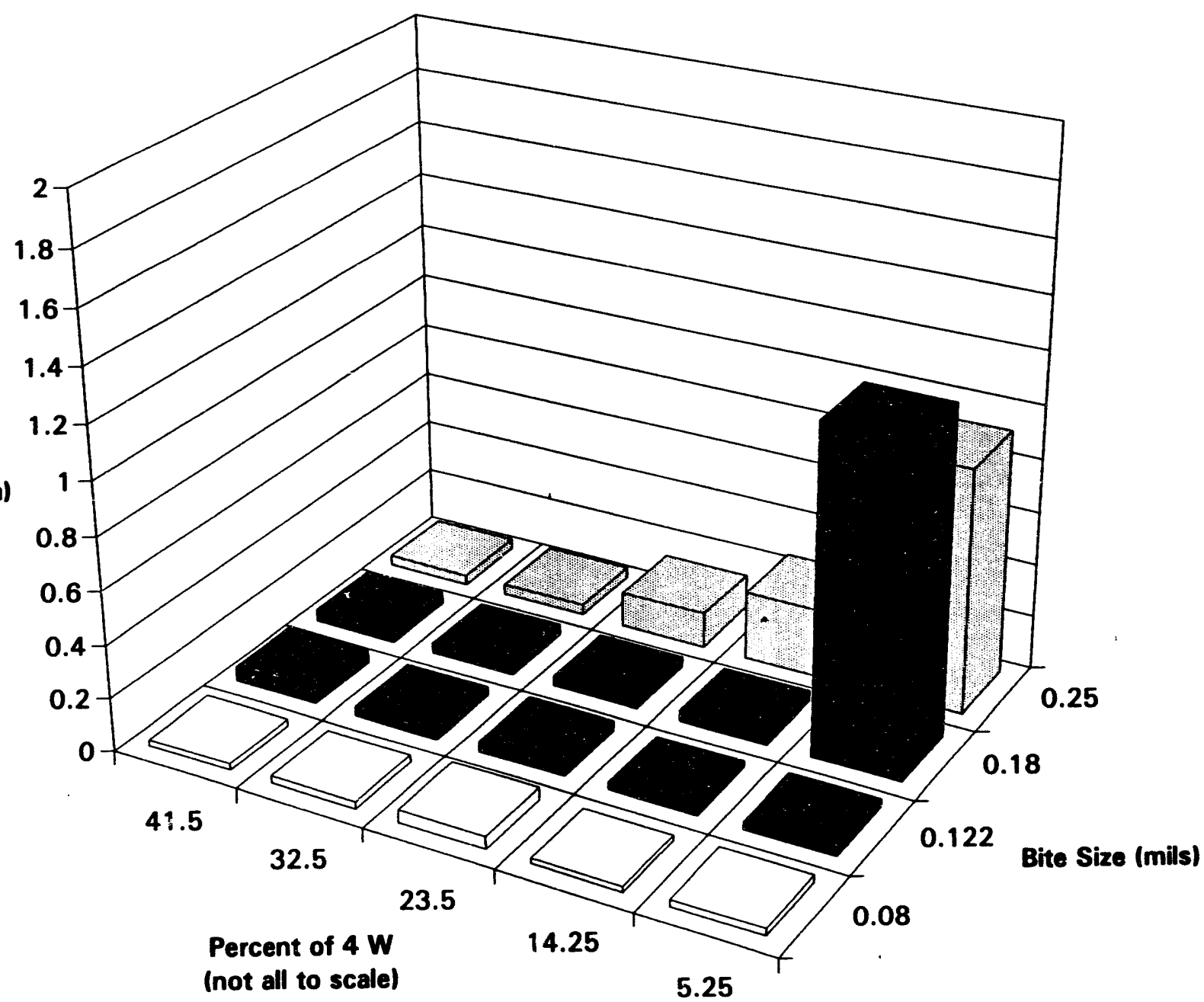


24 Hour Resistance Drift (Q-rate $=4$ kHz, $10 \mathrm{ohms} / \mathrm{sq}$, questionable data points removed)

Resistance Drift (\% of R_trim)

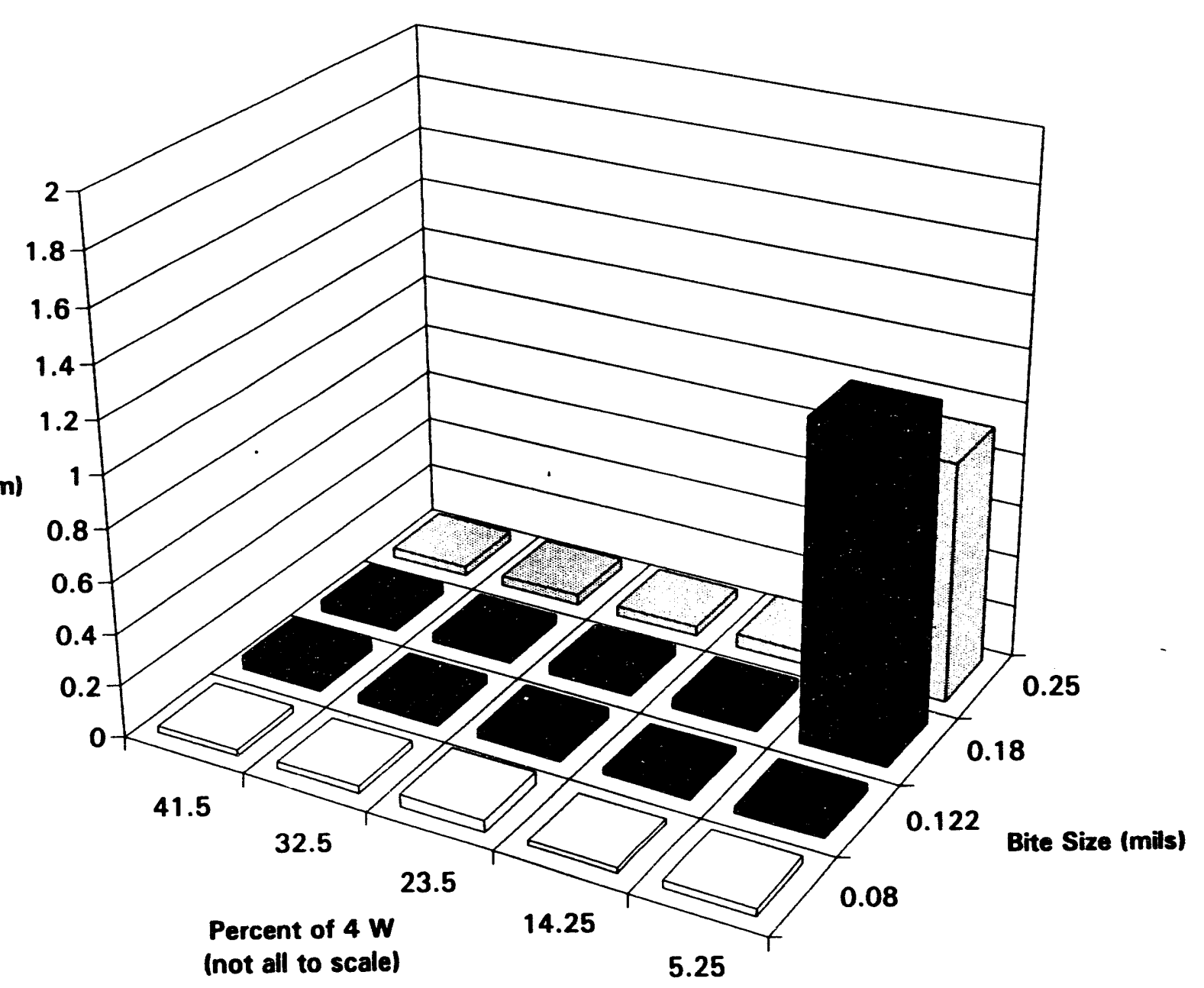


Post Cycle Resistance Drift (Q-rate $=2$ kHz, $10 \mathrm{ohms} / \mathrm{sq}$ )

Resistance Drift $1 \%$ of R_trim)

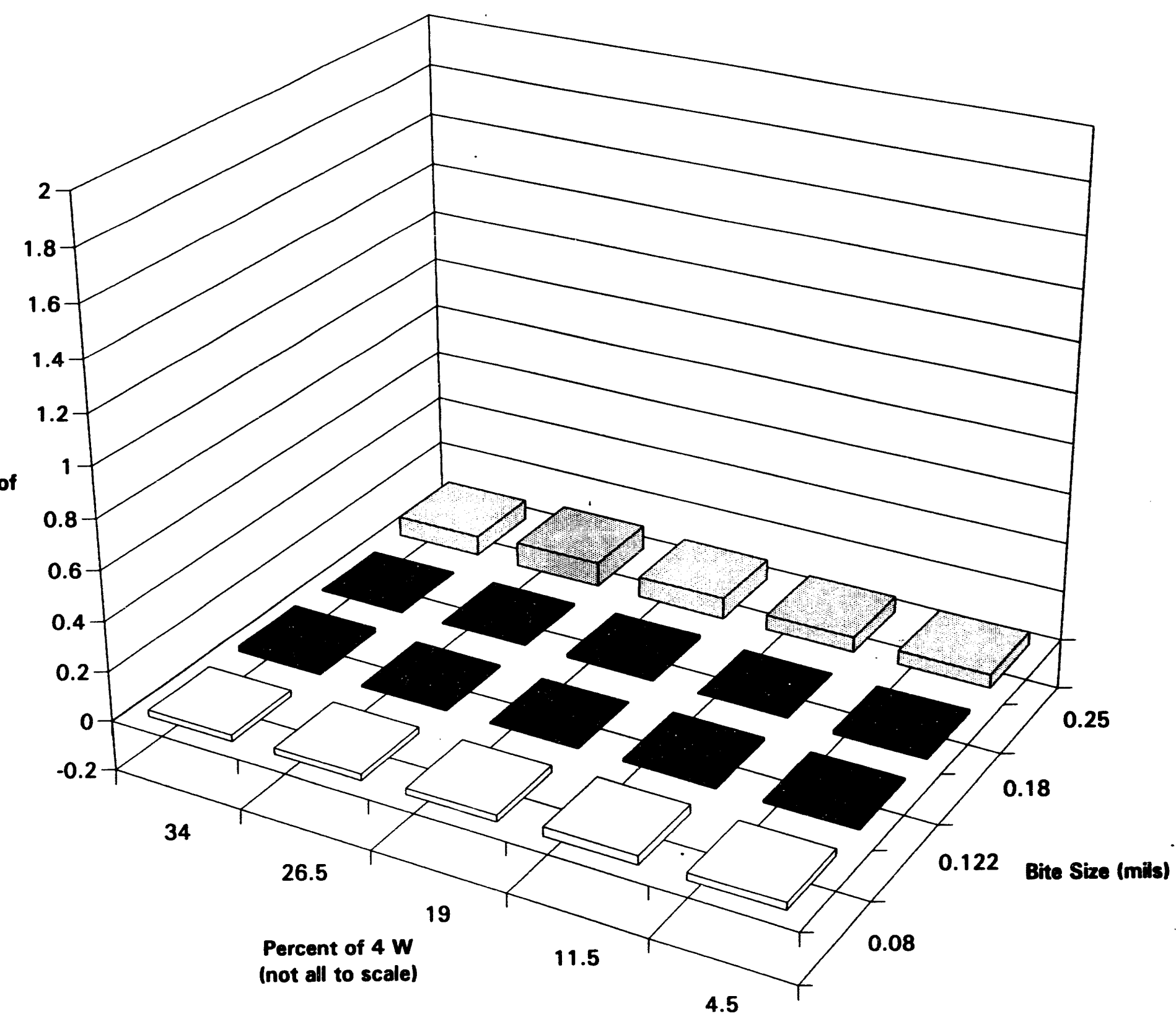




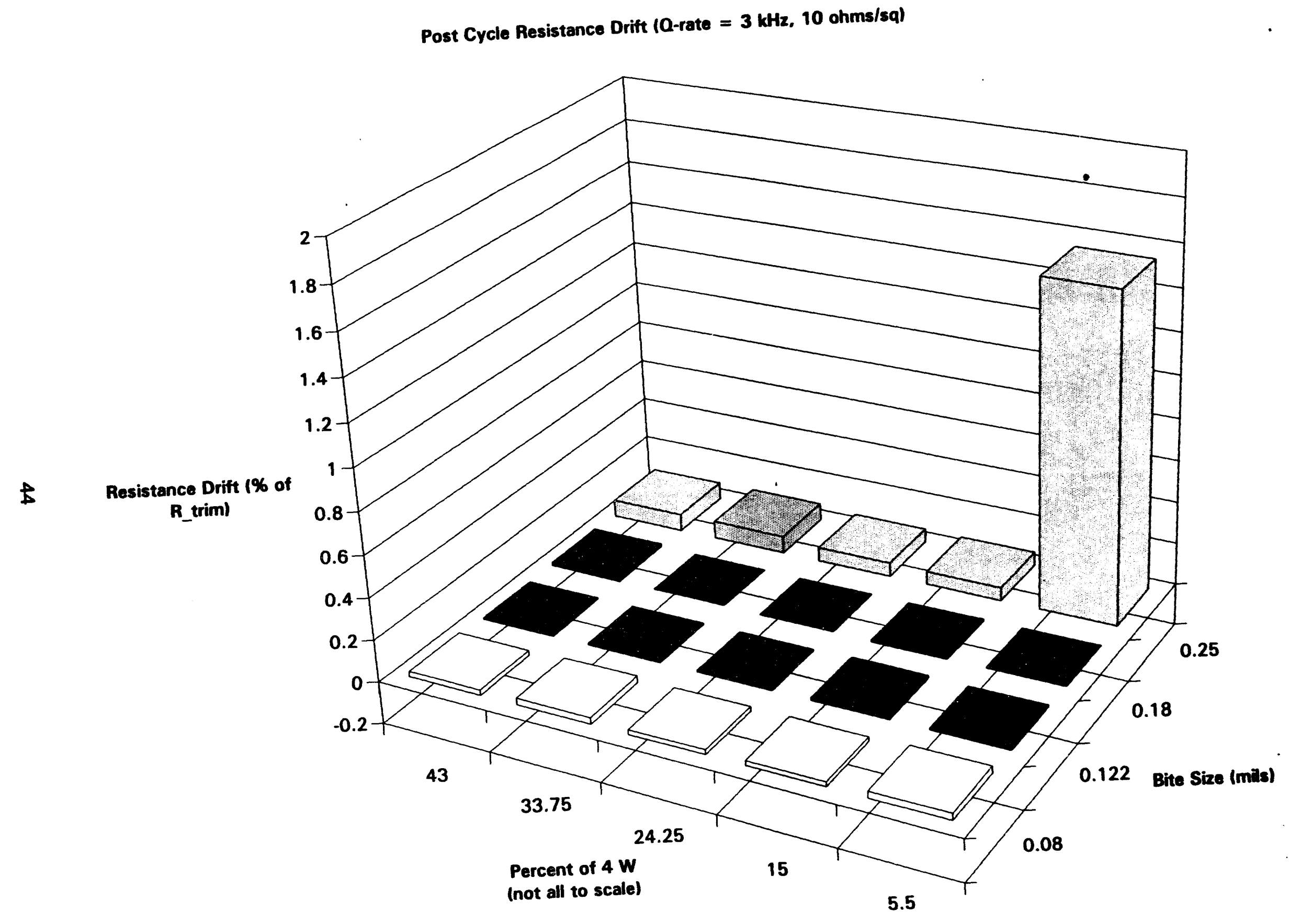




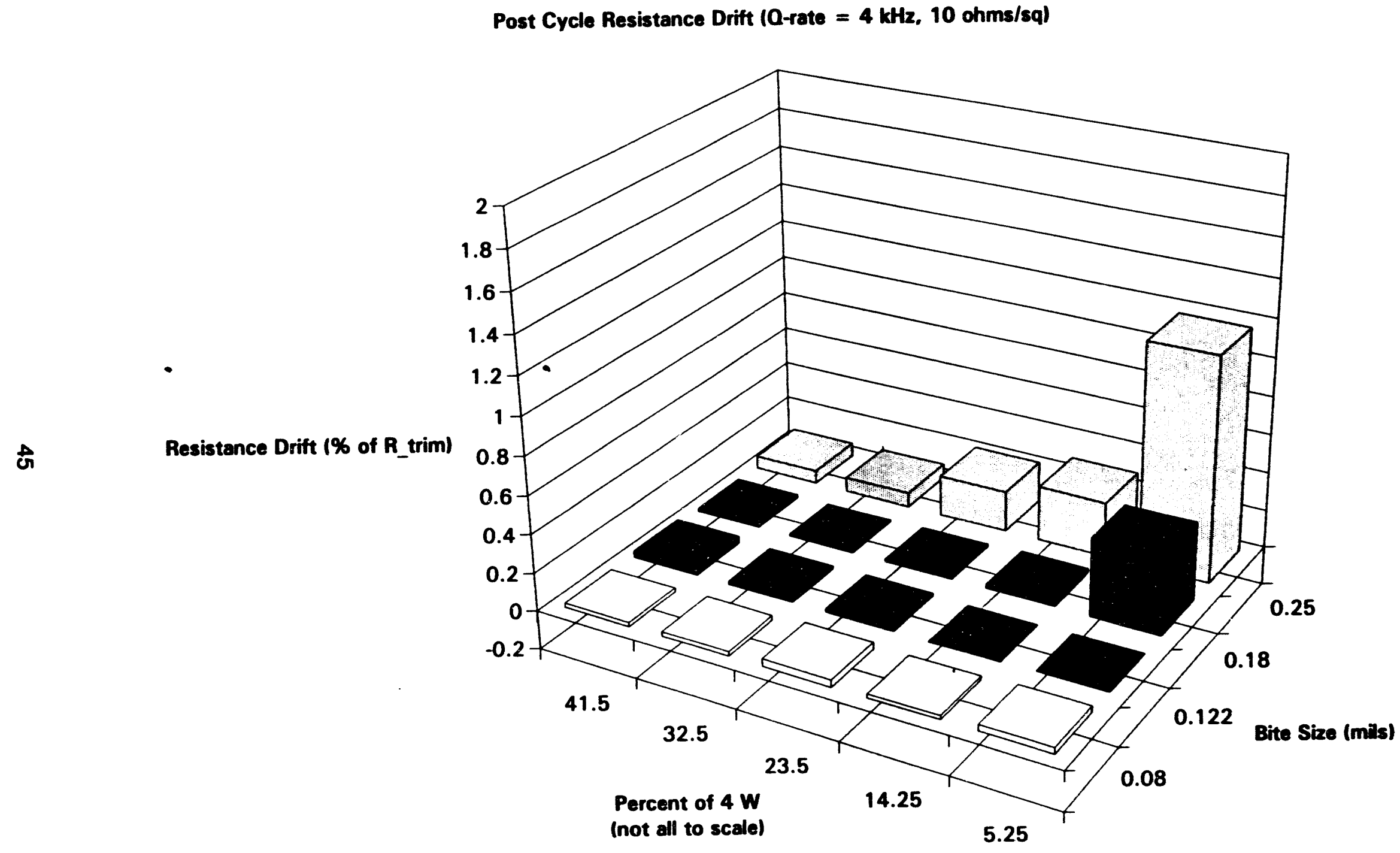




\section{Post Cycle Resistance Drift, Magnitude (Q-rate $=2 \mathrm{kHz}, 10 \mathrm{ohms} / \mathrm{sq}$ )}

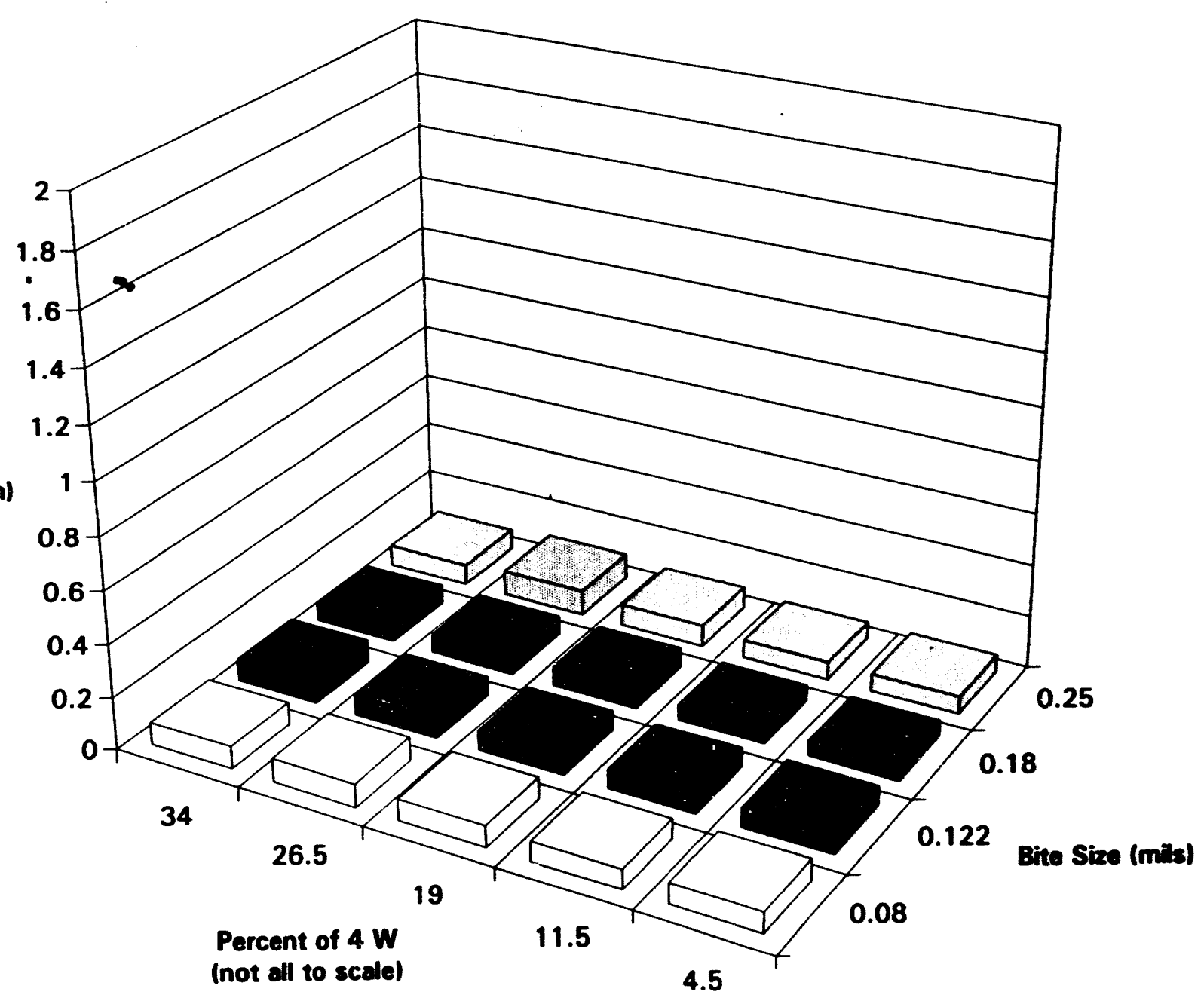




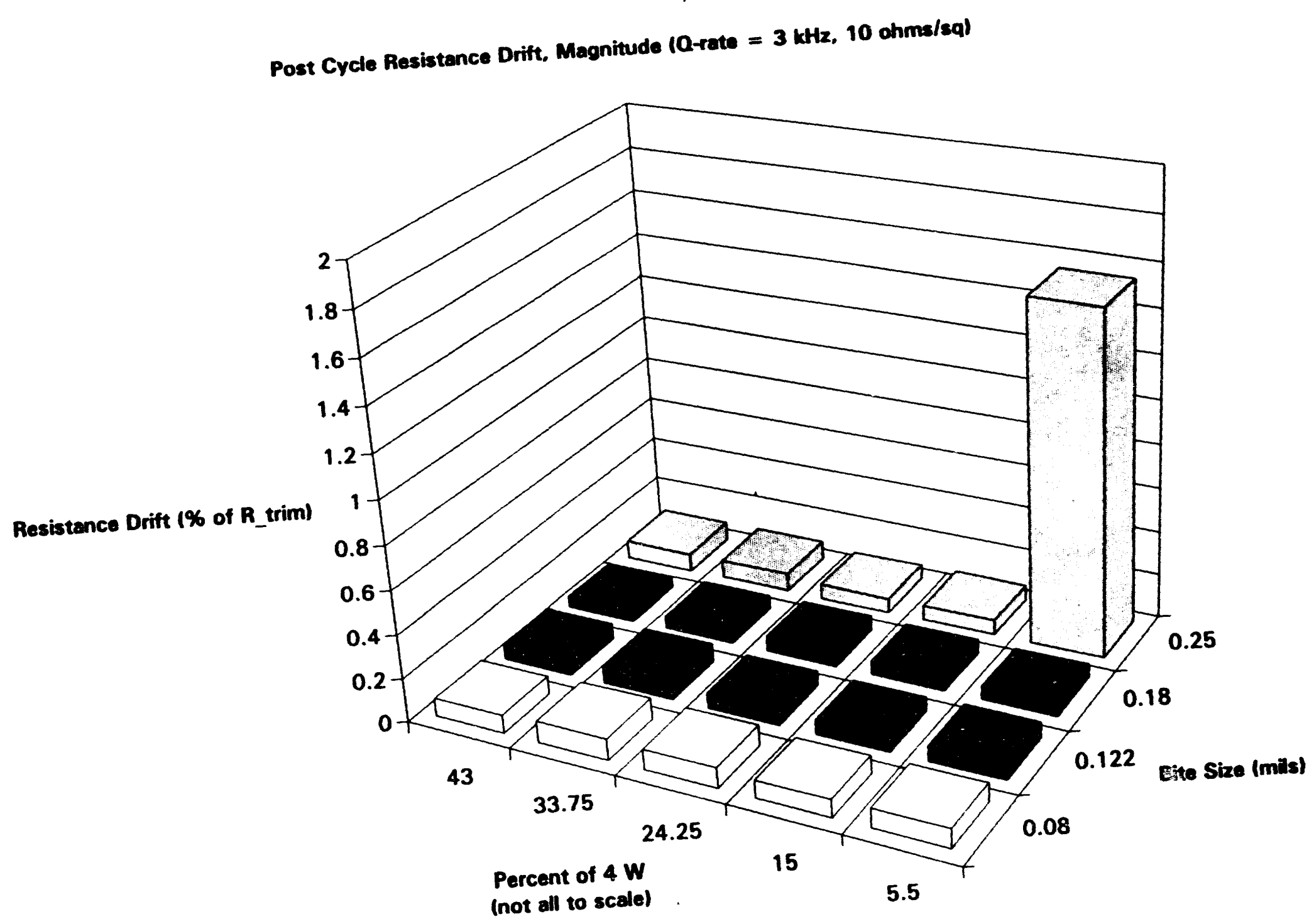




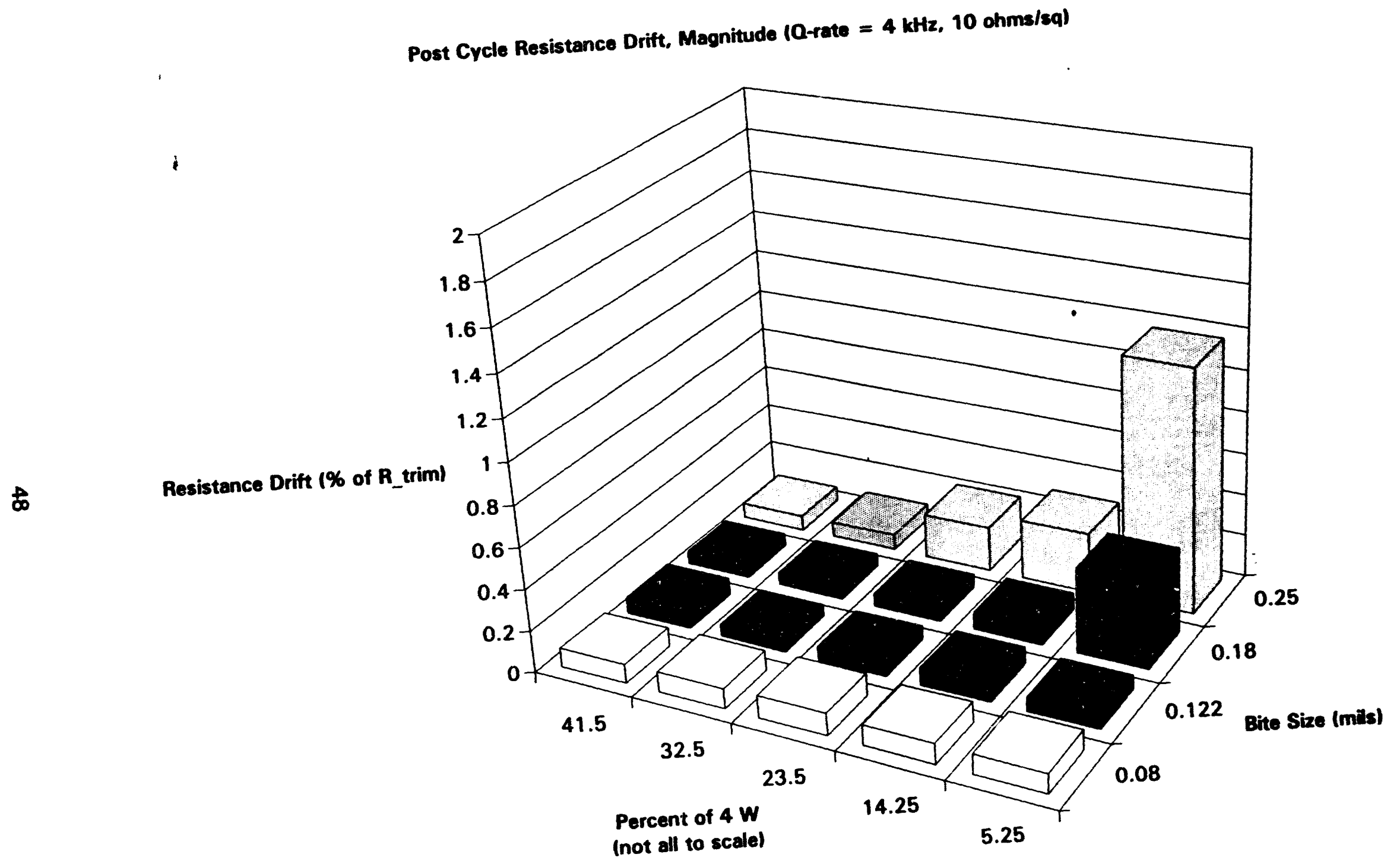


Post Cycle Resistance Drift, Magnitude (Q-rate $=4 \mathrm{kHz}, 10 \mathrm{ohms} / \mathrm{sq}$, questional data points removed)

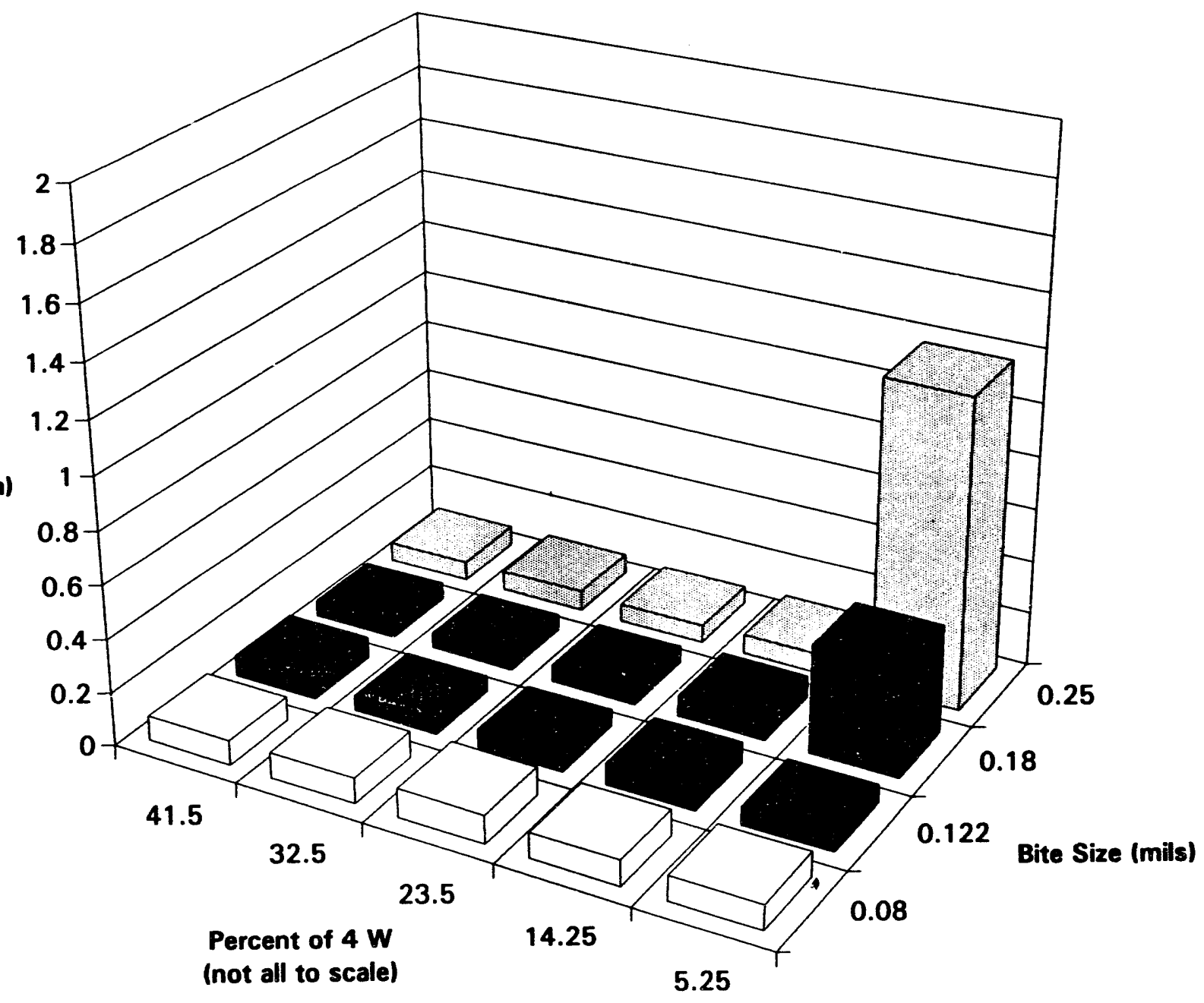


Histogram, nominal zup +20 mils (thick film, 10k ohms/sq)

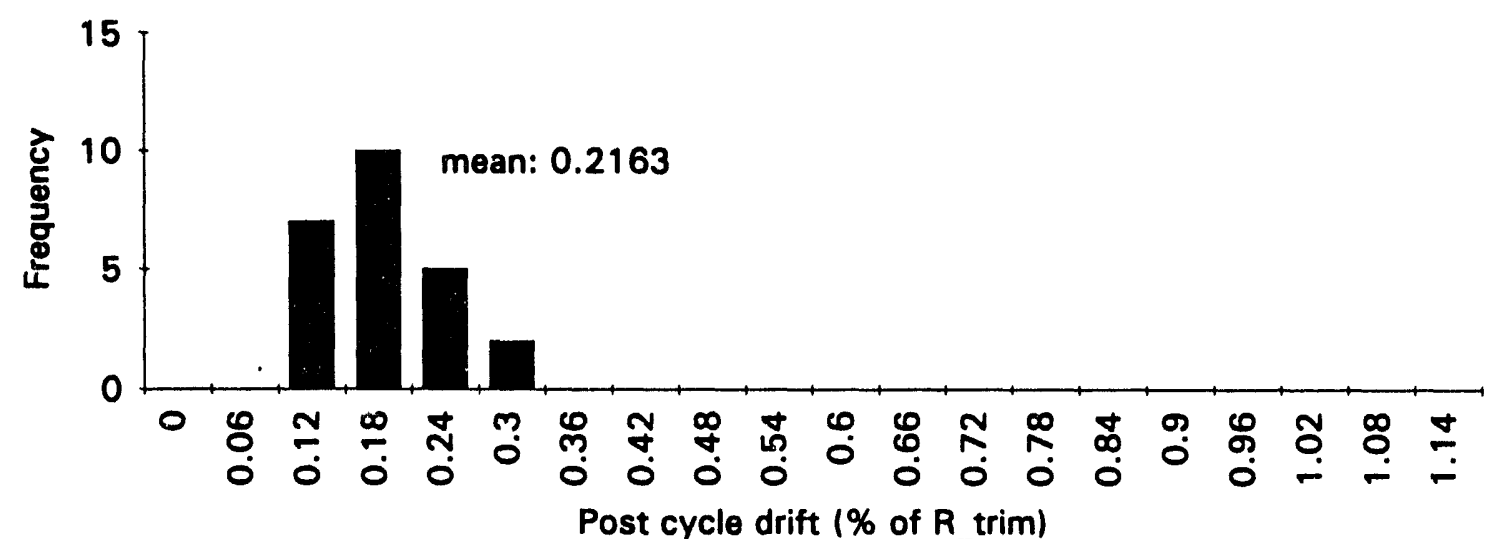

Histogram, nominal zup +0 mils (thick film, 10k ohms/sq)

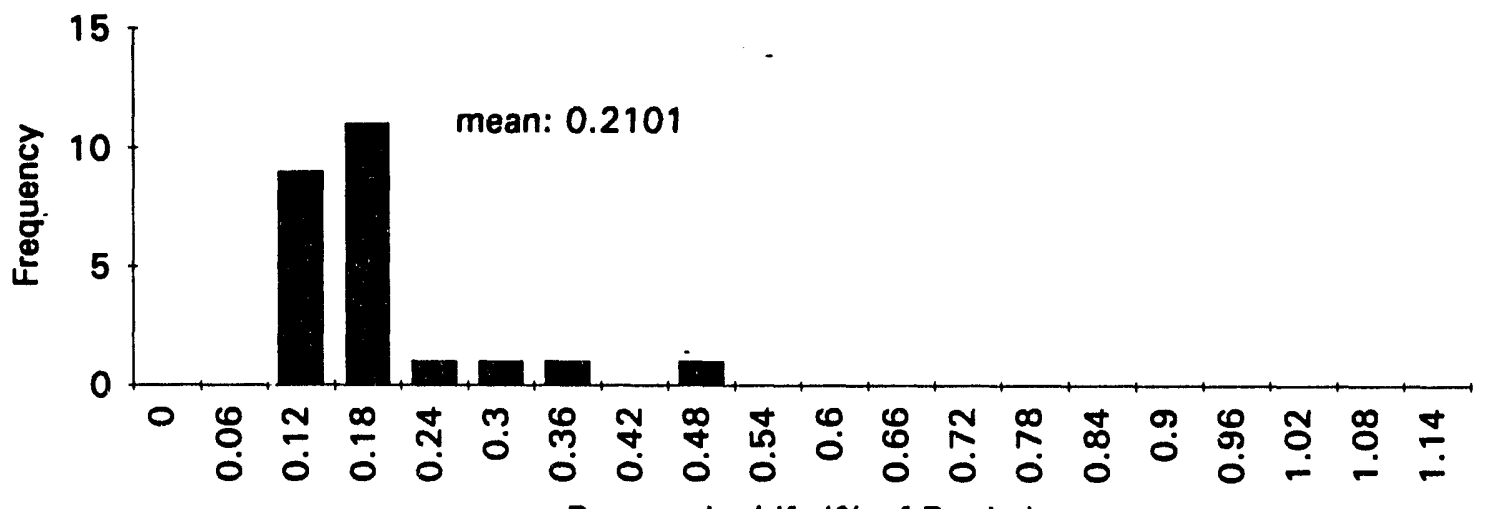

Post cycle drift (\% of R_trim)

Histogram, nominal zup - 20 mils (thick film, 10k ohms/sq)

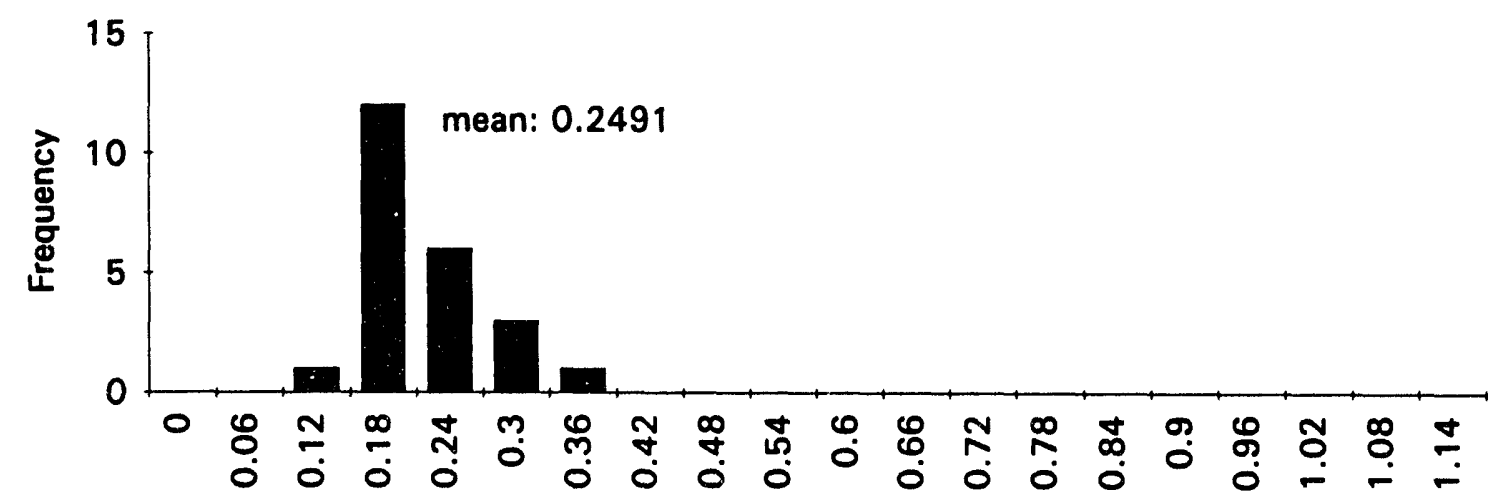

Post cycle drift (\% of R_trim) 


\begin{tabular}{|c|c|c|c|c|c|c|c|c|c|c|c|c|c|c|c|c|c|c|c|}
\hline & & & & 0 & \begin{tabular}{l|l}
0 & 10
\end{tabular} & চান & 100 & 10 & 010 & 50 & 00 & 10 & 00 & 1010 & & & & 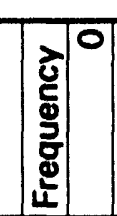 & 00 \\
\hline & & & & $\frac{1}{0}$ & 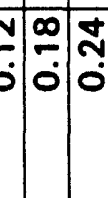 & $\underset{0}{*}$ & & & $\begin{array}{l} \\
\\
0 \\
0 \\
0\end{array}$ & $\dot{0}$ & \begin{tabular}{l|l} 
& $\infty$ \\
0 & 0 \\
\end{tabular} & 志 & 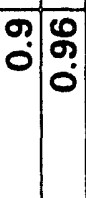 & $\stackrel{\alpha}{\alpha}$ & $\stackrel{5}{=}$ & & & (5) & $0_{0}^{0} \stackrel{2}{0}$ \\
\hline & & & 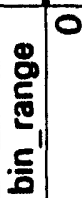 & 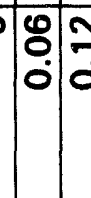 & 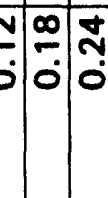 & $\begin{array}{c} \pm \\
0 \\
0\end{array}$ & 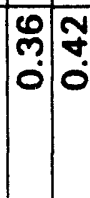 & & $\begin{array}{c}0 \\
0 \\
0 \\
0\end{array}$ & $:$ & & 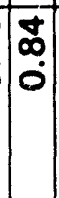 & व̊: & $\stackrel{0}{0}$ & $\stackrel{0}{ \pm}$ & & & & \\
\hline & & & 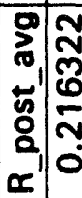 & & & & & & & & & & & & & & & 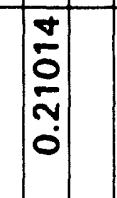 & \\
\hline & & & 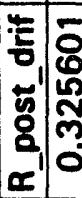 & 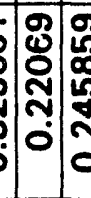 & 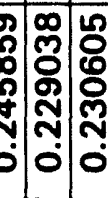 & 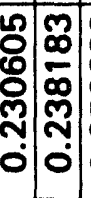 & 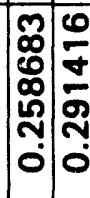 & 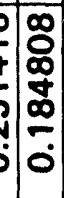 & 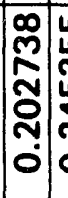 & 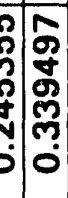 & & 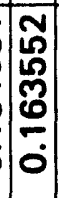 & 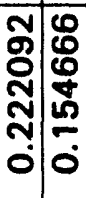 & 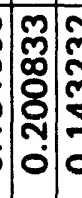 & 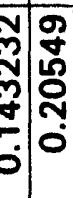 & 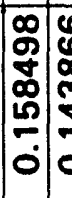 & 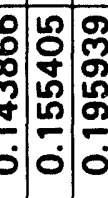 & 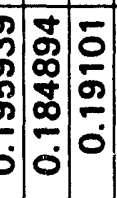 & 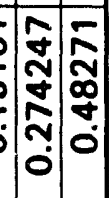 \\
\hline & & & 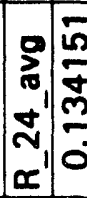 & & & & & & & & & & & & & & & $\mid$\begin{tabular}{c}
$\hat{n}$ \\
$\frac{m}{m}$ \\
\hdashline 0 \\
0
\end{tabular} & \\
\hline & & & 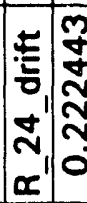 & 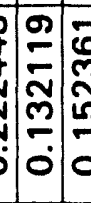 & 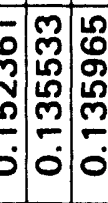 & 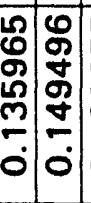 & 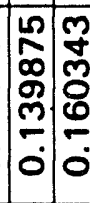 & & 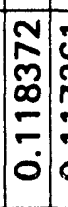 & 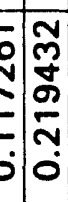 & & \begin{tabular}{|c}
9 \\
$\tilde{D}$ \\
0 \\
\hdashline \\
0
\end{tabular} & 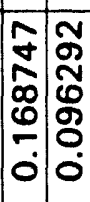 & 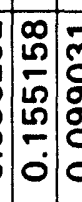 & 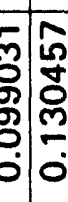 & 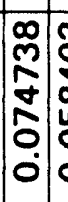 & 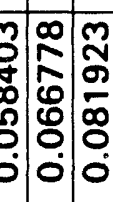 & 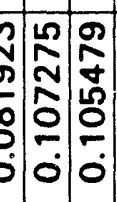 & 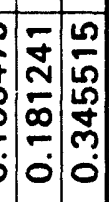 \\
\hline & & & 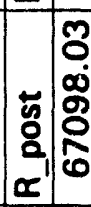 & 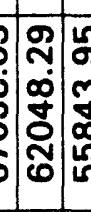 & 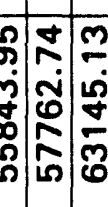 & 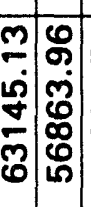 & 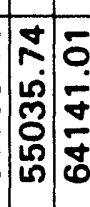 & 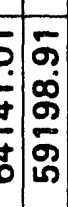 & 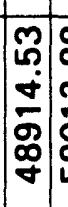 & 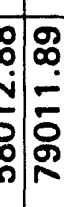 & & 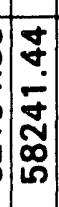 & 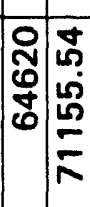 & {$\left[\begin{array}{l}1 \\
\hdashline \\
0 \\
0 \\
0 \\
0\end{array}\right.$} & 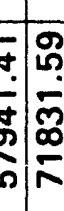 & 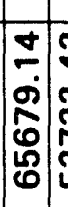 & & : & 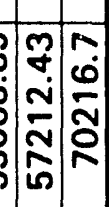 \\
\hline $\begin{array}{c}\sum_{\bar{L}} \\
\mid \\
\vdots\end{array}$ & & & 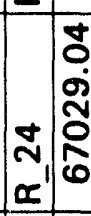 & 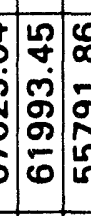 & 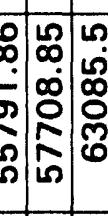 & 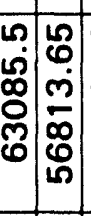 & 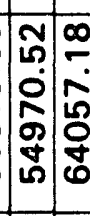 & 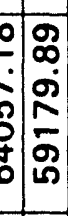 & 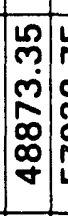 & 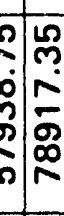 & 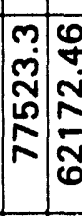 & 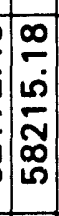 & 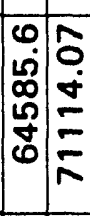 & 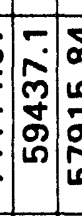 & 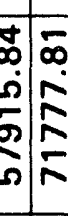 & 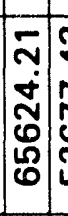 & 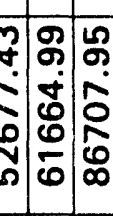 & 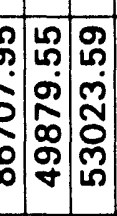 & 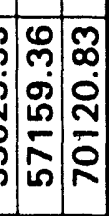 \\
\hline $\bar{T}=$ & & & & & & হిন & নিহ & & Di: & $\sqrt{2}$ & & 요 & হিন্ & సিঙ & $\sqrt{\mathrm{N}}$ & & ลิনহ & & 100 \\
\hline 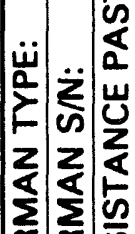 & 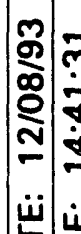 & 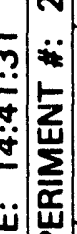 & 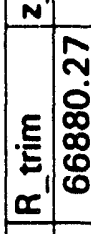 & 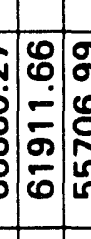 & 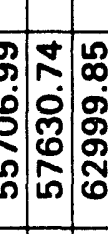 & 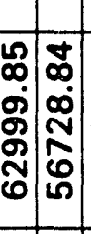 & 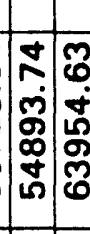 & & 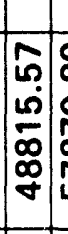 & 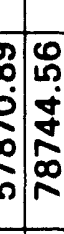 & 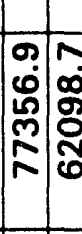 & 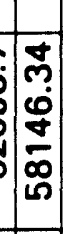 & 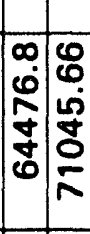 & 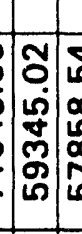 & 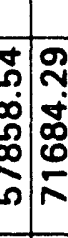 & 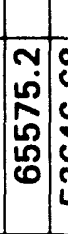 & 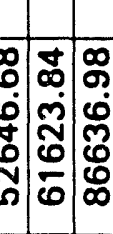 & 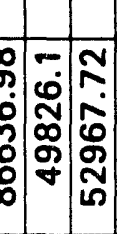 & 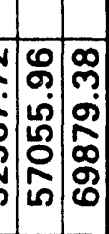 \\
\hline & & & & & $\frac{\alpha}{\infty}$ & $\overline{\boldsymbol{\infty}} \boldsymbol{\tilde { \omega }}$ & $m_{\infty}^{\infty}$ & & & & & & & & & & $m$ & 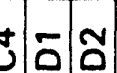 & $m i$ \\
\hline
\end{tabular}




\section{LASR0238.CSV}

\begin{tabular}{|c|c|c|c|c|c|c|c|c|c|c|c|}
\hline E1 & 55856.01 & 0 & 55917.71 & 55959.39 & 0.110475 & & 0.185097 & & & 0.18 & 11 \\
\hline E2 & 59756.26 & 0 & 59815.55 & 59876.98 & 0.099221 & & 0.20202 & & & 0.24 & 1 \\
\hline E3 & 50232.95 & 0 & 50288.81 & 50338.11 & 0.111194 & & 0.209348 & & & 0.3 & 1 \\
\hline E4 & 59836.09 & 0 & 59965.77 & 60024.54 & 0.216735 & & 0.314951 & & & 0.36 & 1 \\
\hline F1 & 62473.25 & 0 & 62532.31 & 62597.77 & 0.094542 & & 0.199317 & & & 0.42 & 0 \\
\hline $\mathbf{F 2}$ & 62878.94 & 0 & 62932.29 & 62997.73 & 0.084846 & & 0.18891 & & & 0.48 & 1 \\
\hline F3 & 49231.07 & 0 & 49300.43 & 49338.16 & 0.140887 & & 0.217511 & & & 0.54 & 0 \\
\hline F4 & 57901.47 & 0 & 57955.98 & 58001.53 & 0.094158 & & 0.172824 & & & 0.6 & 0 \\
\hline D1 & 53403.51 & 0 & 53445.45 & 53488.14 & 0.078549 & & 0.158475 & & & 0.66 & 0 \\
\hline D2 & 57070.95 & 0 & 57102.8 & 57142.48 & 0.055814 & & 0.125336 & & & 0.72 & 0 \\
\hline D3 & 66909.02 & 0 & 66952.18 & 67021.99 & 0.064503 & & 0.168829 & & & 0.78 & 0 \\
\hline D4 & 74239.5 & 0 & 74296.47 & 74363.35 & 0.076738 & & 0.166829 & & & 0.84 & 0 \\
\hline E1 & 58124.16 & 0 & 58179.59 & 58206.05 & 0.095359 & & 0.140884 & & & 0.9 & 0 \\
\hline E2 & 62788.65 & 0 & 62871.48 & 62910.66 & 0.131922 & & 0.194314 & & & 0.96 & 0 \\
\hline E3 & 59017.35 & 0 & 59111.96 & 59143.03 & 0.16032 & & 0.212961 & & & 1.02 & 0 \\
\hline E4 & 64881.12 & 0 & 65098.61 & 65135.24 & 0.335223 & & 0.391669 & & & 1.08 & 0 \\
\hline F1 & 67315.01 & 0 & 67393.33 & 67427.45 & 0.116347 & & 0.167035 & & & 1.14 & 0 \\
\hline F2 & 63759.53 & 0 & 63817.68 & 63849.01 & 0.091205 & & 0.140333 & & & & \\
\hline F3 & 57137.04 & 0 & 57189.5 & 57218.57 & 0.091814 & & $0.1,42687$ & & & & \\
\hline $\mathbf{F 4}$ & 65766.99 & 0 & 65874.33 & 65905.87 & 0.163215 & & 0.211162 & & & & \\
\hline G1 & 53454.79 & -20 & 53537.98 & 53625 & 0.155632 & 0.155621 & 0.318425 & 0.24909 & & Bin & Frequency \\
\hline $\mathbf{G 2}$ & 67048.09 & -20 & 67193.84 & 67261.3 & 0.21738 & & 0.317995 & & & 0 & 0 \\
\hline G3 & 59421.53 & -20 & 59501.76 & 59571.15 & 0.135035 & & 0.251803 & & & 0.06 & 0 \\
\hline G4 & 58083.05 & -20 & 58183.21 & 58237.38 & 0.172451 & & 0.265712 & & & 0.12 & 1 \\
\hline $\mathrm{H1}$ & 52221.82 & -20 & 52281.04 & 52343.26 & 0.113406 & & 0.232556 & & & 0.18 & 12 \\
\hline $\mathrm{H} 2$ & 62466.86 & -20 & 62547.19 & 62603.58 & 0.128602 & & 0.218867 & & & 0.24 & 6 \\
\hline $\mathrm{H3}$ & 63238.34 & -20 & 63347.39 & 63423.13 & 0.172445 & & 0.292209 & & & 0.3 & 3 \\
\hline $\mathrm{H4}$ & 58082.64 & -20 & 58163.57 & 58210.35 & 0.139339 & & 0.219883 & & & 0.36 & 1 \\
\hline 11 & 48278.2 & -20 & 48377.09 & 41295.25 & 0.204846 & & & & & 0.42 & 0 \\
\hline 12 & 58408.73 & -20 & 58478.63 & 58537.21 & 0.119662 & & 0.219964 & & & 0.48 & 0 \\
\hline 13 & 63507.07 & -20 & 63613.04 & 63704.7 & 0.166861 & & 0.311192 & & & 0.54 & 0 \\
\hline 14 & 69209.92 & -20 & 69304.67 & 69405.48 & 0.136908 & & 0.282561 & & & 0.6 & 0 \\
\hline $\mathbf{G 1}$ & 54894.75 & -20 & 54972.61 & 55017.21 & 0.141835 & & 0.223077 & & & 0.66 & 0 \\
\hline G2 & 65066.74 & -20 & 65187.88 & 65231.33 & 0.18617 & & 0.252944 & & & 0.72 & 0 \\
\hline G3 & 66843.63 & -20 & 66935.52 & 66987.22 & 0.137471 & & 0.214809 & & & 0.78 & 0 \\
\hline G4 & 64914.48 & -20 & 65004.83 & 65054.72 & 0.139192 & & 0.216046 & & & 0.84 & 0 \\
\hline
\end{tabular}




\section{LASR0238.CSV}

\begin{tabular}{|c|c|c|c|c|c|c|c|c|c|}
\hline$\sqrt{\mathrm{H1}}$ & 53735.73 & -20 & 53791.67 & 53824.9 & 0.104107 & 0.165948 & & 0.9 & 0 \\
\hline $\mathrm{H} 2$ & 62982.63 & -20 & 63058.5 & 63107.47 & 0.120463 & 0.198215 & & 0.96 & 0 \\
\hline $\mathrm{H3}$ & 71659.11 & -20 & 71801.91 & 71860.87 & 0.199278 & 0.281556 & & 1.02 & 0 \\
\hline $\mathrm{H} 4$ & 64586.12 & -20 & 64678.68 & 64726.64 & 0.14332 & 0.217568 & & 1.08 & 0 \\
\hline 11 & 49730.38 & -20 & 49787.59 & 49829.24 & 0.115044 & 0.198792 & 0.48271 & 1.14 & 0 \\
\hline 12 & 58625.88 & -20 & 58725.43 & 58755.79 & 0.169796 & 0.22159 & & & \\
\hline 13 & 76282.01 & -20 & 76385.3 & 76445.12 & 0.135405 & 0.213826 & & & \\
\hline 14 & 72378.99 & -20 & 72581.83 & 72663.83 & 0.280249 & 0.393536 & & & \\
\hline
\end{tabular}




\section{LASR0231.CSV}

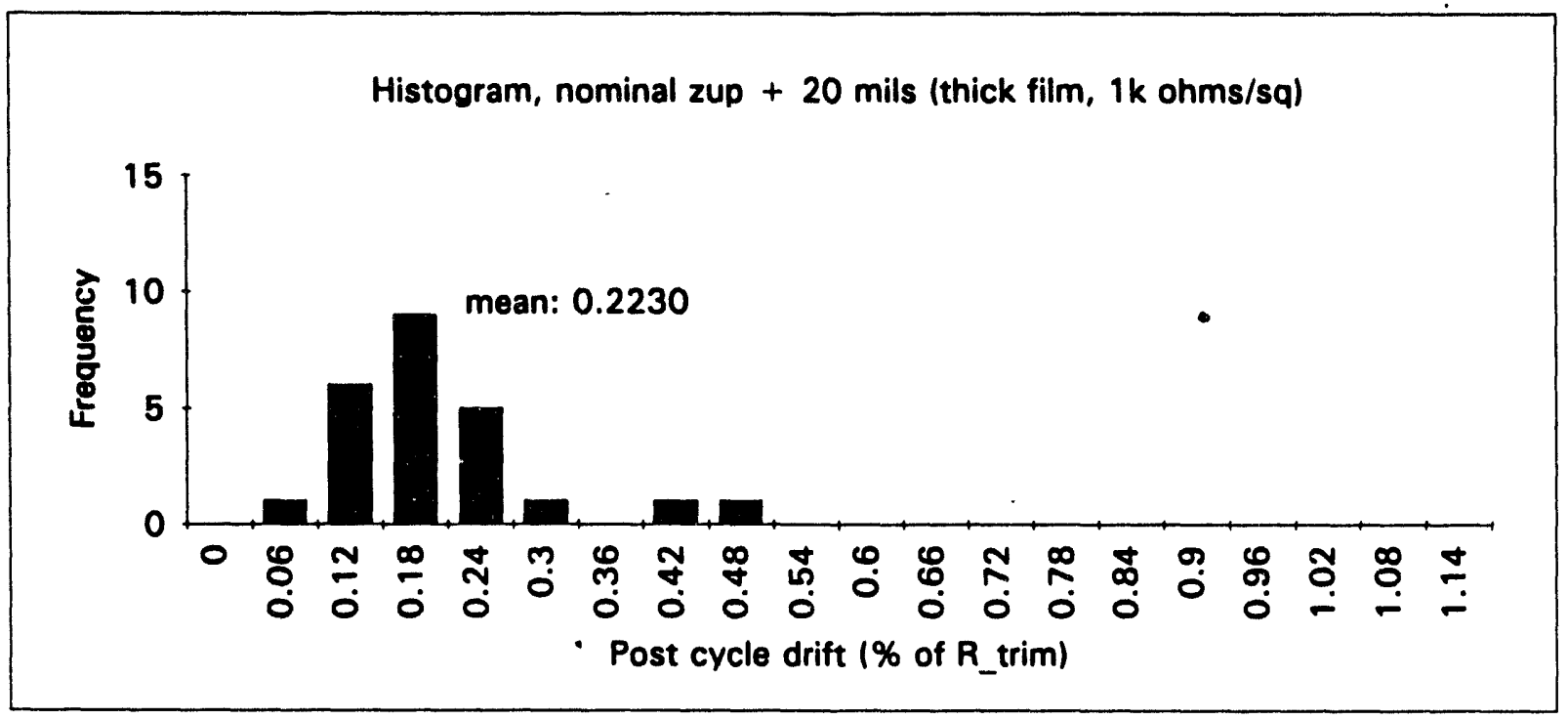

Histogram, nominal zup +0 mils (thick film, $1 k$ ohms/sq)

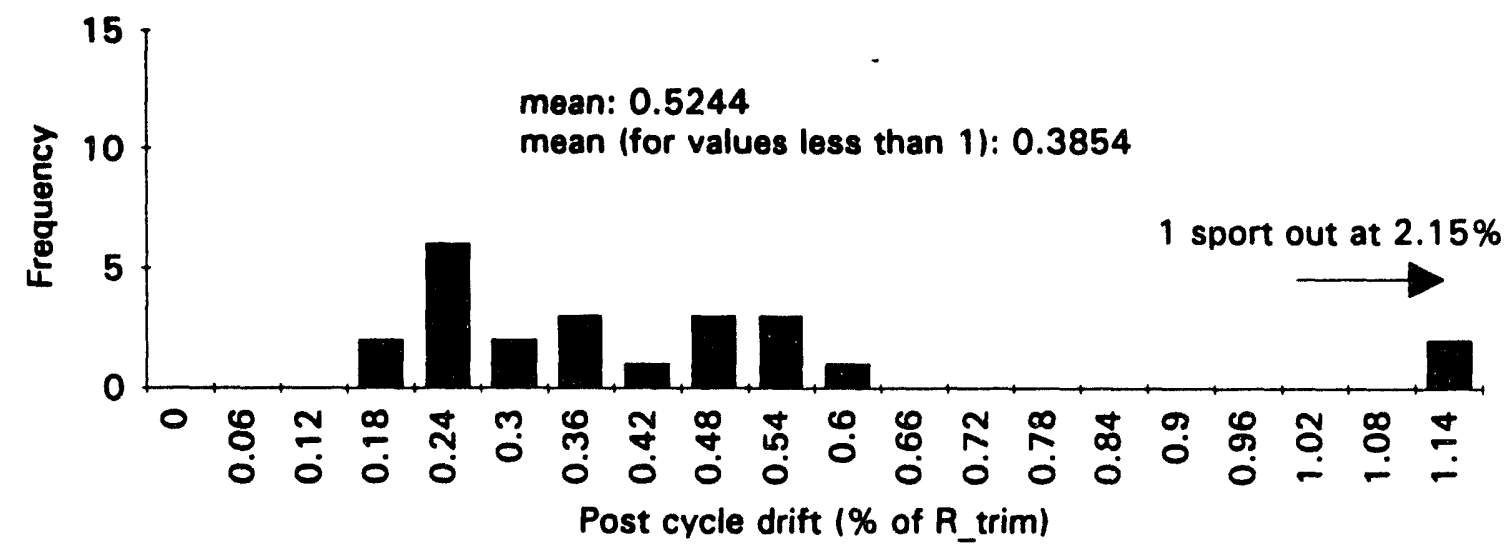

Histogram, nominal zup - 20 mils (thick film, 1k ohms/sq)

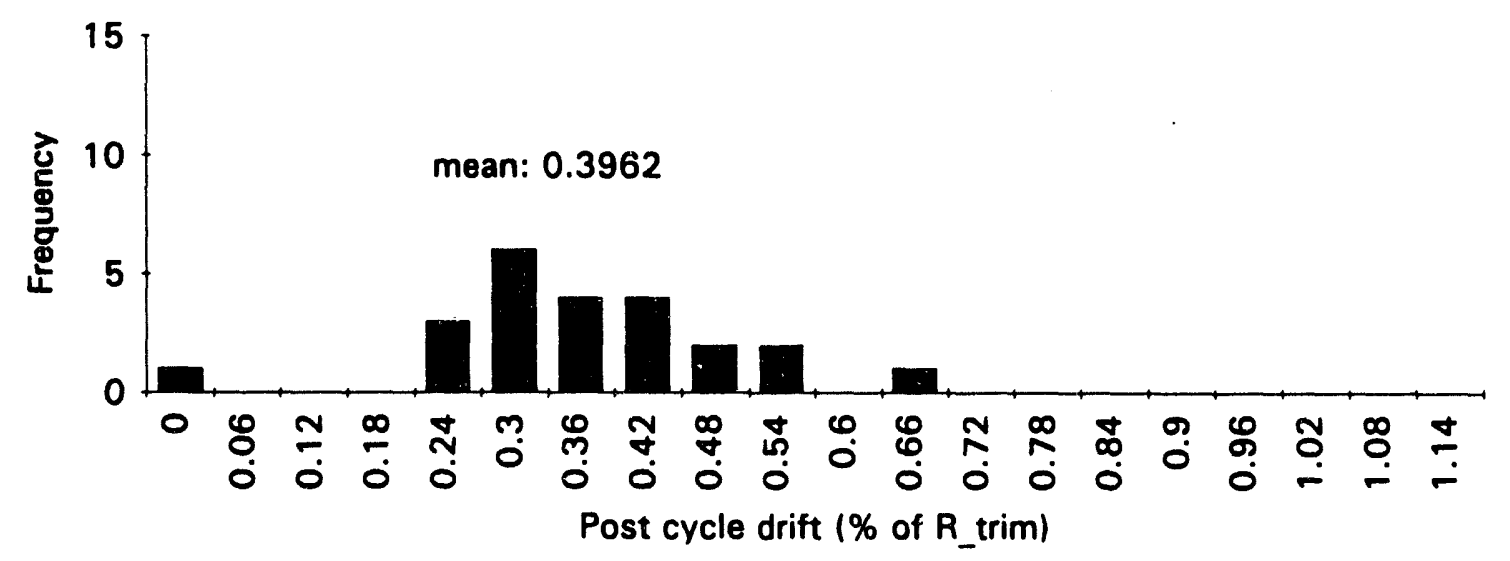




\begin{tabular}{|c|c|c|c|c|c|c|c|c|c|c|c|}
\hline HEI & RMAN TYPE: & THI & ICK FILM & & & & & & & & \\
\hline HEI & MAN S/N: & 306 & 8307 & & & & & & & & \\
\hline RES & ISTANCE P & ASTE: 1 & 000 & & & & & & & & \\
\hline DA & TE: $12 / 07 / 9$ & & & & & & & & & & \\
\hline TIN & IE: $16: 32: 0$ & & & & & & & & & & \\
\hline EXI & 'ERIMENT \# & $: 2$ & & & & & & & & & \\
\hline $\mathbf{R}$ & R_trim & z_ofst & R_24 & R_post & R_24_drift & R_24_avg & R_post_drif & R_post_avg & bin_range & $\operatorname{Bin}$ & Frequency \\
\hline A1 & 6241.061 & 20 & 6246.599 & 6256.58 & 0.088734 & 0.089793 & 0.248661 & \begin{tabular}{|l|}
0.22303 \\
\end{tabular} & \begin{tabular}{|l|}
0 \\
\end{tabular} & 0 & 0 \\
\hline A2 & 5069.25 & 20 & 5079.605 & 5094.049 & 0.204269 & & 0.489196 & & 0.06 & 0.06 & 1 \\
\hline A3 & 4900.588 & 20 & 4904.413 & 4909.742 & 0.078046 & & 0.1868 & & 0.12 & 0.12 & 6 \\
\hline A4 & 6078.236 & 20 & 6082.46 & 6086.607 & 0.069493 & & 0.137723 & & 0.18 & 0.18 & 9 \\
\hline B1 & 5837.659 & 20 & 5843.103 & 5850.799 & 0.093262 & & 0.225095 & & 0.24 & 0.24 & 5 \\
\hline$B 2$ & 4645.33 & 20 & 4648.244 & 4654.151 & 0.062731 & & 0.189894 & & 0.3 & 0.3 & 1 \\
\hline B3 & 4773.604 & 20 & 4776.418 & 4783.995 & 0.058951 & & 0.217669 & & 0.36 & 0.36 & 0 \\
\hline B4 & 6545.919 & 20 & 6554.758 & 6561.757 & 0.135031 & & 0.241959 & & 0.42 & 0.42 & 1 \\
\hline C1 & 5523.293 & 20 & 5526.509 & 5532.256 & 0.058221 & & 0.162271 & & 0.48 & 0.48 & 1 \\
\hline $\mathrm{C} 2$ & 4145.564 & 20 & 4147.381 & 4152.042 & 0.043819 & & 0.156267 & & 0.54 & 0.54 & 0 \\
\hline C3 & 4964.992 & 20 & 4968.436 & 4975.936 & 0.069369 & & 0.220432 & & 0.6 & 0.6 & 0 \\
\hline C4 & 7836.492 & 20 & 7844.014 & 7852.825 & 0.095988 & & 0.208425 & & 0.66 & 0.66 & 0 \\
\hline A1 & 5888.003 & 20 & 5897.285 & 5914.692 & 0.157646 & & 0.453274 & & 0.72 & 0.72 & 0 \\
\hline$\overline{A 2}$ & 5193.179 & 20 & 5199.114 & 5209.687 & 0.114281 & & 0.317876 & & 0.78 & 0.78 & 0 \\
\hline A3 & 5248.171 & 20 & 5252.513 & 5262.12 & 0.082727 & & 0.265781 & & 0.84 & 0.84 & 0 \\
\hline A4 & 5628.092 & 20 & 5632.068 & 5638.446 & 0.070642 & & 0.183962 & & 0.9 & 0.9 & 0 \\
\hline B1 & 5327.044 & 20 & 5331.204 & 5336.166 & 0.078097 & & 0.171247 & & 0.96 & 0.96 & 0 \\
\hline B2 & 5136.715 & 20 & 5141.233 & 5150.825 & 0.087948 & & 0.274697 & & 1.02 & 1.02 & 0 \\
\hline B3 & 5164.901 & 20 & 5170.571 & 5177.542 & 0.109781 & & 0.244741 & & 1.08 & 1.08 & 0 \\
\hline B4 & 6098.186 & 20 & 6101.685 & 6106.059 & 0.057374 & & 0.129097 & & 1.14 & 1.14 & 0 \\
\hline C1 & 5566.889 & 20 & 5570.872 & 5576.974 & 0.071556 & & 0.181163 & & & & \\
\hline C2 & 4579.027 & 20 & 4581.361 & 4583.012 & 0.050964 & & 0.087017 & & & & \\
\hline C3 & 5327.053 & 20 & 5332.476 & 5338.261 & 0.101793 & & 0.210391 & & & & \\
\hline C4 & 6980.315 & 20 & 6988.293 & 6990.721 & 0.114298 & & 0.14907 & & & & \\
\hline D1 & 4278.598 & 0 & 4282.042 & 4294.451 & 0.080487 & 0.140496 & 0.370515 & 0.524357 & & Bin & Frequency \\
\hline $\mathrm{D} 2$ & 4170.427 & 0 & 4174.924 & 4186.081 & 0.107832 & & 0.375356 & 0.385379 & & 0 & 0 \\
\hline D3 & 4887.696 & 0 & 4895.835 & 4917.87 & 0.166514 & & 0.617352 & & & 0.06 & 0 \\
\hline D4 & -5810.071 & 0 & 5817.323 & 5823.877 & 0.124824 & & 0.237617 & & & 0.12 & 0 \\
\hline
\end{tabular}




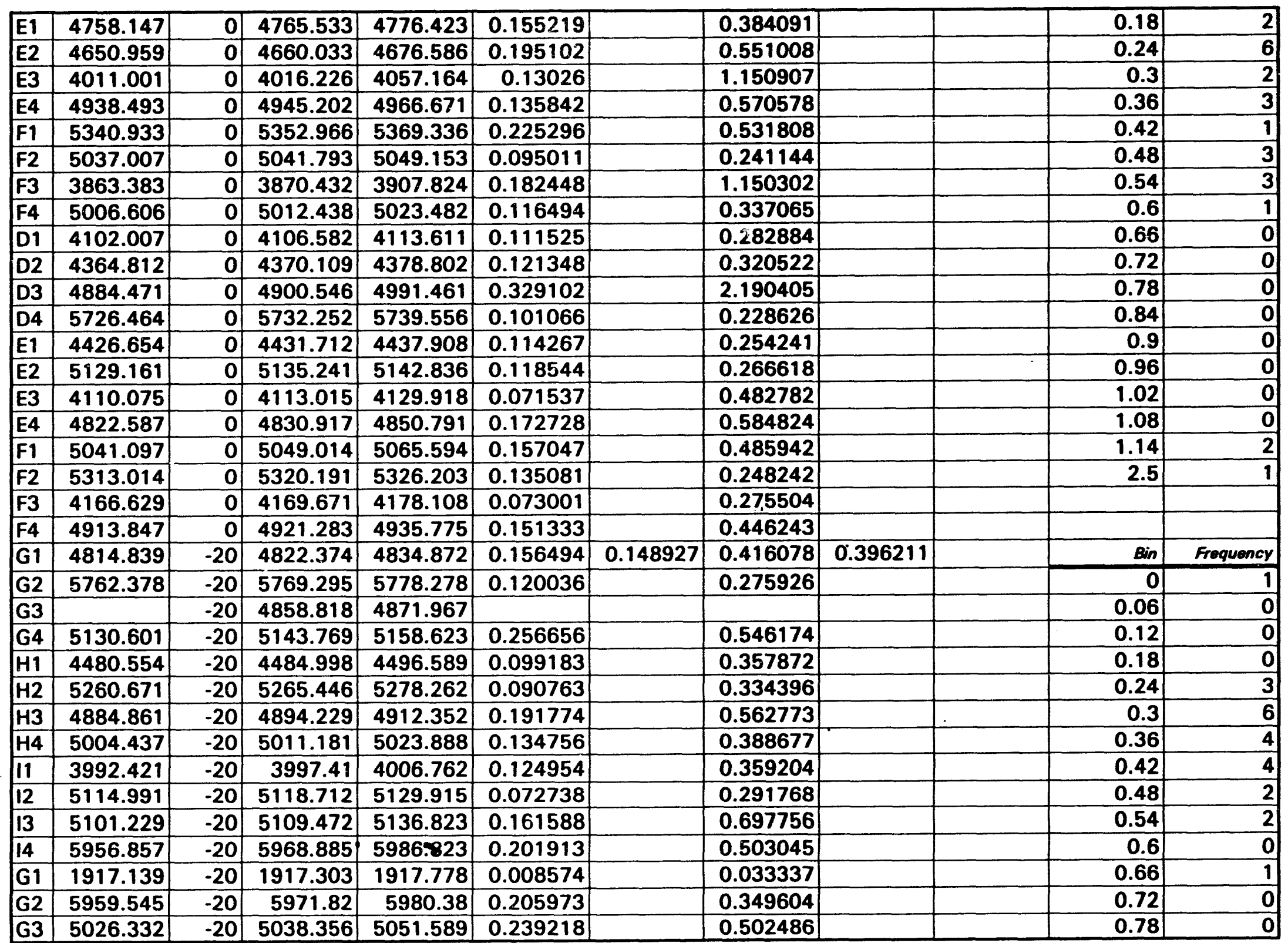


LASR0231.CSV

\begin{tabular}{|c|c|c|c|c|c|c|c|c|}
\hline G4 & 5331.687 & -20 & 5338.719 & 5345.206 & 0.131894 & 0.253562 & 0.84 & 0 \\
\hline $\mathrm{H1}$ & 4386.694 & -20 & 4394.64 & 4403.942 & 0.181142 & 0.393194 & 0.9 & 0 \\
\hline $\mathrm{H} 2$ & 5517.153 & -20 & 5526.053 & 5536.046 & 0.16132 & 0.342439 & 0.96 & 0 \\
\hline $\mathrm{H3}$ & 5352.471 & -20 & 5360.348 & 5373.646 & 0.147168 & 0.395604 & 1.02 & 0 \\
\hline $\mathrm{H} 4$ & 5080.443 & -20 & 5090.756 & 5104.758 & 0.202987 & 0.478599 & 1.08 & 0 \\
\hline 11 & 4016.457 & -20 & 4022.849 & 4034.413 & 0.159137 & 0.447071 & 1.14 & 0 \\
\hline 12 & 4905.663 & -20 & 4910.428 & 4921.188 & 0.097129 & 0.316468 & & \\
\hline 13 & 5329.585 & -20 & 5336.662 & 5353.316 & 0.132778 & 0.445274 & & \\
\hline 14 & 5732.41 & -20 & 5740.845 & 5756.575 & 0.147143 & 0.421544 & & \\
\hline
\end{tabular}




\section{LASR0220.CSV}

Histogram, nominal zup +20 mils (thick film, $100 \mathrm{ohms} / \mathrm{sq}$ )

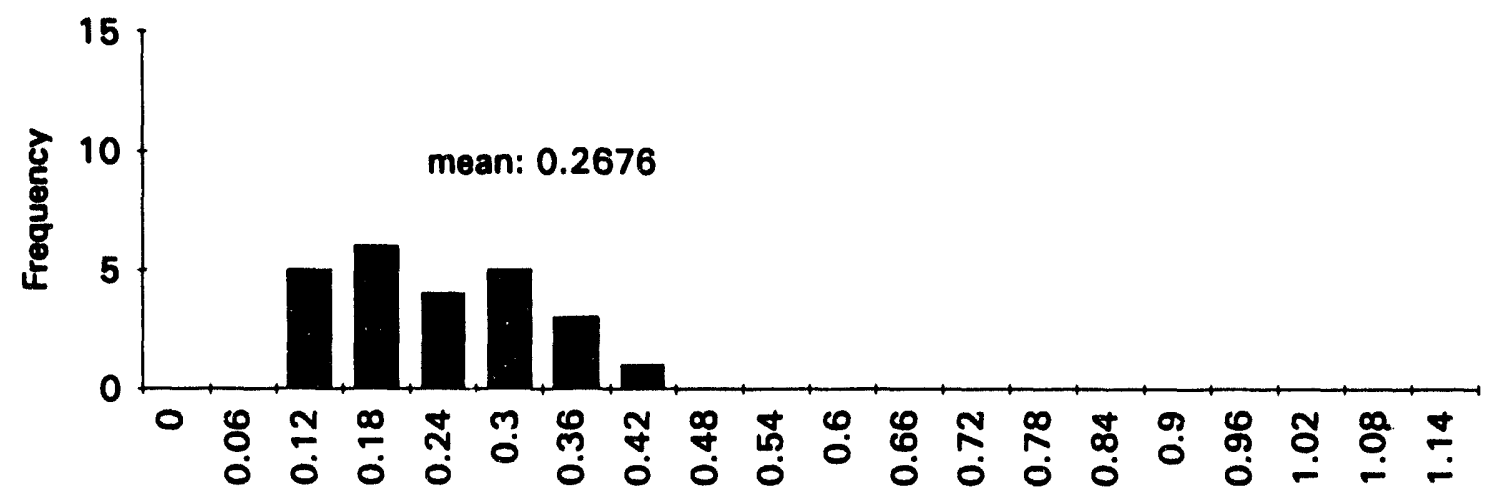

Post cycle drift (\% of R_trim)

Histogram, nominal zup +0 mils (thick film, $100 \mathrm{ohms} / \mathrm{sq}$ )

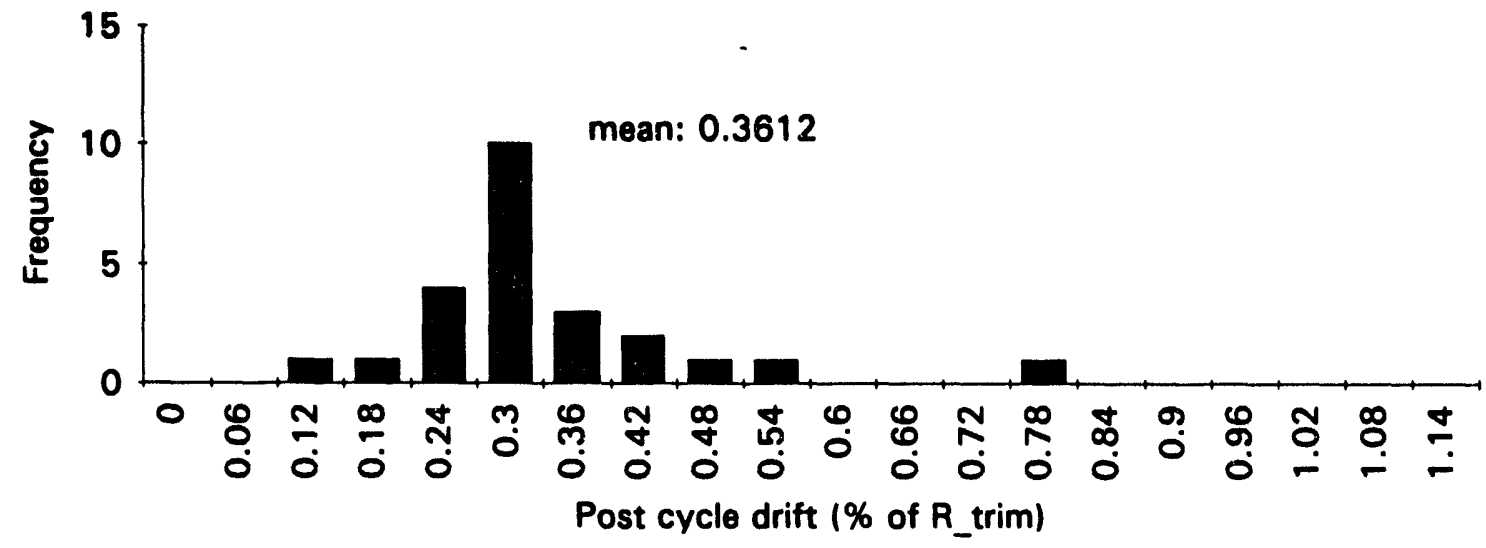

Histogram, nominal zup - 20 mils (thick film, 100 ohms/sq)

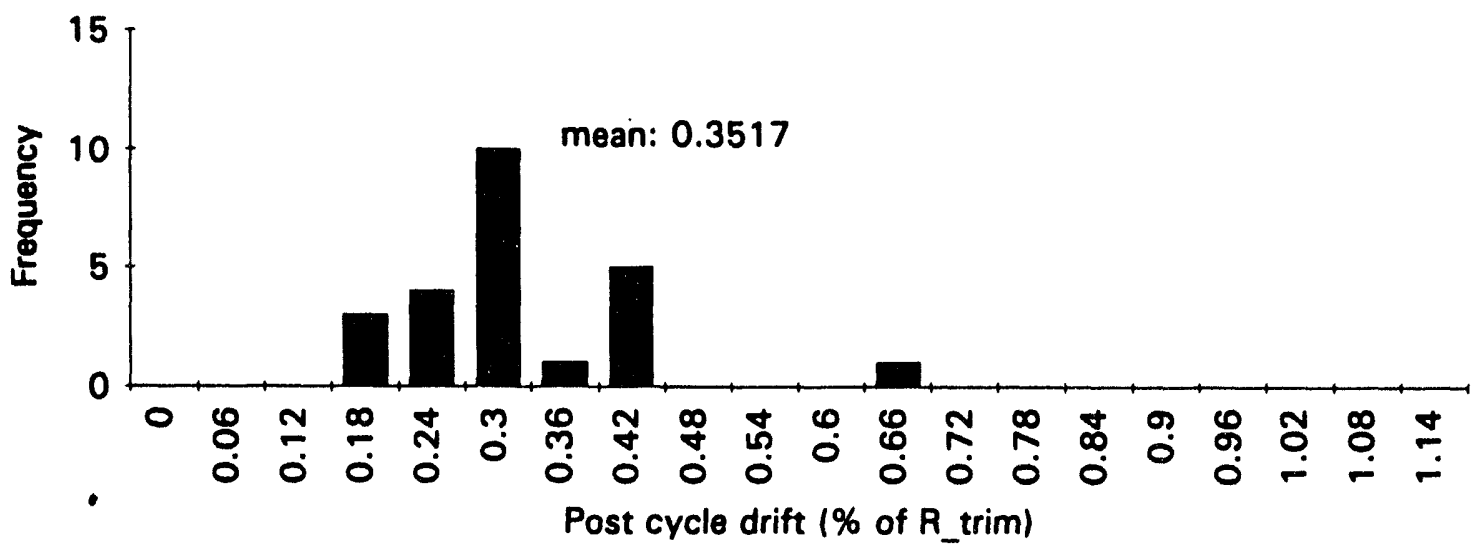




\section{LASR0220.CSV}

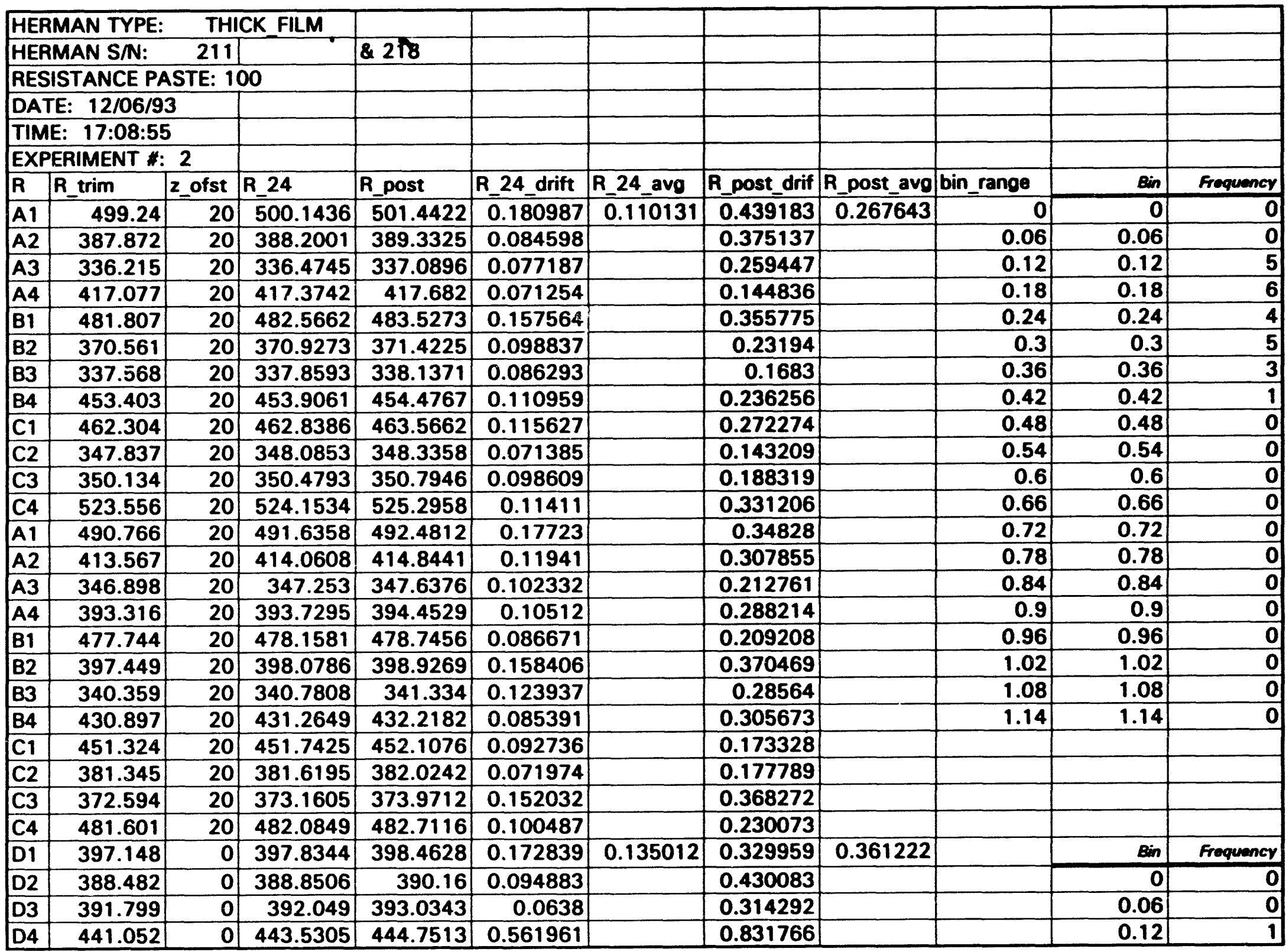




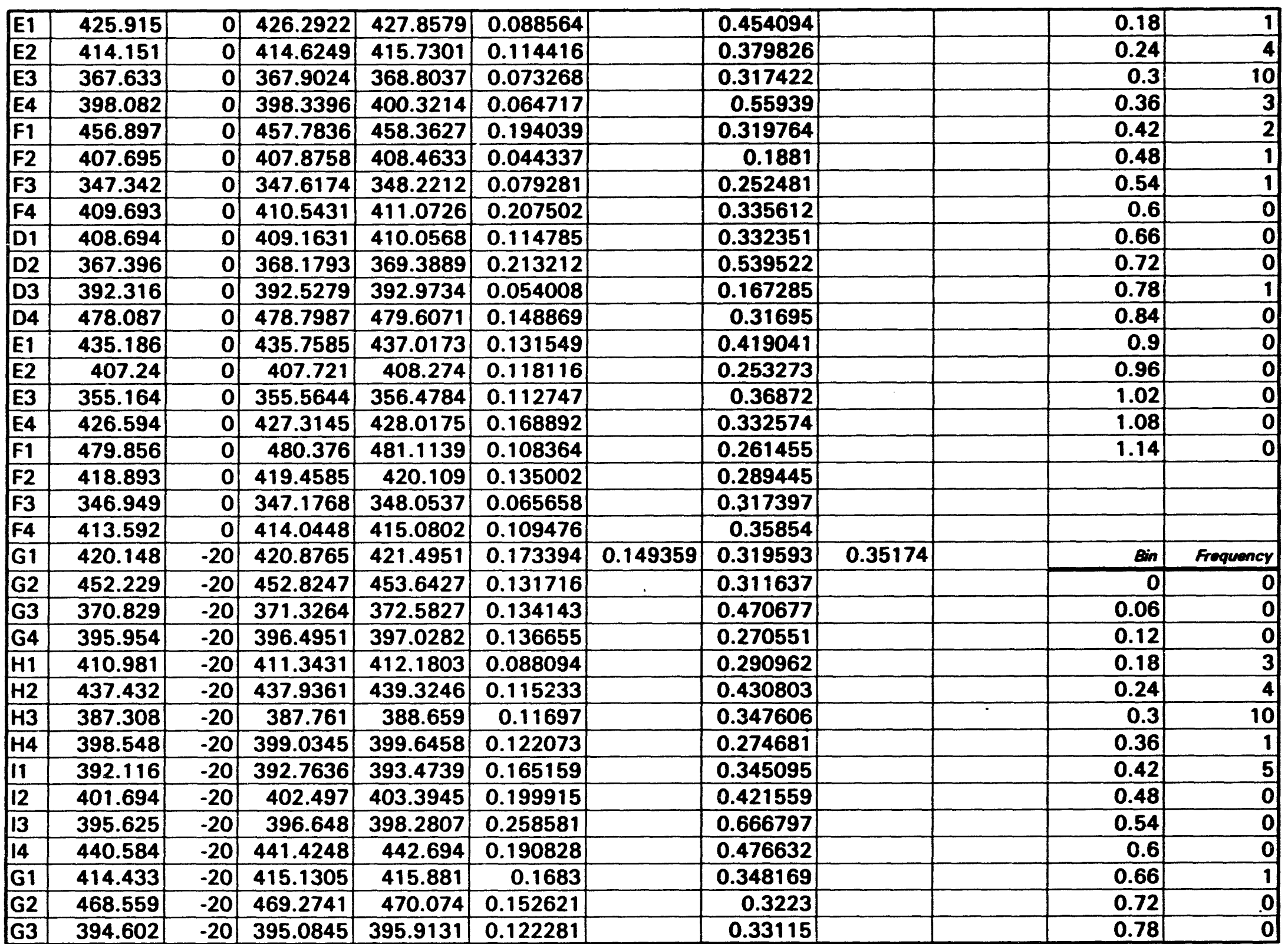


LASR0220.CSV

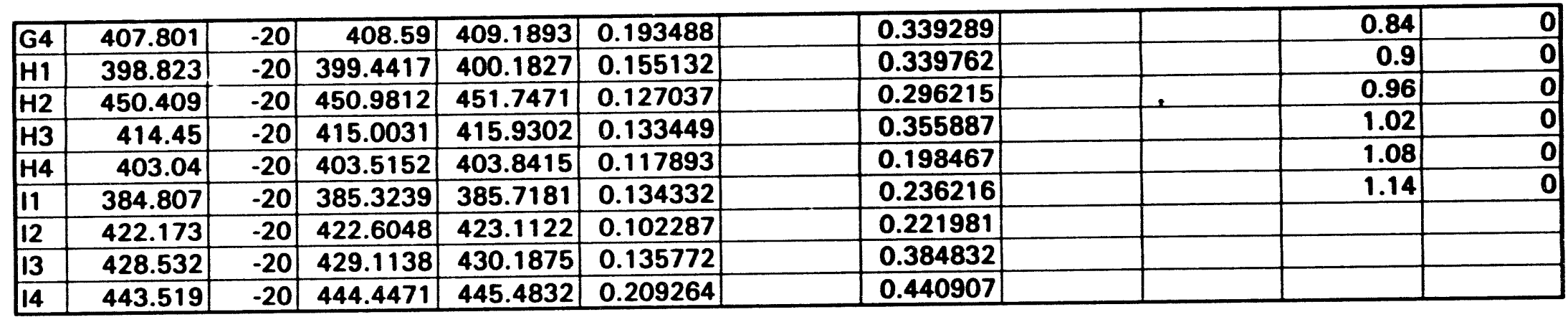




\section{LASR0218.CSV}
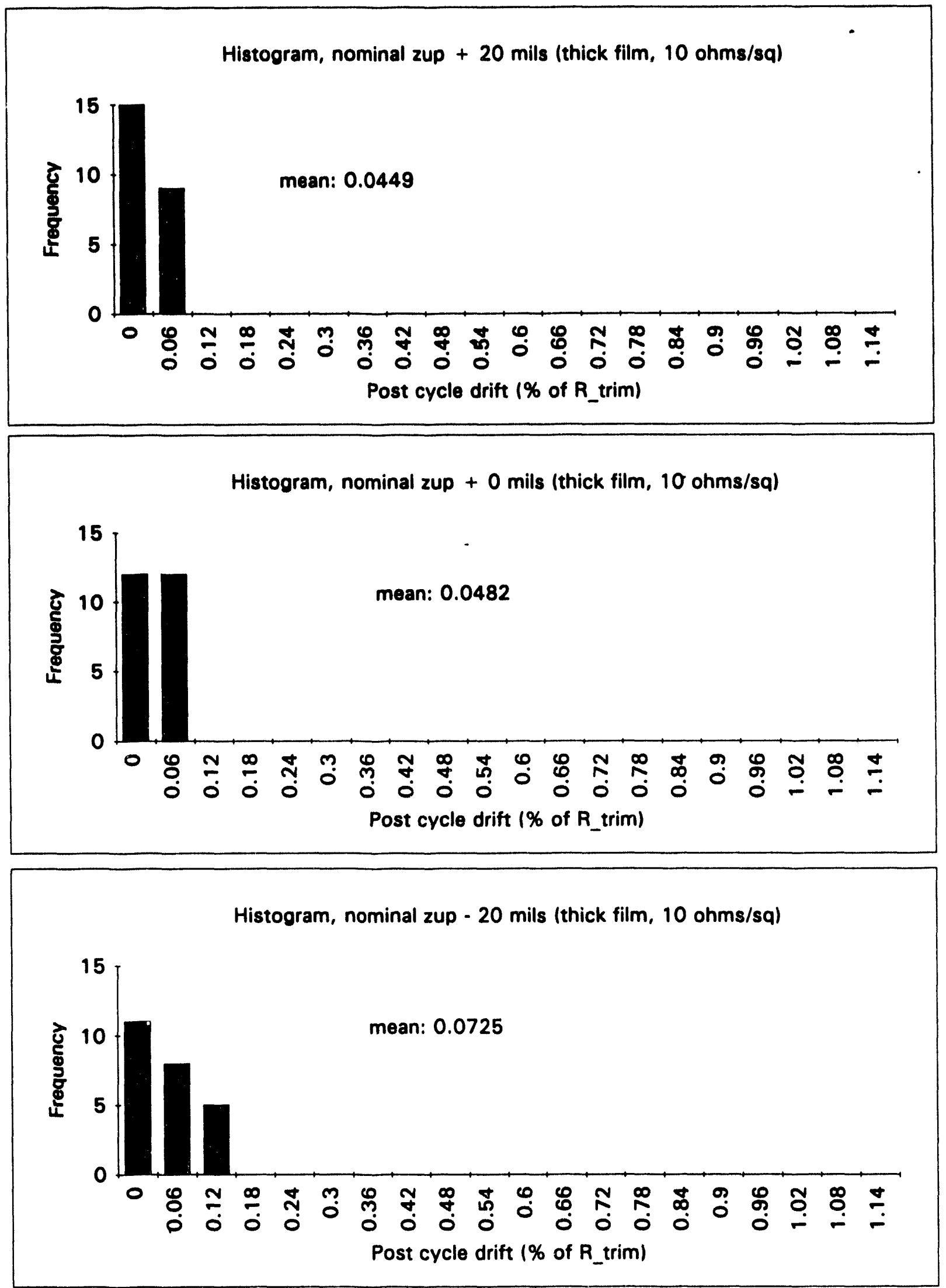


\section{LASR0218.CSV}
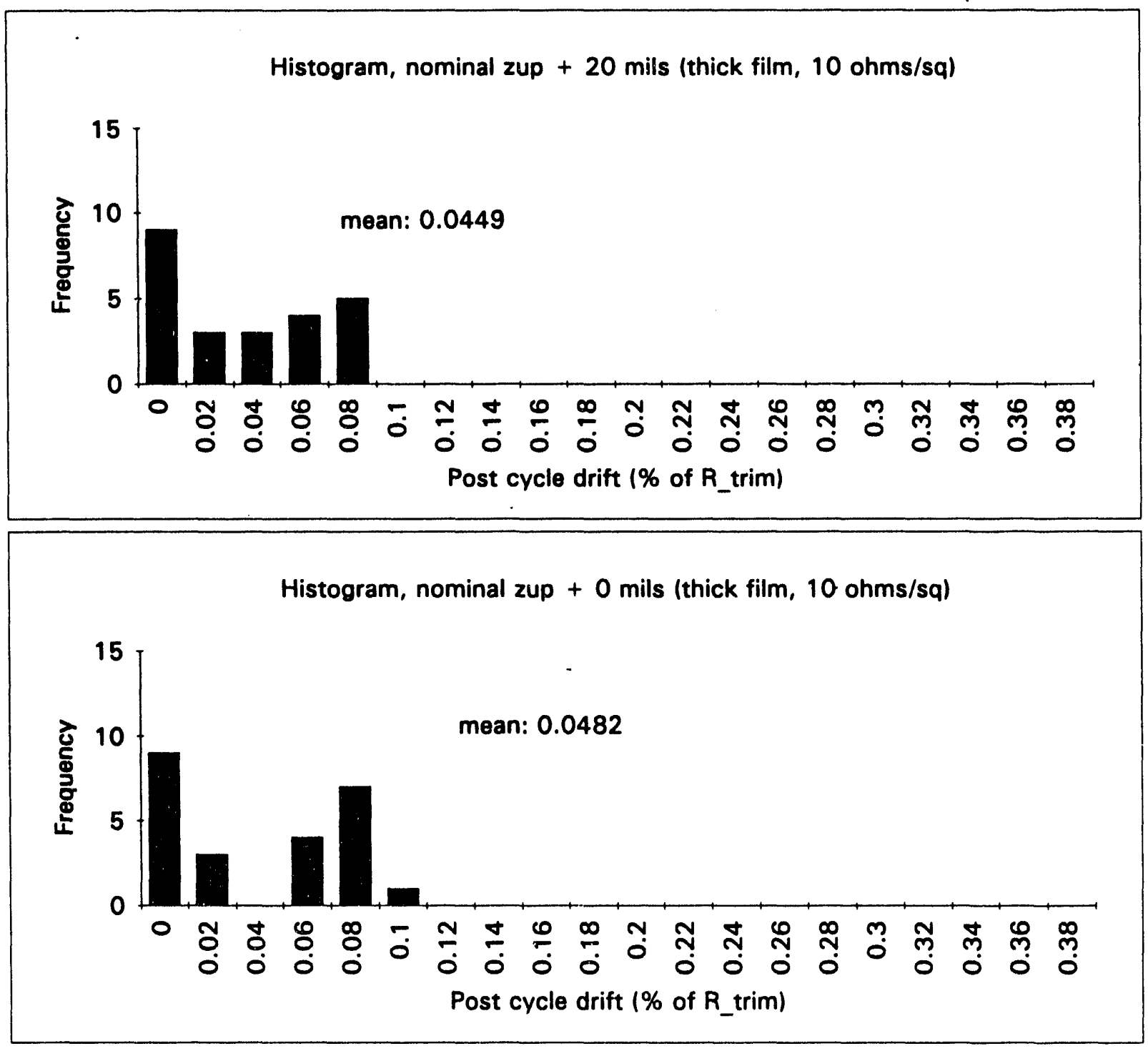

Histogram, nominal zup - 20 mils (thick film, 10 ohms/sq)

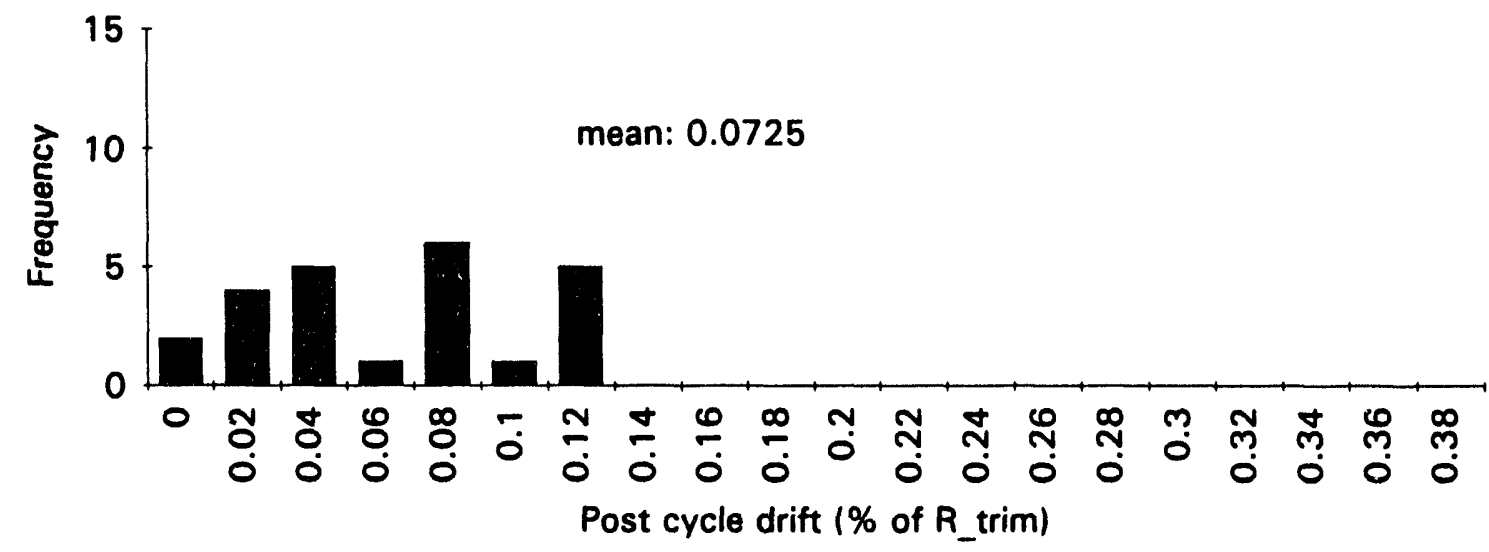




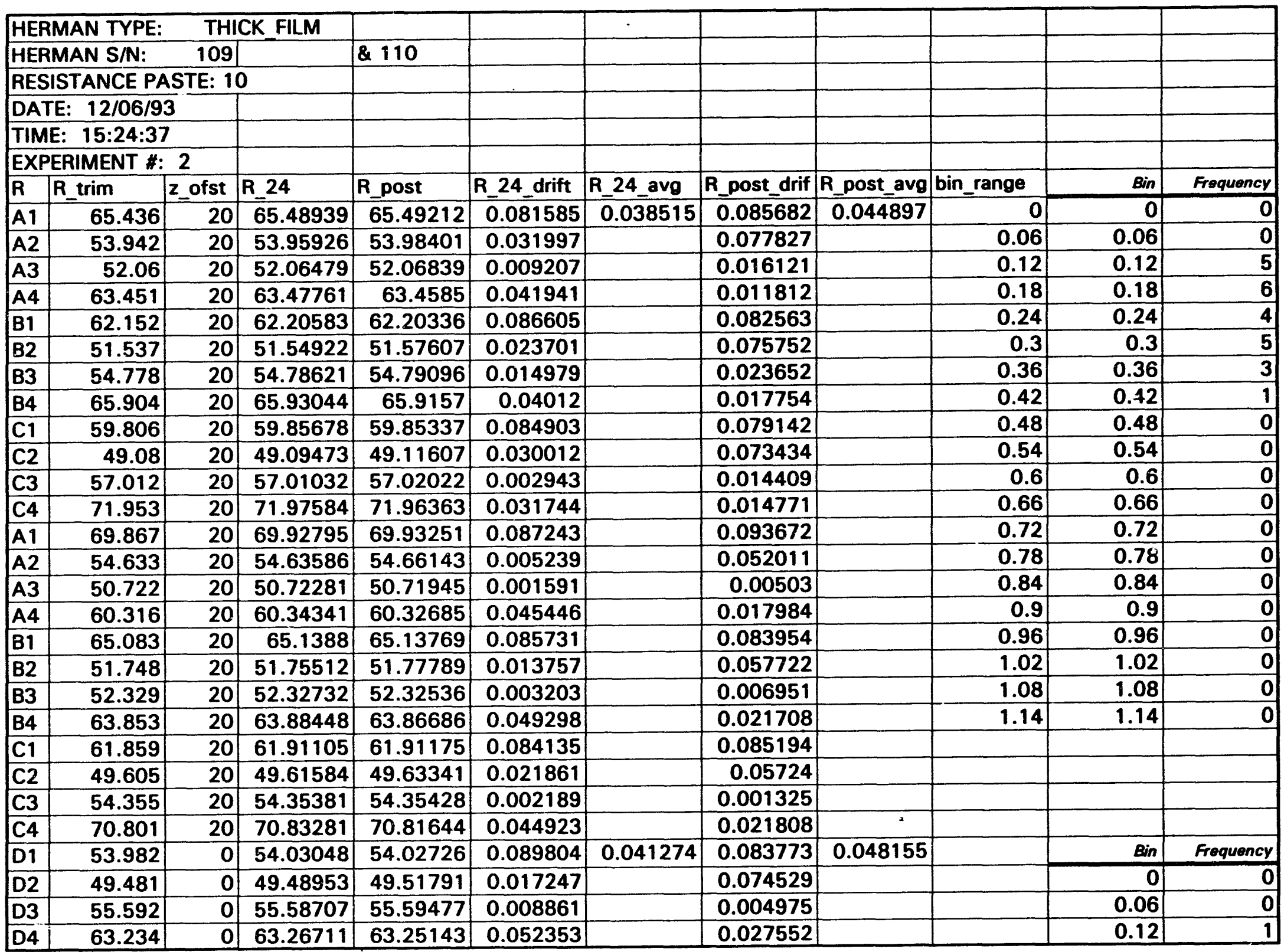




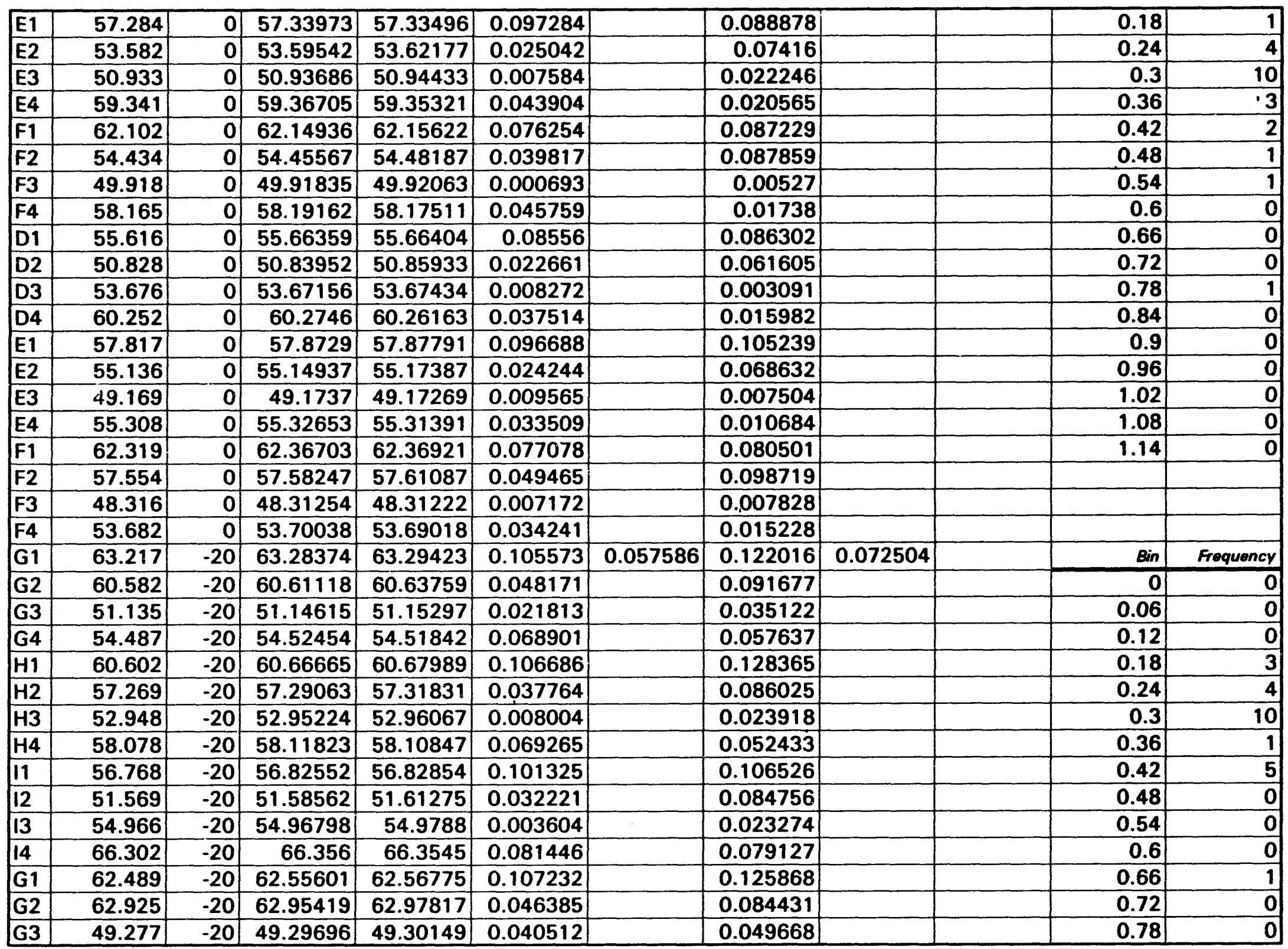


LASR0218.CSV

\begin{tabular}{|c|c|c|c|c|c|c|c|c|}
\hline$\longdiv { \text { G4 } }$ & 51.508 & -20 & 51.53879 & 51.52964 & 0.059771 & 0.041989 & 0.84 & 0 \\
\hline H1 & 60.932 & -20 & 61.00324 & 61.01265 & 0.116919 & 0.132191 & 0.9 & 0 \\
\hline $\mathrm{H} 2$ & 58.514 & -20 & 58.54143 & 58.56456 & 0.046883 & 0.086334 & 0.96 & 0 \\
\hline H3 & 51.322 & -20 & 51.32373 & 51.32877 & 0.003375 & 0.01318 & 1.02 & 0 \\
\hline $\mathrm{H} 4$ & 53.187 & -20 & 53.21151 & 53.20316 & 0.046088 & 0.030365 & 1.08 & 0 \\
\hline 11 & 57.497 & -20 & 57.56314 & 57.57149 & 0.11503 & 0.129382 & 1.14 & 0 \\
\hline 12 & 53.377 & -20 & 53.39667 & 53.42225 & 0.036853 & 0.084706 & & \\
\hline 13 & 53.205 & -20 & 53.21126 & 53.21458 & 0.011762 & 0.017995 & & \\
\hline 14 & 61.836 & -20 & 61.87711 & 61.86887 & 0.066484 & 0.053122 & & \\
\hline
\end{tabular}


Appendix F

\section{Thin Film Characterization Data}


24 Hour Resistence Drift (Q-rate $=2 \mathrm{kHz}$, Thin Film)

Resistance Drift (\% of R_trim)

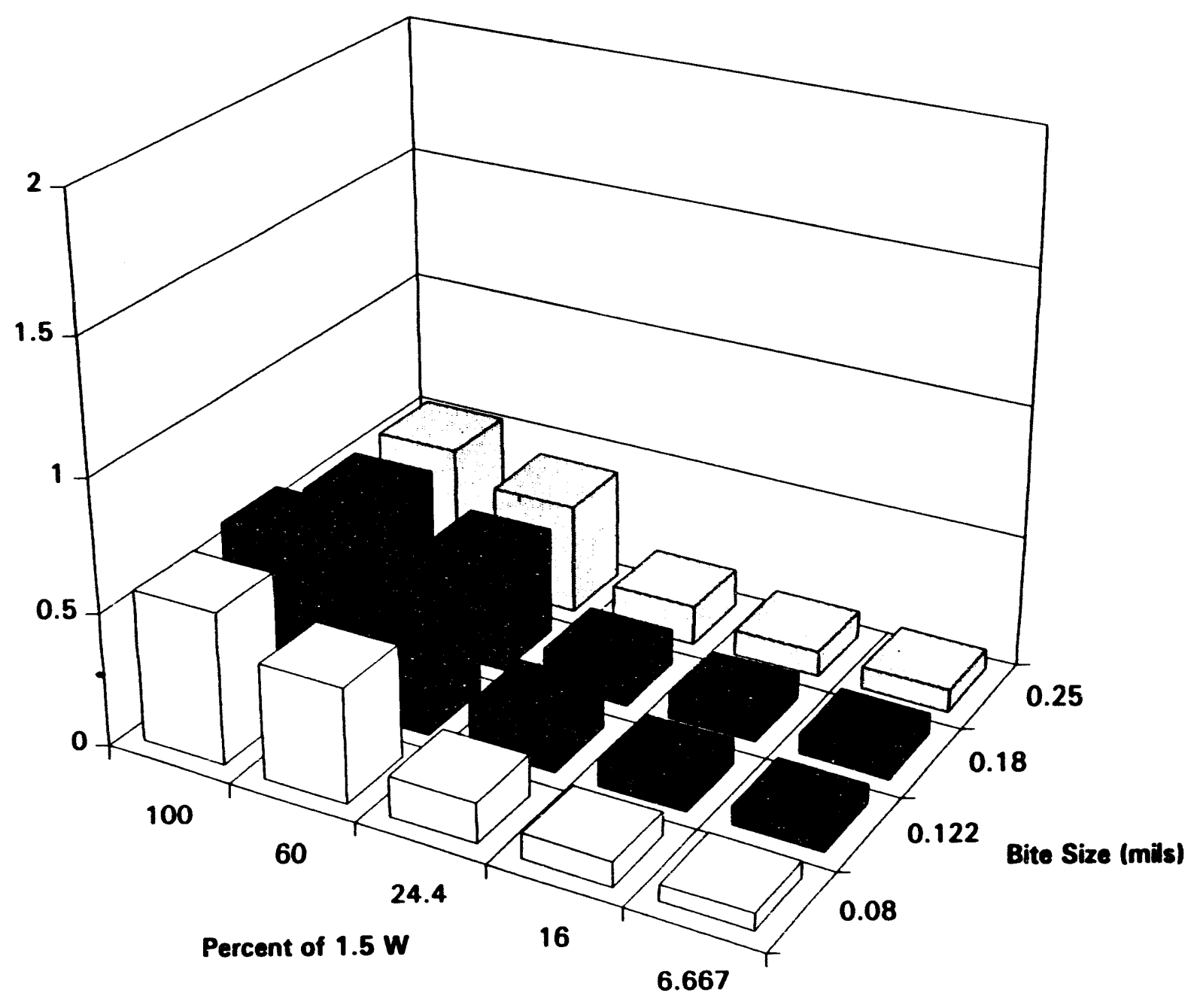


24 Hour Resistence Drift (Q-rate $=3 \mathrm{kHz}$, Thin Film)

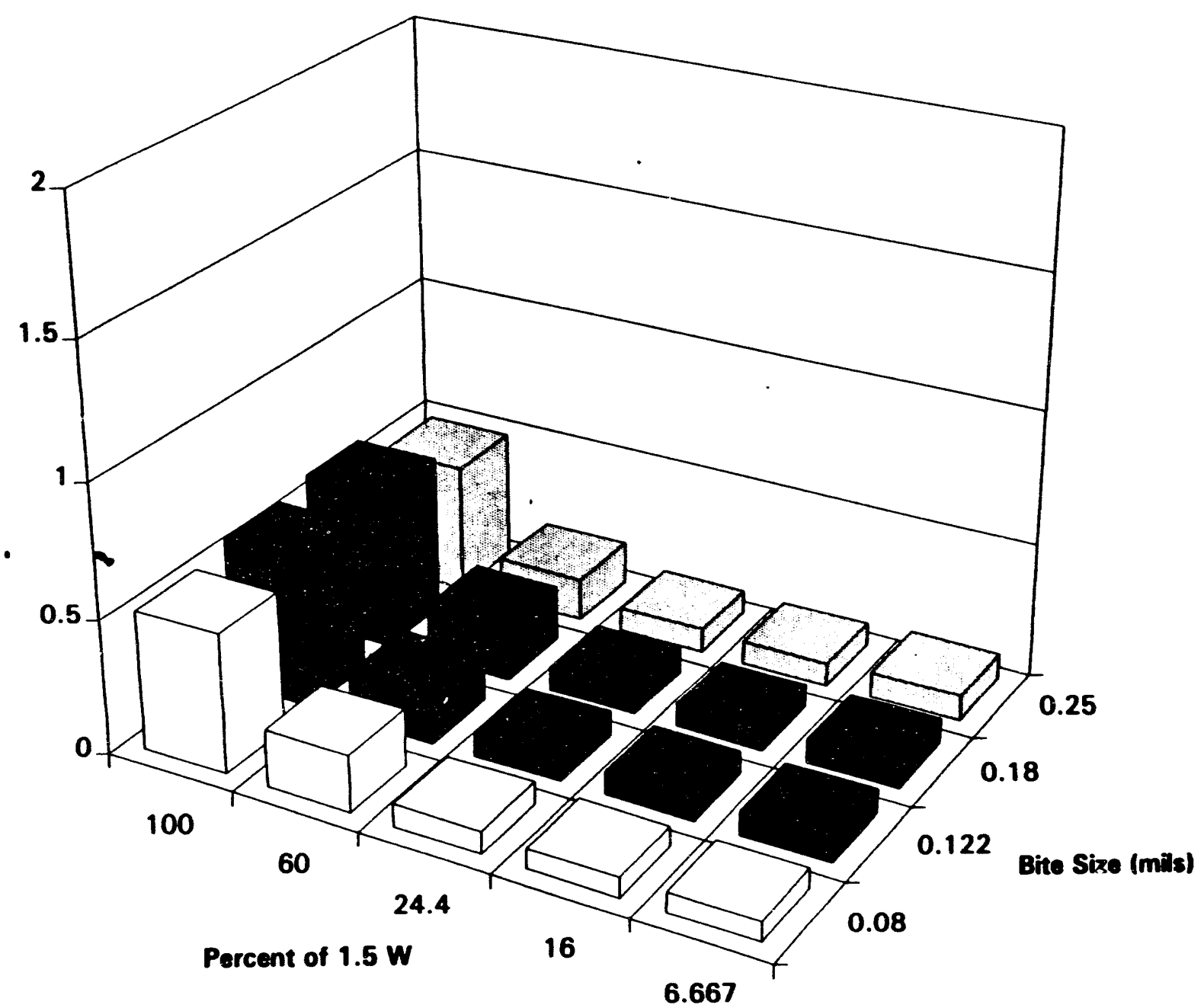


24 Hour Resistance Drift (Q-rate $=4 \mathrm{kHz}$, Thin Film)

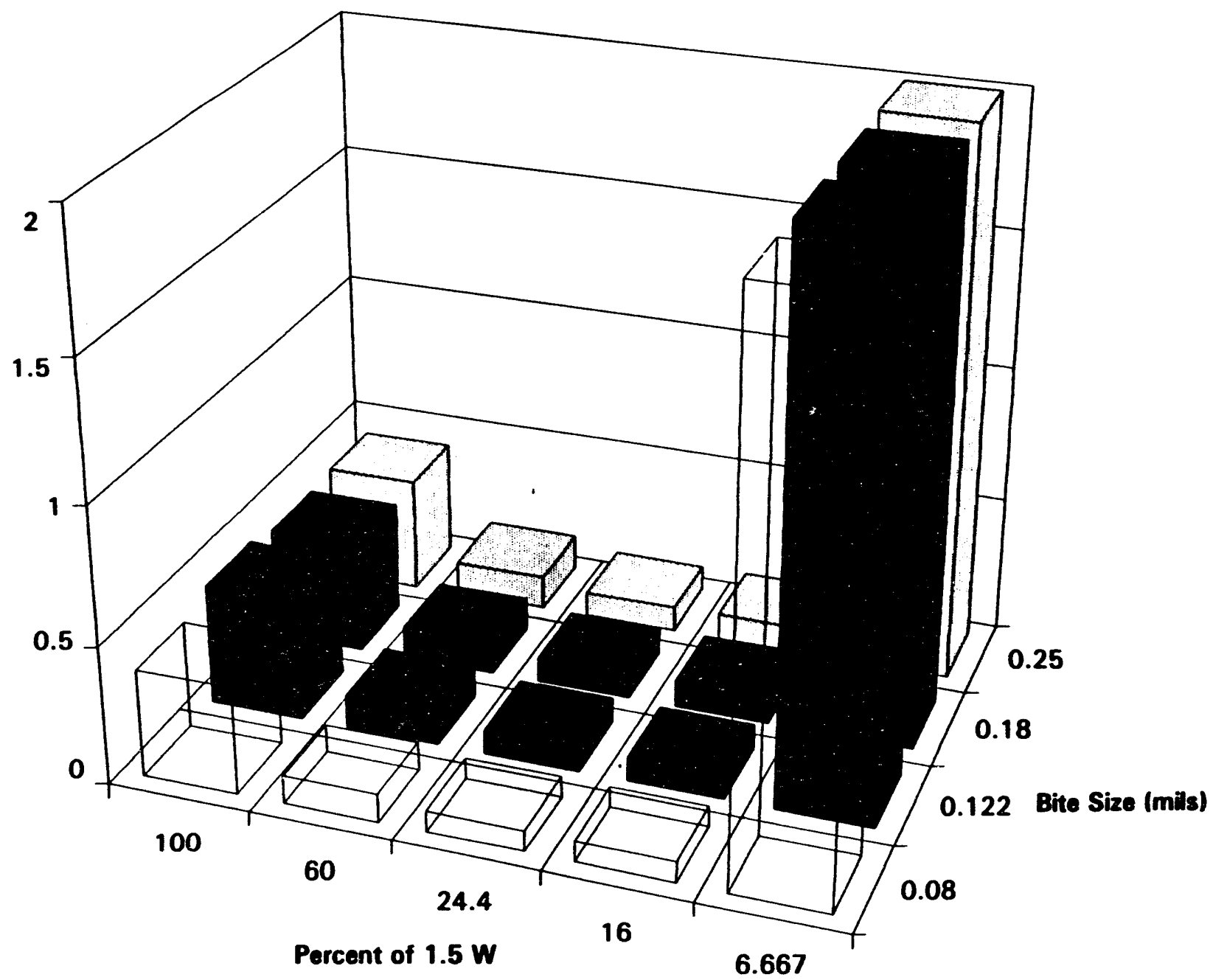




\section{Post Life Test Resistance Drift (Q-rate $=2 \mathrm{kHz}$, Thin Film)}

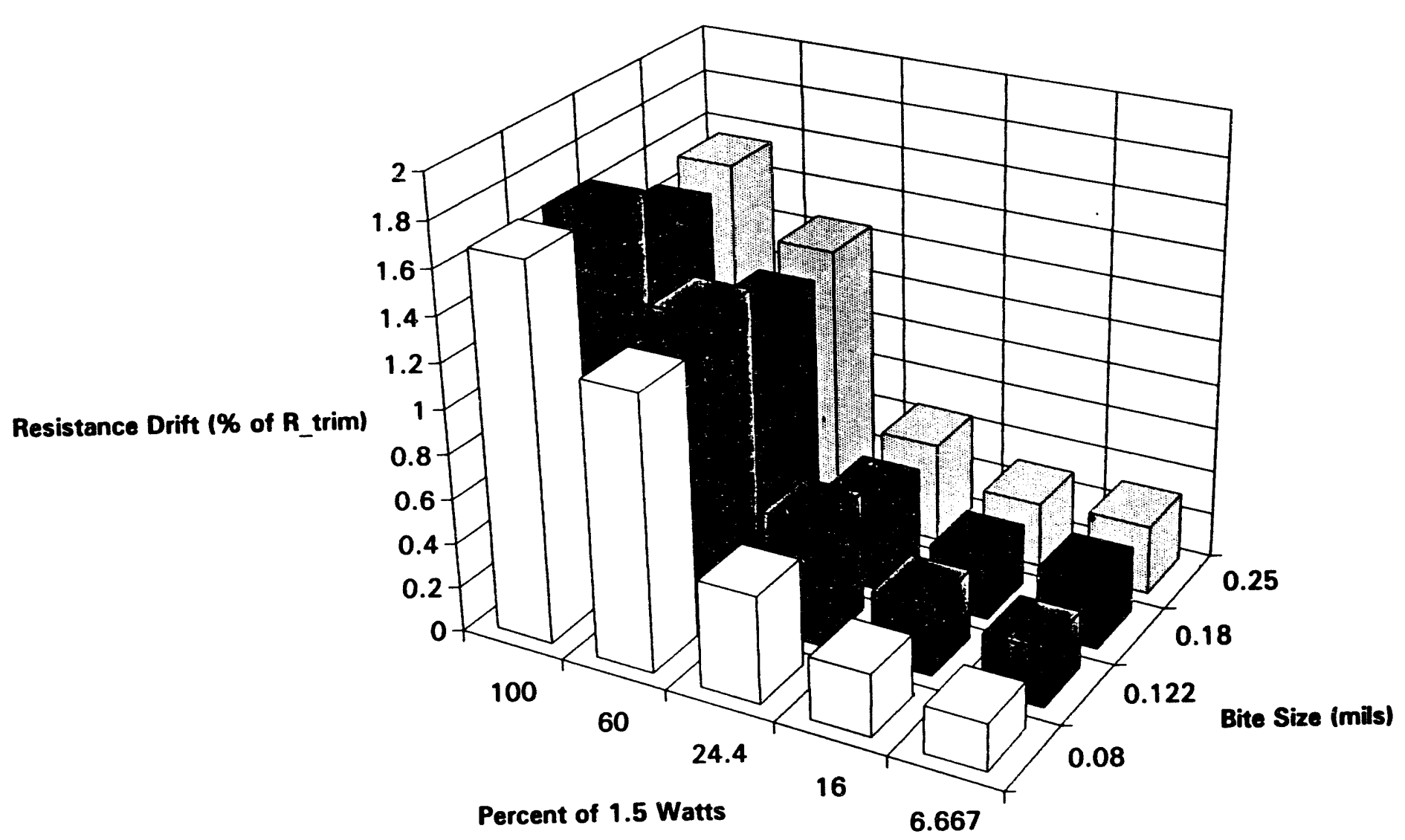




\section{Post Life Test Resistance Drift (Q-rate $=3 \mathrm{kHz}$, Thin Film)}

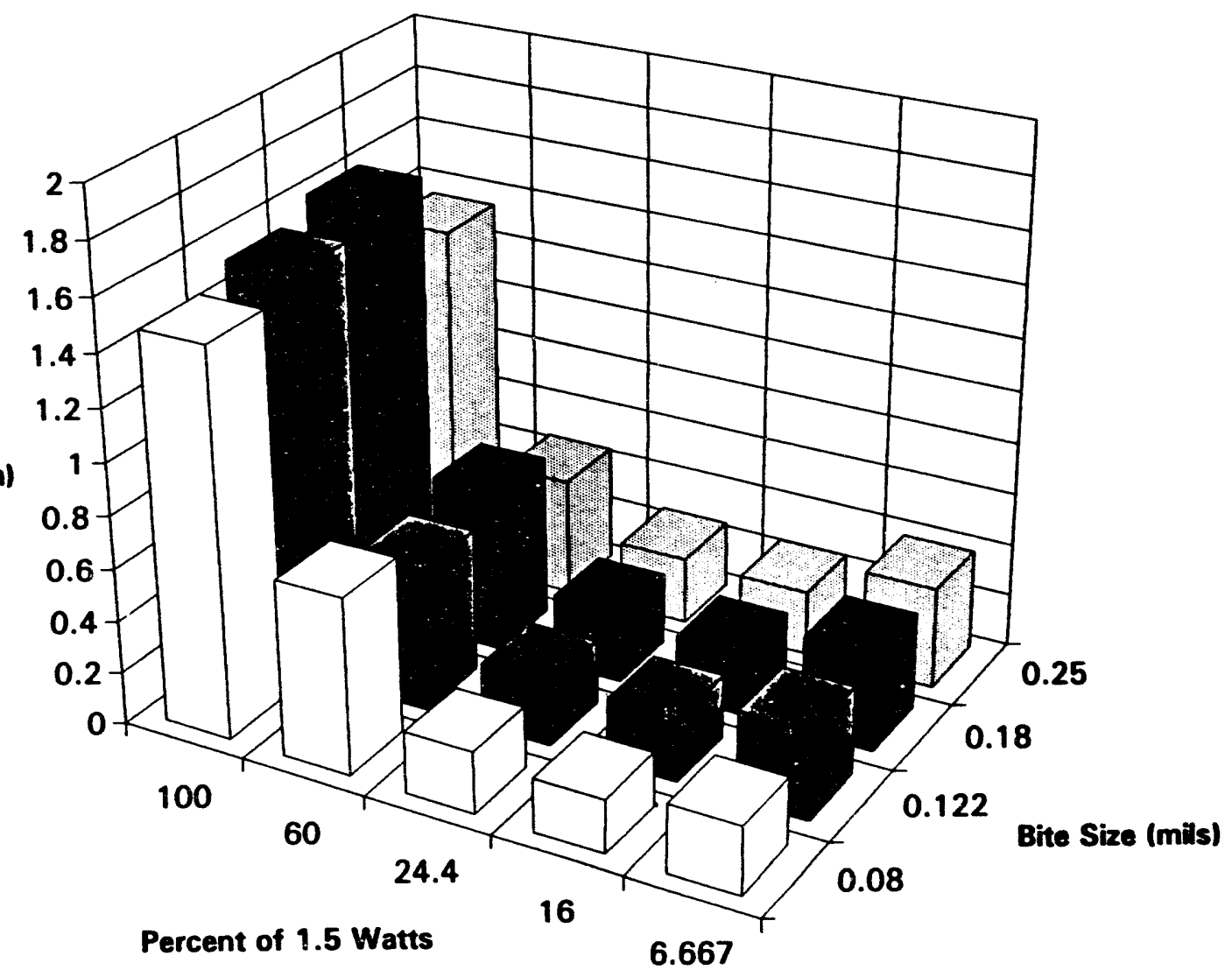




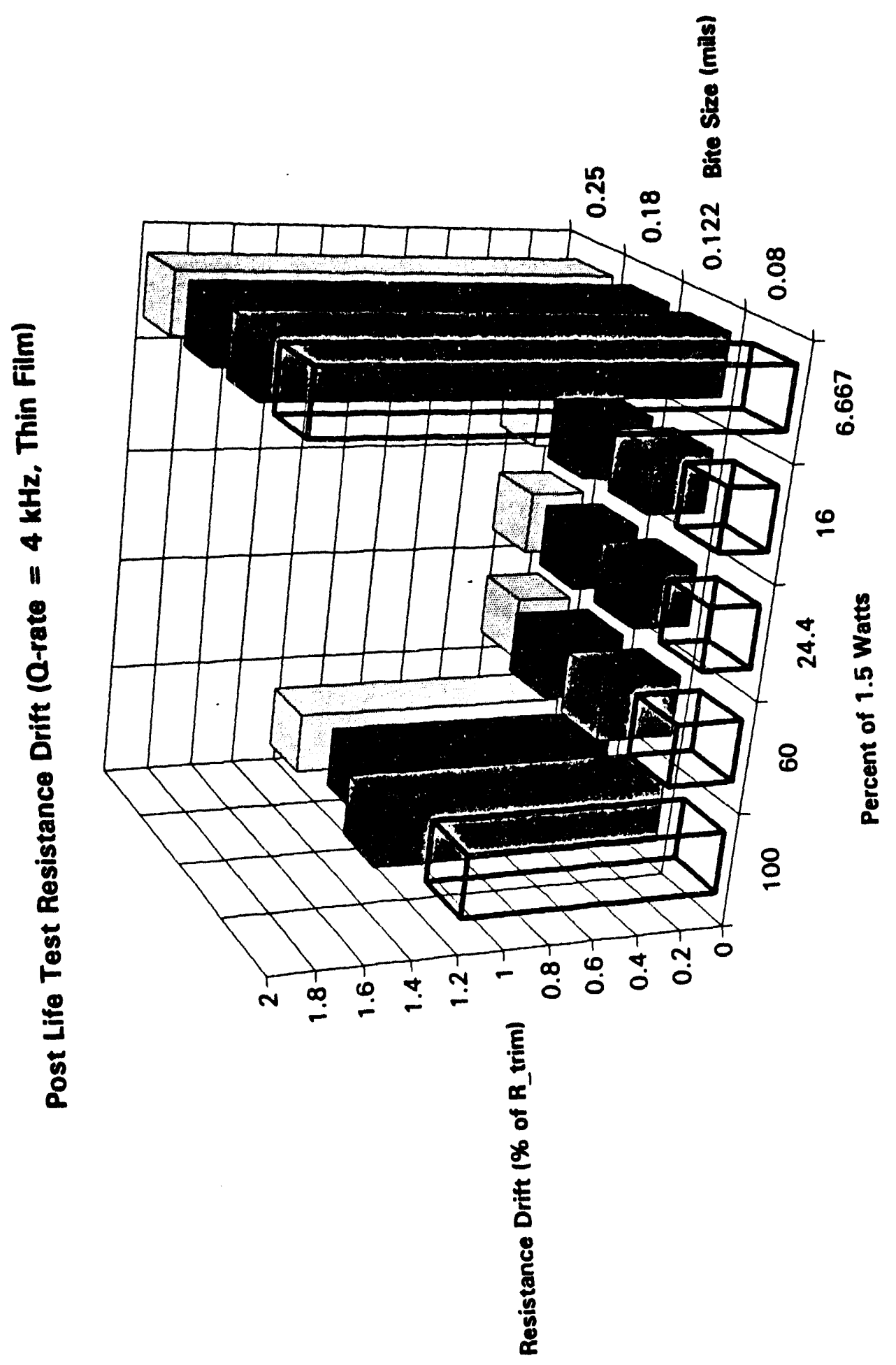


Histogram, nominal zup +20 mils (48 thin film resistors)

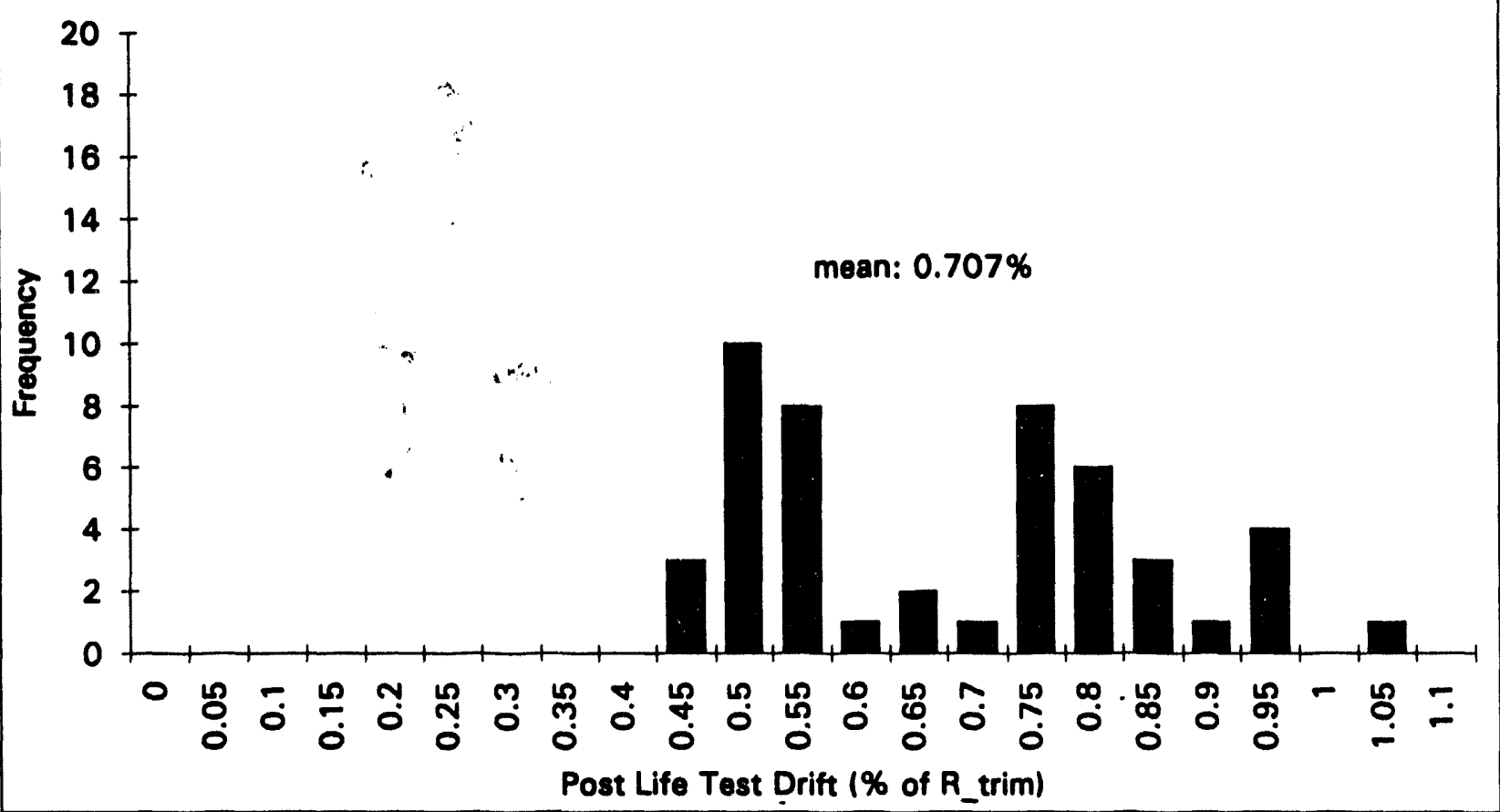

Histogram, nominal zup +0 mils (48 thin film resistors)

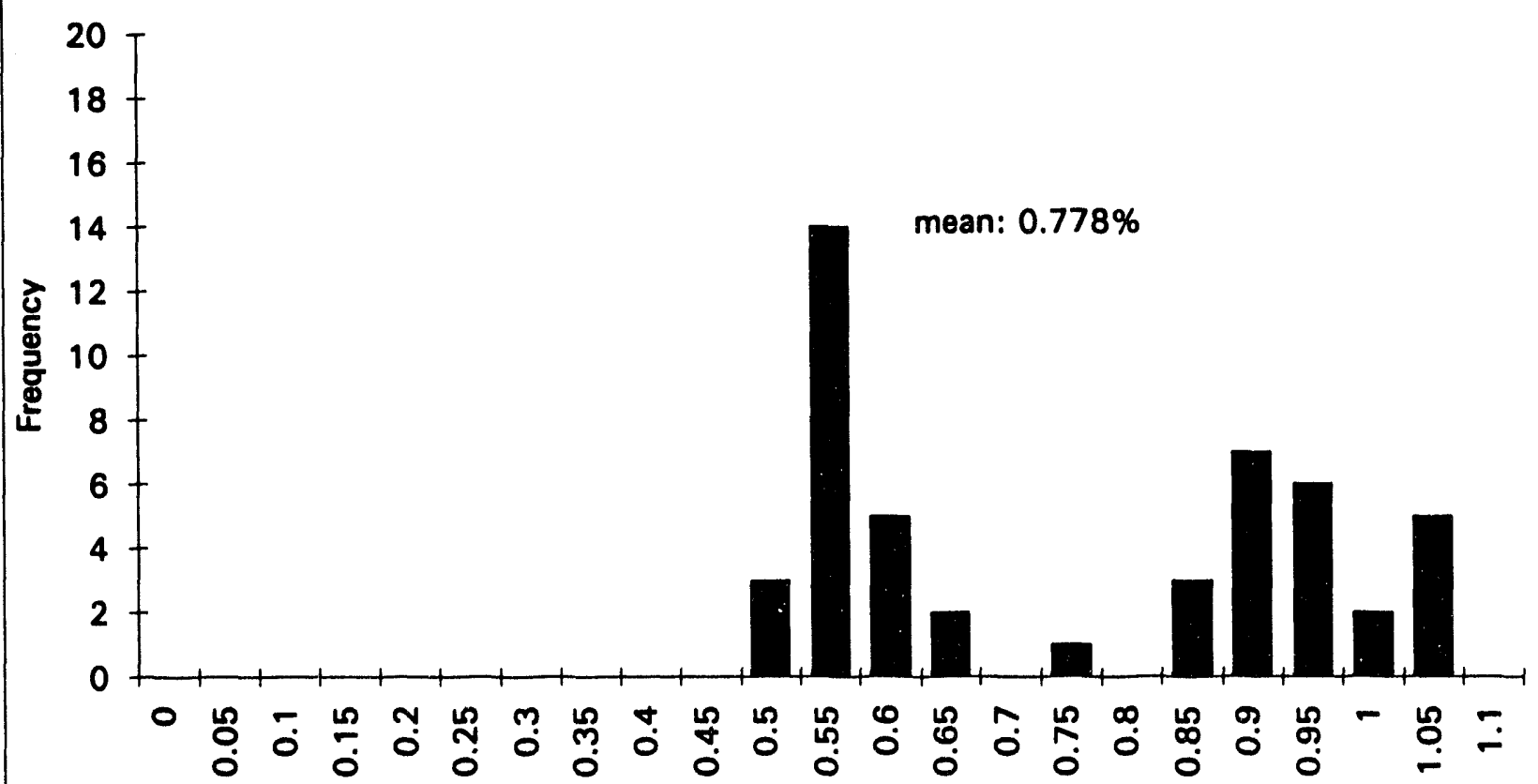

Post Life Test Drift (\% of R_trim) 
Histogram, nominal zup - 20 mils (48 thin film resistors)

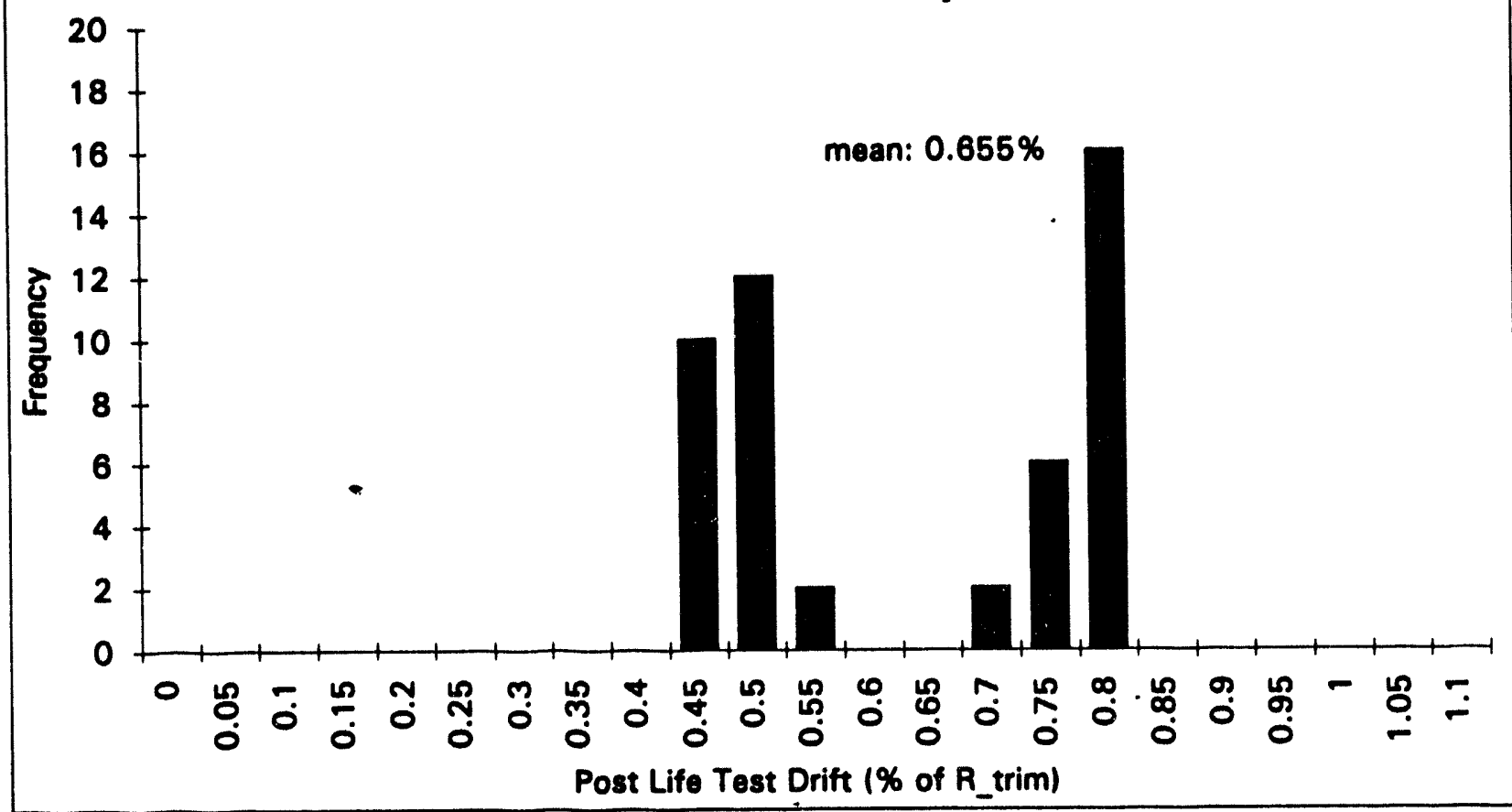

Histogram, nominal zup - 40 mils (48 thin film resistors)

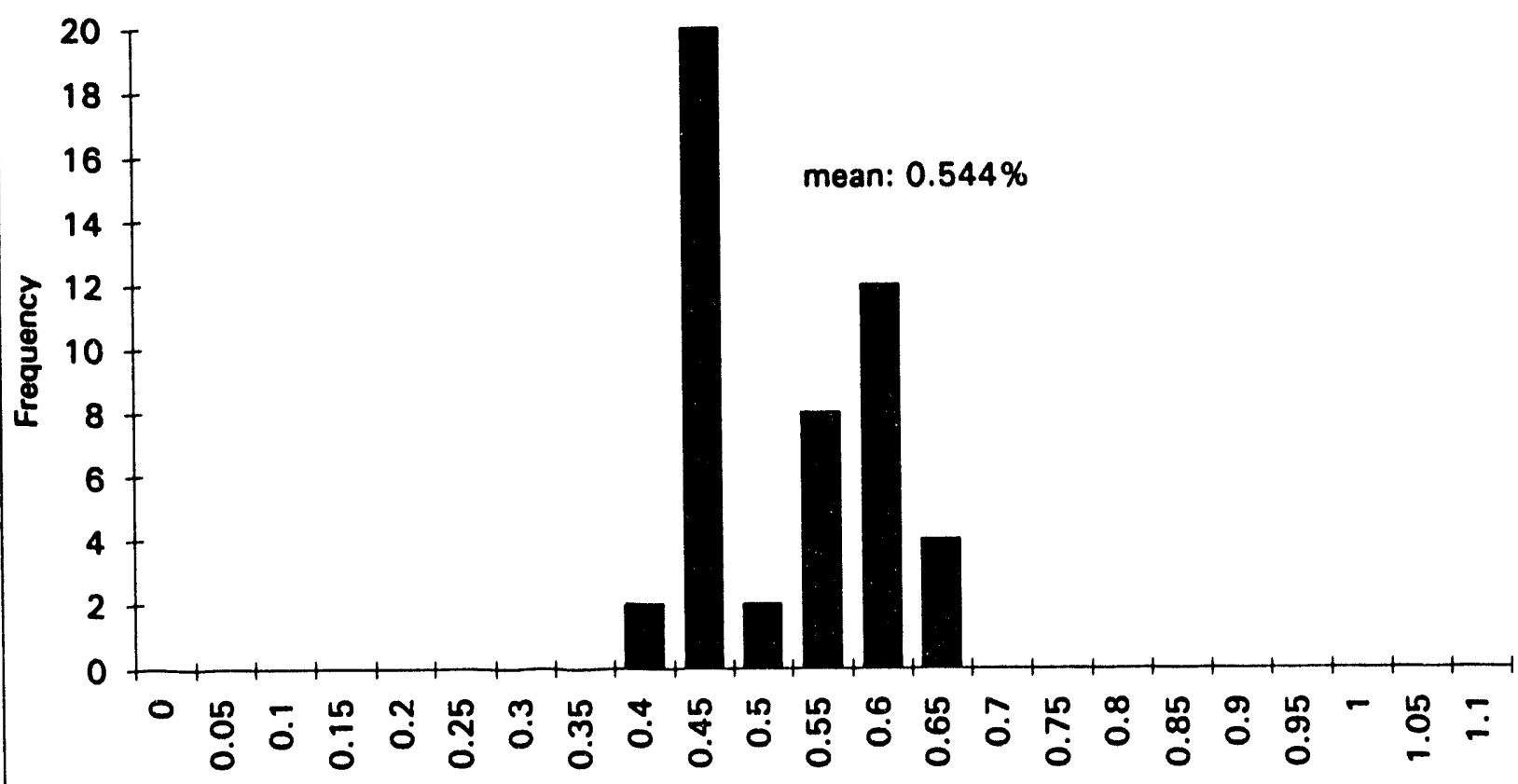

Post Life Test Drift (\% of R_trim) 

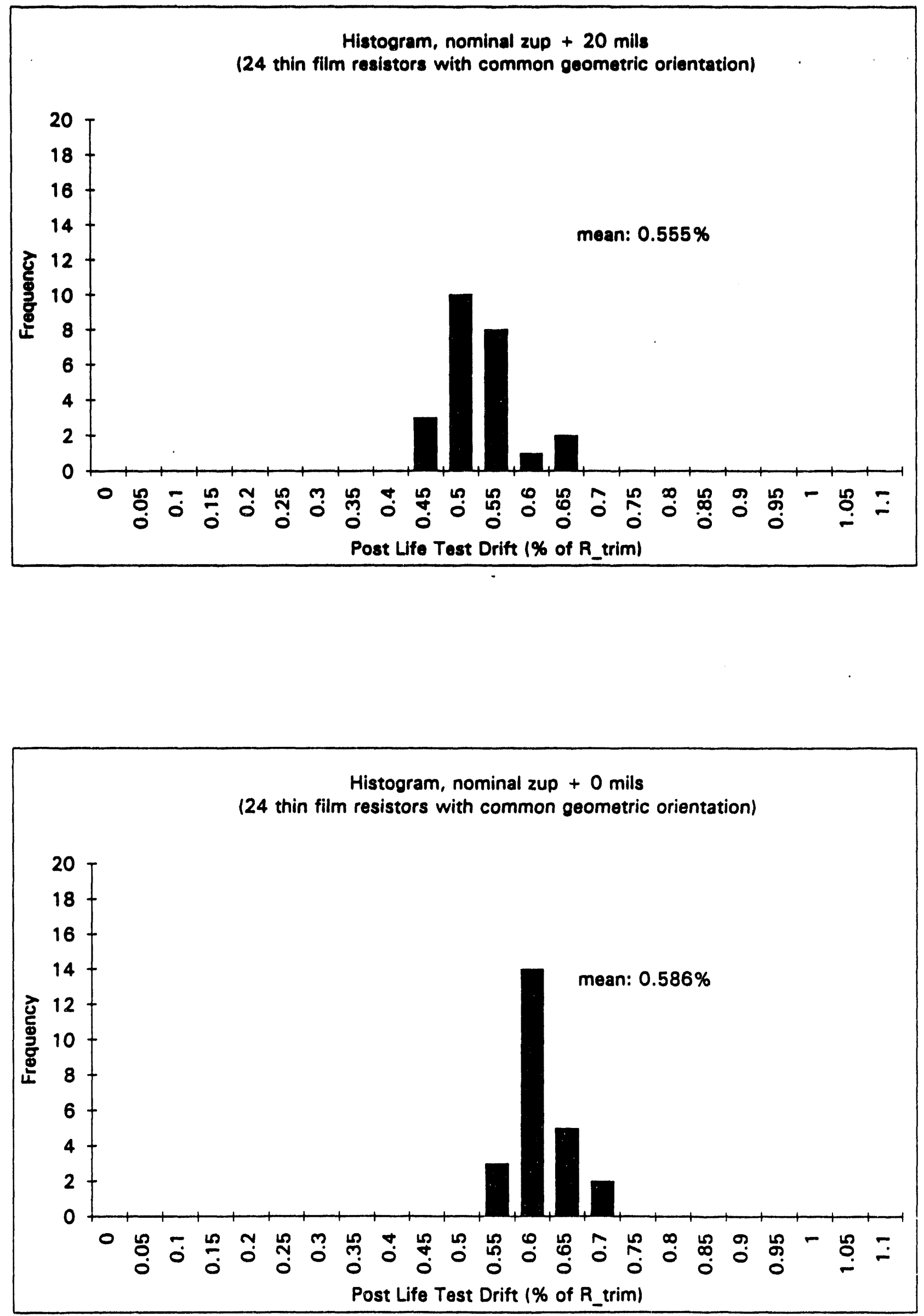
Histogram, nominal zup - 20 mils

(24 thin film resistors with common geometric orientation)

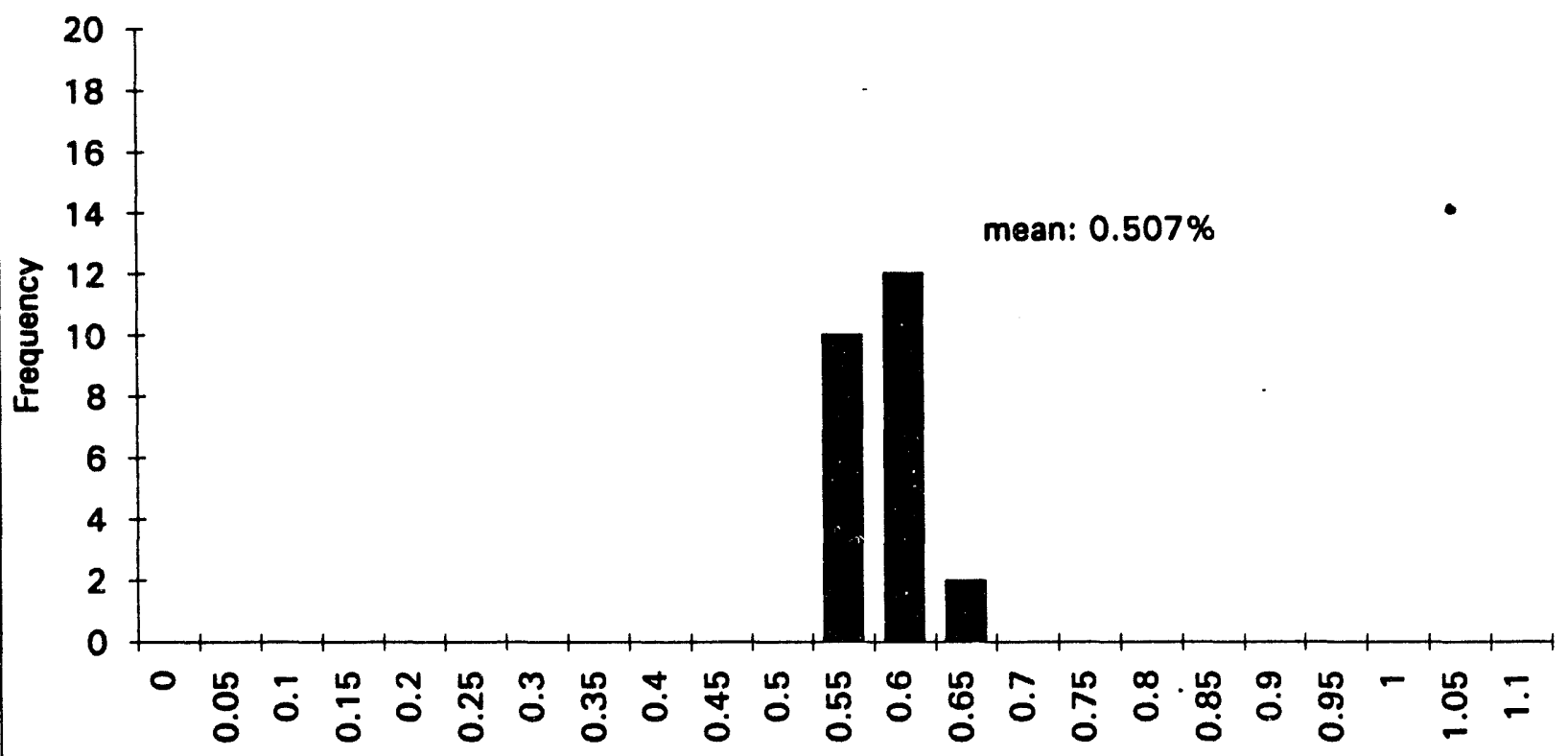

Post Life Test Drift (\% of R_trim)

Histogram, nominal zup - 40 mils

(24 thin film resistors with common geometric orientation)

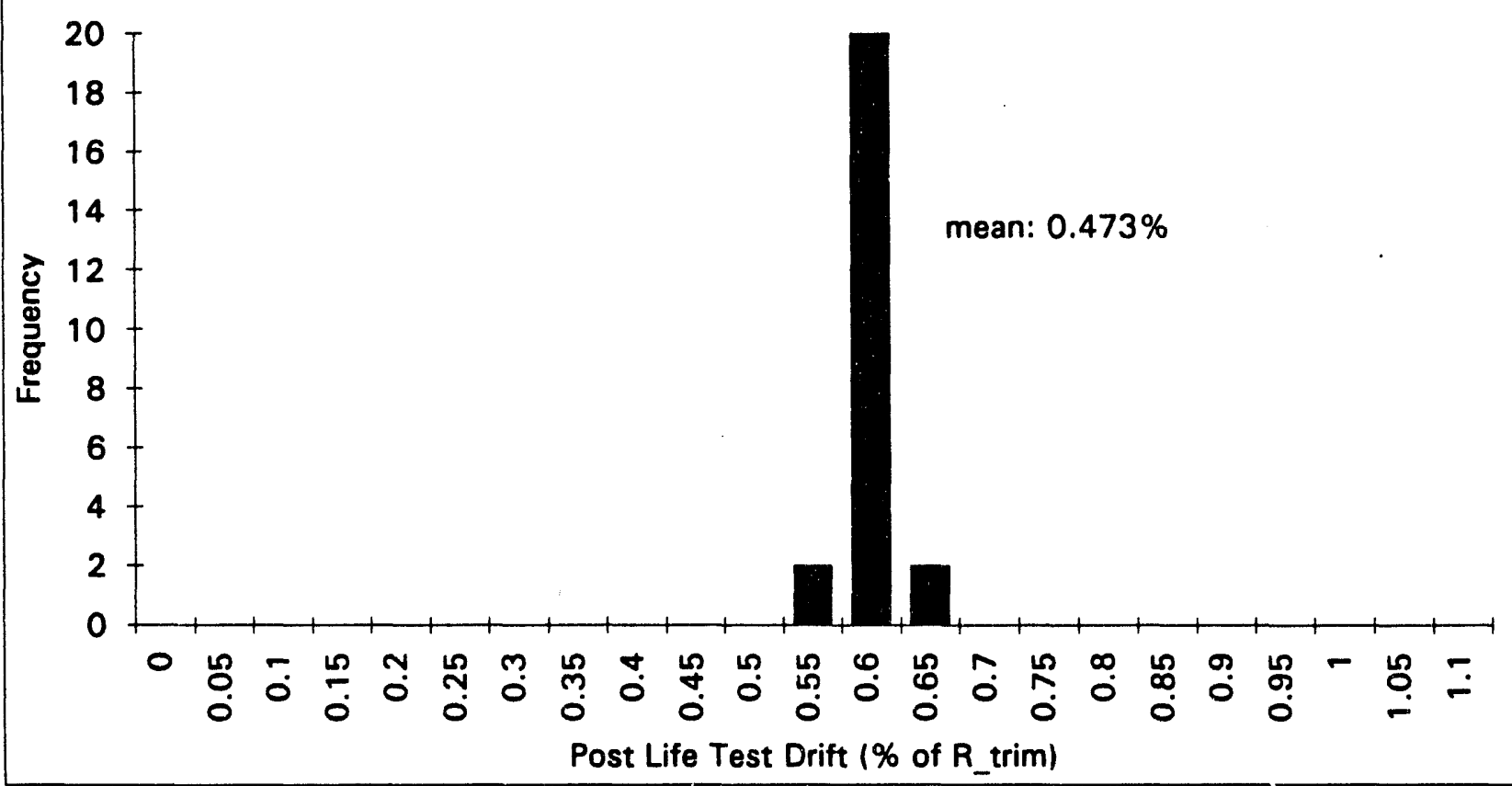


LASR0247.CSV

\begin{tabular}{|c|c|c|c|c|c|c|c|c|c|c|c|}
\hline HERMA & TYPE: & THIN FILN & & & & & & & & & \\
\hline HERMA & S/N: $\quad 1$ & 06 & & & & & & & & & \\
\hline RESIST & NCE PASTE & 100 & & & & & & & & & \\
\hline DATE: & $2 / 09 / 93$ & & & & & & & & & & \\
\hline TIME: & $4: 48: 30$ & & & & & & & & & & 20 \\
\hline EXPERII & IENT \#: 2 & & & & & & & & & & \\
\hline$R_{\text {nnum }}$ & R_trim & focus & R_24 & R_post_cyc & R_drift_cyc & R_post_life & R_drift_life & average & vert_avg & Bin & Frequency \\
\hline A1 & 4274.921 & 20 & 4282.87 & 4286.334 & 0.266976 & 4299.931 & 0.585032 & 0.70657 & \begin{tabular}{|l|}
0.55483 \\
\end{tabular} & 0 & 0 \\
\hline A2 & 4057.338 & 20 & 4064.08 & 4066.822 & 0.233749 & 4077.488 & 0.49664 & & & 0.05 & 0 \\
\hline A3 & 4120.573 & 20 & 4128.621 & 4132.102 & 0.279791 & 4145.094 & 0.595098 & & horiz avg & 0.1 & 0 \\
\hline A4 & 4237.94 & 20 & 4249.098 & 4254.378 & 0.387877 & 4273.197 & 0.831947 & & \begin{tabular}{|l|}
0.8583 \\
\end{tabular} & 0.15 & 0 \\
\hline A5 & 4167.624 & 20 & 4178.67 & 4183.68 & 0.385255 & 4201.749 & 0.818821 & & & 0.2 & 0 \\
\hline A6 & 4317.595 & 20 & 4331.121 & 4337.298 & 0.456342 & 4359.891 & 0.97963 & & & 0.25 & 0 \\
\hline A7 & 3922.216 & 20 & 3929.713 & 3932.597 & 0.264672 & 3943.842 & 0.551379 & & & 0.3 & 0 \\
\hline A8 & 3904.335 & 20 & 3911.141 & 3913.992 & 0.24734 & 3924.52 & 0.516998 & & & 0.35 & 0 \\
\hline A9 & 4003.142 & 20 & 4010.496 & 4013.481 & 0.258272 & 4024.881 & 0.543055 & & & 0.4 & 0 \\
\hline A10 & 4320.164 & 20 & 4333.62 & 4339.591 & 0.449682 & 4362.605 & 0.982382 & & & 0.45 & 3 \\
\hline A11 & 4191.012 & 20 & 4202.248 & 4207.214 & 0.386589 & 4225.327 & 0.818783 & & & 0.5 & 10 \\
\hline A12 & 4240.815 & 20 & 4252.087 & 4257.23 & 0.387072 & 4276.149 & 0.833194 & & & 0.55 & 8 \\
\hline B1 & 3869.654 & 20 & 3876.09 & 3878.817 & 0.236791 & 3888.726 & 0.492872 & & & 0.6 & 1 \\
\hline B2 & 3837.057 & 20 & 3843.494 & 3846.11 & 0.235936 & 3856.237 & 0.499853 & & & 0.65 & 2 \\
\hline B3 & 3747.548 & 20 & 3754.343 & 3756.973 & 0.251498 & 3767.35 & 0.528392 & & & 0.7 & 1 \\
\hline B4 & 3941.528 & 20 & 3952.174 & 3956.407 & 0.377493 & 3972.912 & 0.796237 & & & 0.75 & 8 \\
\hline B5 & 3987.278 & 20 & 3997.902 & 4002.301 & 0.376773 & 4019.077 & 0.797518 & & & 0.8 & 6 \\
\hline B6 & 4190.709 & 20 & 4203.235 & 4208.525 & 0.425131 & 4228.602 & 0.90421 & & & 0.85 & 3 \\
\hline B7 & 3838.18 & 20 & 3845.357 & 3848.367 & 0.265412 & 3859.437 & 0.553831 & & & 0.9 & 1 \\
\hline$B 8$ & 3840.886 & 20 & 3847.607 & 3850.525 & 0.250958 & 3860.96 & 0.522632 & & & 0.95 & 4 \\
\hline B9 & 3930.262 & 20 & 3937.089 & 3940.107 & 0.250492 & 3950.925 & 0.525732 & & & 1 & 0 \\
\hline B10 & 4129.403 & 20 & 4140.716 & 4145.702 & 0.394706 & 4163.759 & 0.831986 & & & 1.05 & 1 \\
\hline B11 & 4039.041 & 20 & 4049.446 & 4054.008 & 0.370558 & 4070.718 & 0.784273 & & & 1.1 & 0 \\
\hline B12 & 3987.331 & 20 & 3997.571 & 4001.896 & 0.365282 & 4018.211 & 0.774441 & & & & \\
\hline C1 & 3876.605 & 20 & 3883.366 & 3886.246 & 0.248697 & 3896.298 & 0.507995 & & & & \\
\hline C2 & 3766.914 & 20 & 3773.401 & 3776.001 & 0.241232 & 3786.145 & 0.51052 & & & & \\
\hline C3 & 3715.806 & 20 & 3722.295 & 3724.837 & 0.243043 & 3734.789 & 0.510874 & & & & \\
\hline $\mathrm{C4}$ & 3877.595 & 20 & 3887.461 & 3891.585 & 0.360791 & 3907.605 & 0.773944 & & & & \\
\hline
\end{tabular}




\begin{tabular}{|c|c|c|c|c|c|c|c|c|c|c|c|}
\hline C5 & 3999.745 & 20 & 4010.143 & 4014.948 & 0.380099 & 4031.623 & 0.797011 & & & & \\
\hline C6 & 4283.85 & 20 & 4297.578 & 4303.972 & 0.469718 & 4326.606 & 0.998074 & & & & \\
\hline C7 & 3967.645 & 20 & 3975.477 & 3978.899 & 0.283644 & 3991.147 & 0.592337 & & & & \\
\hline C8 & 3930.054 & 20 & 3937.158 & 3940.186 & 0.257808 & 3951.644 & 0.549354 & & & & \\
\hline C9 & 4051.276 & 20 & 4058.913 & 4062.292 & 0.271914 & 4073.995 & 0.56079 & & & & \\
\hline $\mathrm{C} 10$ & 4185.503 & 20 & 4197.64 & 4203.082 & 0.419997 & 4222.34 & 0.880105 & & & & \\
\hline C11 & 4086.063 & 20 & 4097.432 & 4102.465 & 0.401413 & 4120.599 & 0.84521 & & & & \\
\hline C12 & 3974.35 & 20 & 3984.46 & 3988.839 & 0.364563 & 4004.117 & 0.748974 & & & & \\
\hline D1 & 4064.832 & 20 & 4072.295 & 4075.667 & 0.266555 & 4087.25 & 0.551508 & & & & \\
\hline D2 & 3956.221 & 20 & 3963.003 & 3966.121 & 0.250239 & 3976.926 & 0.523363 & & & & \\
\hline D3 & 4046.705 & 20 & 4054.989 & 4058.788 & 0.298589 & 4072.837 & 0.645757 & & & & \\
\hline D4 & 4234.132 & 20 & 4246.177 & $425 T .598$ & 0.412505 & 4272.165 & 0.898256 & & & & \\
\hline D5 & 4398.686 & 20 & 4412.313 & 4418.55 & 0.451589 & 4442.142 & 0.987942 & & & & \\
\hline D6 & 4692.086 & 20 & 4707.762 & 4715.333 & 0.495451 & 4742.943 & 1.083895 & & & & \\
\hline D7 & 4491.928 & 20 & 4501.767 & 4506.067 & 0.314765 & 4522.181 & 0.673507 & & & & \\
\hline D8 & 4533.649 & 20 & 4543.473 & 4548.062 & 0.317912 & 4564.45 & 0.679396 & & & & \\
\hline D9 & 4521.833 & 20 & 4530.693 & 4534.496 & 0.280041 & 4548.916 & 0.598949 & & & & \\
\hline D10 & 4537.885 & 20 & 4551.06 & 4556.966 & 0.420482 & 4578.152 & 0.887358 & & & & \\
\hline D11 & 4313.515 & 20 & 4324.764 & 4329.67 & 0.374521 & 4347.182 & 0.780496 & & & & \\
\hline D12 & 4185.156 & 20 & 4195.851 & 4200.43 & 0.364957 & 4217.156 & 0.764609 & & vert_avg & & \\
\hline E1 & 4054.304 & 0 & 4062.017 & 4065.31 & 0.271465 & 4078.295 & 0.591729 & 0.77768 & 0.58584 & Bin & Frequency \\
\hline E2 & 4076.266 & 0 & 4084.413 & 4087.869 & 0.284648 & 4101.098 & 0.60918 & & & 0 & 0 \\
\hline E3 & 4075.27 & 0 & 4083.87 & 4087.642 & 0.303587 & 4102.177 & 0.660245 & & horiz avg & 0.05 & 0 \\
\hline E4 & 4268.237 & 0 & 4282.018 & 4288.857 & 0.483103 & 4314.028 & 1.072834 & & 0.96952 & 0.1 & 0 \\
\hline E5 & 4304.068 & 0 & 4317.458 & 4324.543 & 0.475713 & 4350.048 & 1.068294 & & & 0.15 & 0 \\
\hline E6 & 4473.017 & 0 & 4487.437 & 4494.988 & 0.49119 & 4521.729 & 1.08902 & & & 0.2 & 0 \\
\hline E7 & 4520.364 & 0 & 4529.663 & 4533.988 & 0.301392 & 4551.043 & 0.67868 & & & 0.25 & 0 \\
\hline E8 & 4540.824 & 0 & 4549.995 & 4554.087 & 0.292084 & 4569.93 & 0.640974 & & & 0.3 & 0 \\
\hline E9 & 4596.128 & 0 & 4605.036 & 4608.953 & 0.279039 & 4624.53 & 0.617945 & & & 0.35 & 0 \\
\hline E10 & 4427.159 & 0 & 4441.294 & 4448.291 & 0.477326 & 4473.445 & 1.045496 & & & 0.4 & 0 \\
\hline E11 & 4264.219 & 0 & 4276.559 & 4282.637 & 0.43192 & 4304.804 & 0.951763 & & & 0.45 & 0 \\
\hline E12 & 4129.149 & 0 & 4141.117 & 4146.839 & 0.428418 & 4168.072 & 0.942647 & & & 0.5 & 3 \\
\hline F1 & 3865.849 & 0 & 3873.113 & 3876.196 & 0.267651 & 3888.166 & 0.577292 & & & 0.55 & 14 \\
\hline F2 & 3832.661 & 0 & 3839.729 & 3842.742 & 0.263029 & 3854.187 & 0.561649 & & & 0.6 & 5 \\
\hline F3 & 3896.096 & 0 & 3903.695 & 3907.124 & 0.283053 & 3919.717 & 0.606264 & & & 0.65 & 2 \\
\hline
\end{tabular}




\section{LASR0247.CSV}

\begin{tabular}{|c|c|c|c|c|c|c|c|c|c|c|c|}
\hline F4 & 3982.4 & 0 & 3994.726 & 4000.522 & 0.455052 & 4021.964 & 0.993474 & & & 0.7 & 0 \\
\hline F6 & 4159.344 & 0 & 4173.059 & 4179.833 & 0.492602 & 4204.233 & 1.079226 & & & 0.8 & 0 \\
\hline F7 & 4077.639 & 0 & 4085.754 & 4089.348 & 0.287151 & 4103.179 & 0.626344 & & & 0.85 & 3 \\
\hline F8 & 3971.606 & 0 & 3978.83 & 3981.926 & 0.259845 & 3994.66 & 0.580476 & & & 0.9 & 7 \\
\hline Fio & 4102.284 & 0 & 4115.316 & 4121.649 & 0.472054 & 4145.009 & 1.041493 & & & 1 & 2 \\
\hline F11 & 3998.973 & 0 & 4010.974 & 4016.723 & 0.443864 & 4038.01 & 0.976166 & & & 1.05 & 5 \\
\hline F12 & 3917.816 & 0 & 3929.334 & 3934.635 & 0.429295 & 3954.722 & 0.941997 & & & 1.1 & 0 \\
\hline G3 & 3831.235 & 0 & 3838.87 & 3841.865 & 0.277456 & 3854.043 & 0.595314 & & & & \\
\hline G4 & 3907.425 & 0 & 3918.906 & 3923.695 & 0.416387 & 3943.26 & 0.917112 & & & & \\
\hline G5 & 3900.059 & 0 & 3911.363 & 3916.187 & 0.413532 & 3936.248 & 0.927906 & & & & \\
\hline G6 & 4005.245 & 0 & 4017.438 & 4022.715 & 0.436178 & 4043.569 & 0.956839 & & & & \\
\hline G7 & 3924.96 & 0 & 3932.244 & 3935.288 & 0.263136 & 3947.274 & 0.568507 & & & & \\
\hline G8 & 3893.66 & 0 & 3900.853 & 3903.949 & 0.26425 & 3915.699 & 0.56603 & & & & \\
\hline $\mathrm{H} 1$ & 4177.637 & 0 & 4185.546 & 4188.768 & 0.266442 & 4201.216 & 0.564407 & & & & \\
\hline $\mathrm{H} 2$ & 4123.008 & 0 & 4130.97 & 4134.258 & 0.272859 & 4146.878 & 0.578943 & & & & \\
\hline $\mathrm{H3}$ & 4115.759 & 0 & 4123.524 & 4126.618 & 0.26384 & 4138.554 & 0.553845 & & & & \\
\hline $\mathrm{H} 4$ & 4185.509 & 0 & 4197.824 & 4203.362 & 0.426543 & 4224.778 & 0.938211 & & & & \\
\hline H5 & 4128.141 & 0 & 4140.619 & 4146.219 & 0.437921 & 4167.909 & 0.963346 & & & & \\
\hline $\mathrm{H} 6$ & 4175.981 & 0 & 4189.006 & 4194.937 & 0.453929 & 4217.663 & 0.998143 & & & & \\
\hline $\mathrm{H7}$ & 3936.91 & 0 & 3944.619 & 3947.268 & 0.2631 & 3958.888 & 0.558259 & & & & \\
\hline $\mathrm{HB}$ & 3936.05 & 0 & 3943.568 & 3946.392 & 0.262751 & 3957.597 & 0.547418 & & & & \\
\hline H9 & 3970.979 & 0 & 3978.514 & 3981.199 & 0.257367 & 3991.91 & 0.527087 & & & & \\
\hline $\mathrm{H} 10$ & 4008.662 & 0 & 4020.394 & 4025.431 & 0.418319 & 4044.5 & 0.894006 & & & & \\
\hline $\mathrm{H} 11$ & 3973.117 & 0 & 3983.573 & 3988.049 & 0.375826 & 4004.345 & 0.785981 & & & & \\
\hline $\mathrm{H} 12$ & 4127.264 & 0 & 4139.277 & 4144.693 & 0.422289 & 4164.873 & 0.911241 & & vert_avg & & \\
\hline 11 & 4323.652 & -20 & 4330.787 & & & 4345.671 & 0.509278 & \begin{tabular}{|l|}
0.65524 \\
\end{tabular} & 0.50734 & Bin & Frequency \\
\hline
\end{tabular}




\begin{tabular}{|c|c|c|c|c|c|c|c|c|}
\hline 13 & 4315.641 & -20 & 4322.69 & 4337.845 & 0.51449 & horiz_avg & 0.05 & 0 \\
\hline 14 & 4333.48 & -20 & 4344.445 & 4369.512 & 0.831471 & 0.80314 & 0.1 & 0 \\
\hline 15 & 4212.805 & -20 & 4222.852 & 4246.453 & 0.798704 & & 0.15 & 0 \\
\hline 16 & 4238.217 & -20 & 4248.692 & 4273.549 & 0.833649 & & 0.2 & 0 \\
\hline 17 & 4182.894 & -20 & 4189.924 & 4204.431 & 0.514893 & & 0.25 & 0 \\
\hline 18 & 4132.58 & -20 & 4139.24 & 4152.645 & 0.485538 & & 0.3 & 0 \\
\hline 19 & 4221.334 & -20 & 4228.073 & 4241.192 & 0.470431 & & 0.35 & 0 \\
\hline 110 & 4060.767 & -20 & 4069.806 & 4089.417 & 0.705535 & & 0.4 & 0 \\
\hline 111 & 4078.127 & -20 & 4087.222 & 4107.236 & 0.713773 & & 0.45 & 10 \\
\hline 112 & 4280.839 & -20 & 4291.006 & 4313.757 & 0.76896 & & 0.5 & 12 \\
\hline J1 & 4041.526 & -20 & 4048.29 & 4061.63 & 0.497446 & & 0.55 & 2 \\
\hline $\mathrm{J} 2$ & 4064.611 & -20 & 4071.279 & 4084.851 & 0.497959 & & 0.6 & 0 \\
\hline J3 & 4036.174 & -20 & 4042.963 & 4056.941 & 0.514514 & & 0.65 & 0 \\
\hline $\mathrm{J4}$ & 4077.606 & -20 & 4087.709 & 4111.589 & 0.833415 & & 0.7 & 2 \\
\hline $\mathrm{J5}$ & 4070.886 & -20 & 4080.806 & 4104.178 & 0.81781 & & 0.75 & 6 \\
\hline 56 & 4115.65 & -20 & 4125.88 & 4149.443 & 0.821073 & & 0.8 & 16 \\
\hline $\mathrm{J7}$ & 4095.965 & -20 & 4102.367 & 4115.931 & 0.487449 & & 0.85 & 0 \\
\hline $\mathrm{J8}$ & $4 C 47.318$ & -20 & 4053.751 & 4066.933 & 0.484641 & & 0.9 & 0 \\
\hline j9 & 4126.6 & -20 & 4133.203 & 4146.527 & 0.4829 & & 0.95 & 0 \\
\hline $\mathrm{J10}$ & 4018.303 & -20 & 4027.994 & 4049.219 & 0.769378 & & 1 & 0 \\
\hline $\mathrm{J11}$ & 4014.195 & -20 & 4023.889 & 4046.013 & 0.79265 & & 1.05 & 0 \\
\hline$J 12$ & 4039.46 & -20 & 4049.331 & 4071.123 & 0.783835 & & 1.1 & 0 \\
\hline K1 & 4043.281 & -20 & 4050.144 & 4063.949 & 0.511158 & & & \\
\hline K2 & 4053.447 & -20 & 4060.151 & 4073.697 & 0.499568 & & & \\
\hline K3 & 4037.719 & -20 & 4044.475 & 4058.231 & 0.508021 & & & \\
\hline K4 & 4086.307 & -20 & 4096.55 & 4119.59 & 0.814497 & & & \\
\hline K5 & 4134.761 & -20 & 4145.125 & 4168.299 & 0.81113 & & & \\
\hline K6 & 4204.01 & -20 & 4214.564 & 4238.351 & 0.816851 & & & \\
\hline K7 & 4190.426 & -20 & 4197.244 & 4211.513 & 0.503212 & & & \\
\hline K8 & 4157.226 & -20 & 4164.002 & 4177.622 & 0.490627 & & & \\
\hline K9 & 4180.804 & -20 & 4187.94 & 4202.257 & 0.513126 & & & \\
\hline K10 & 4136.776 & -20 & 4147.208 & 4170.773 & 0.821834 & & & \\
\hline K11 & 4080.739 & -20 & 4090.881 & 4114.555 & 0.828683 & & & \\
\hline K12 & 4080.088 & -20 & 4090.336 & 4113.492 & 0.818698 & & & \\
\hline LI & 4223.554 & -20 & 4230.932 & 4245.321 & 0.515361 & & & \\
\hline L2 & 4209.405 & -20 & 4216.256 & 4230.247 & 0.495118 & & & \\
\hline
\end{tabular}




\begin{tabular}{|c|c|c|c|c|c|c|c|c|c|c|}
\hline L3 & 4218.774 & -20 & 4225.868 & & 4240.612 & 0.517627 & & & & \\
\hline$L 4$ & 4315.879 & -20 & 4326.89 & & 4352.281 & 0.843435 & & & & \\
\hline L5 & 4413.73 & -20 & 4424.2 & & 4449.083 & 0.800984 & & & & \\
\hline L6 & 4552.583 & -20 & 4563.671 & & 4590.259 & 0.827575 & & & & \\
\hline L7 & 4693.076 & -20 & 4701.467 & & 4719.287 & 0.558495 & & & & \\
\hline L8 & 4678.911 & -20 & 4687.081 & & 4704.33 & 0.543259 & & & & \\
\hline L9 & 4692.669 & -20 & 4701.172 & & 4718.518 & 0.55083 & & & & \\
\hline L10 & 4527.451 & -20 & 4538.493 & & 4564.441 & 0.817025 & & & & \\
\hline L11 & 4383.049 & -20 & 4393.841 & 2 & 4418.34 & 0.805172 & & & & \\
\hline L12 & 4306.106 & -20 & 4316.837 & & 4340.525 & 0.799302 & & & & \\
\hline M1 & 4433.45 & -40 & 4440.144 & & 4454.49 & 0.474583 & 0.54352 & 0.47344 & $\operatorname{Bin}$ & Frequency \\
\hline$\overline{M 2}$ & 4546.412 & -40 & 4553.57 & & 4568.474 & 0.485268 & & & 0 & 0 \\
\hline M3 & 4580.694 & -40 & 4587.492 & & 4602.967 & 0.486234 & & & 0.05 & 0 \\
\hline M4 & 4670.051 & -40 & 4678.876 & & 4700.731 & 0.556954 & & 0.61361 & 0.1 & 0 \\
\hline M5 & 4785.954 & -40 & 4794.818 & & 4817.578 & 0.660767 & & & 0.15 & 0 \\
\hline M6 & 4952.18 & -40 & 4961.479 & & 4985.827 & 0.679447 & & & 0.2 & 0 \\
\hline M7 & 5146.403 & -40 & 5154.427 & & 5174.086 & 0.537919 & & & 0.25 & 0 \\
\hline M8 & 4982.86 & -40 & 4990.763 & & 5009.219 & 0.528987 & & & 0.3 & 0 \\
\hline M9 & 4902.826 & -40 & 4910.371 & & 4927.252 & 0.498193 & & & 0.35 & 0 \\
\hline M10 & 4801.937 & -40 & 4810.903 & & 4833.221 & 0.651485 & & & 0.4 & 2 \\
\hline M11 & 4599.7 & -40 & 4607.983 & & 4627.843 & 0.611848 & & & 0.45 & 20 \\
\hline M12 & 4487.089 & -40 & 4495.007 & & 4513.551 & 0.58974 & & & 0.5 & 2 \\
\hline N1 & 4230.695 & -40 & 4236.963 & & 4249.85 & 0.452758 & & & 0.55 & 8 \\
\hline N2 & 4298.101 & -40 & 4304.389 & & 4317.945 & 0.461698 & & & 0.6 & 12 \\
\hline N3 & 4303.863 & -40 & 4310.306 & & 4324.141 & 0.471151 & & & 0.65 & 4 \\
\hline N4 & 4358.916 & -40 & 4367.019 & & 4385.887 & 0.618756 & & & 0.7 & 0 \\
\hline N5 & 4378.229 & -40 & 4386.1 .57 & & 4404.734 & 0.605389 & & & 0.75 & 0 \\
\hline N6 & 4445.601 & -40 & 4453.92 & & 4473.229 & 0.621475 & & & 0.8 & 0 \\
\hline N7 & 4522.648 & -40 & 4529.567 & & 4544.588 & 0.485112 & & & 0.85 & 0 \\
\hline N8 & 4376.634 & -40 & 4383.163 & & 4397.286 & 0.47188 & & & 0.9 & 0 \\
\hline N9 & 4353.966 & -40 & 4360.538 & & 4373.806 & 0.455667 & & & 0.95 & 0 \\
\hline N10 & 4301.063 & -40 & 4308.771 & & 4326.336 & 0.587588 & & & 1 & 0 \\
\hline N11 & 4233.135 & -40 & 4240.981 & & 4258.428 & 0.597491 & & & 1.05 & 0 \\
\hline N12 & 4194.916 & -40 & 4202.314 & & 4218.603 & 0.564651 & & & 1.1 & 0 \\
\hline 01 & 4242.807 & -40 & 4249.243 & & 4262.039 & 0.453279 & & & & \\
\hline
\end{tabular}




\section{LASR0247.CSV}

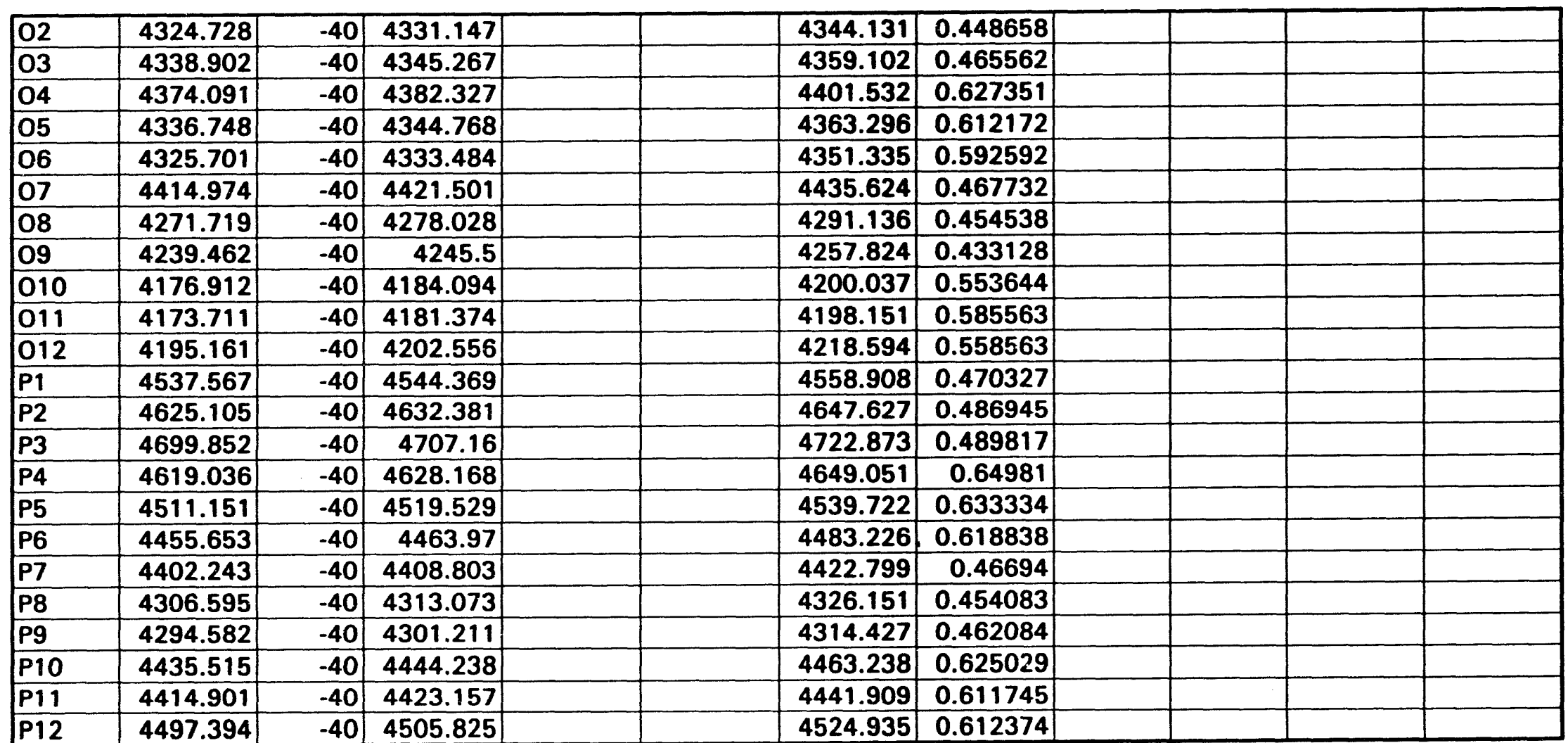



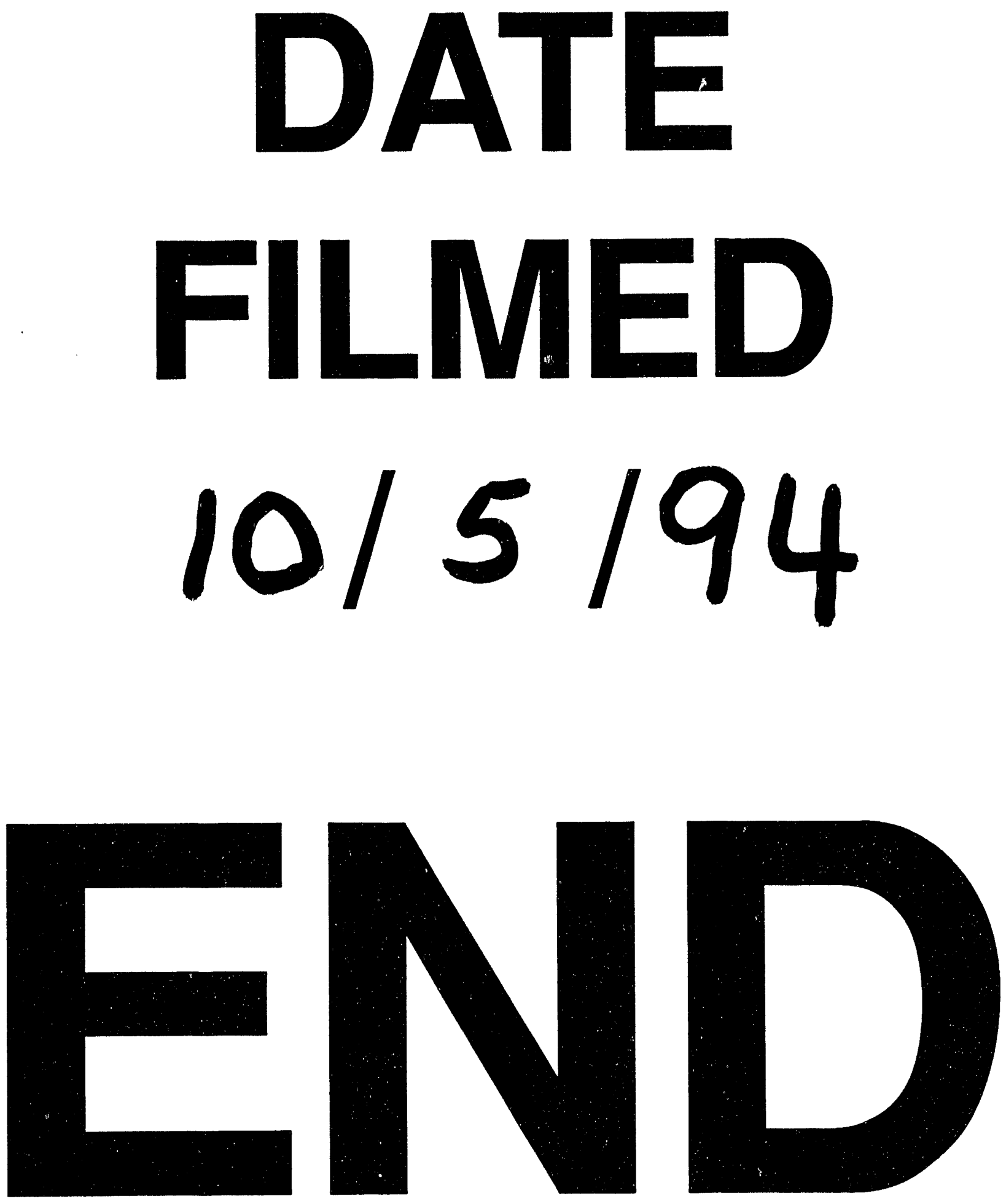
